\title{
Beginner's Luck
}

\section{A Language for Property-Based Generators}

\author{
Leonidas Lampropoulos ${ }^{1} \quad$ Diane Gallois-Wong ${ }^{2,3} \quad$ Cătălin $\mathrm{Hriţcu}^{2}$ \\ John Hughes ${ }^{4} \quad$ Benjamin C. Pierce ${ }^{1} \quad$ Li-yao Xia ${ }^{2,3}$ \\ ${ }^{1}$ University of Pennsylvania $\quad{ }^{2}$ INRIA Paris $\quad{ }^{3}$ ENS Paris $\quad{ }^{4}$ Chalmers University
}

\begin{abstract}
Property-based random testing à la QuickCheck requires building efficient generators for well-distributed random data satisfying complex logical predicates, but writing these generators can be difficult and error prone. We propose a domain-specific language in which generators are conveniently expressed by decorating predicates with lightweight annotations to control both the distribution of generated values and the amount of constraint solving that happens before each variable is instantiated. This language, called Luck, makes generators easier to write, read, and maintain.

We give Luck a formal semantics and prove several fundamental properties, including the soundness and completeness of random generation with respect to a standard predicate semantics. We evaluate Luck on common examples from the property-based testing literature and on two significant case studies, showing that it can be used in complex domains with comparable bug-finding effectiveness and a significant reduction in testing code size compared to handwritten generators.
\end{abstract}

\section{Introduction}

Since being popularized by QuickCheck [20], property-based random testing has become a standard technique for improving software quality in a wide variety of programming languages [2] 41, 48. 58] and for streamlining interaction with proof assistants [6. 16, 24, 57, 61].

When using a property-based random testing tool, one writes properties in the form of executable predicates. For example, a natural property to test for a list reverse function is that, for any list xs, reversing xs twice yields xs again. In QuickCheck notation:

prop_reverse $\mathrm{xs}=($ reverse $($ reverse $\mathrm{xs})==\mathrm{xs})$

To test this property, QuickCheck generates random lists until either it finds a counterexample or a predetermined number of tests succeed.

An appealing feature of QuickCheck is that it offers a library of property combinators resembling standard logical operators. For example, a property of the form $p==>$, built using the implication combinator $==>$, will be tested automatically by generating valuations (assignments of random values, of appropriate type, to the free variables of $p$ and $q$ ), discarding those valuations that fail to satisfy $p$, and checking whether any of the ones that remain are counterexamples to $\mathrm{q}$.

QuickCheck users soon learn that this default generate-andtest approach sometimes does not give satisfactory results. In particular, if the precondition $p$ is satisfied by relatively few values of the appropriate type, then most of the random inputs that QuickCheck generates will be discarded, so that q will seldom be exercised. Consider, for example, testing a simple prop- erty of a school database system: that every student in a list of registeredStudents should be taking at least one course,

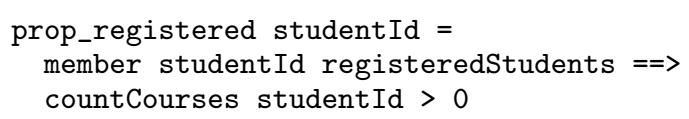

where, as usual:

member $\mathrm{x}[]=$ False

member $x(h: t)=(x==h) \|$ member $x t$

If the space of possible student ids is large (e.g., because they are represented as machine integers), then a randomly generated id is very unlikely to be a member of registeredStudents, so almost all test cases will be discarded.

To enable effective testing in such cases, the QuickCheck user can provide a property-based generator for inputs satisfying $\mathrm{p}$ here, a generator that always returns student ids drawn from the members of registeredStudents. Indeed, QuickCheck provides a library of combinators for defining such generators. These combinators also allow fine control over the distribution of generated values - a crucial feature in practice [20, 35 39].

Property-based generators generators work well for small to medium-sized examples, but writing them can become challenging as $\mathrm{p}$ gets more complex-sometimes turning into a research contribution in its own right! For example, papers have been written about random generation techniques for well-typed lambda-terms [25, 60, 64, 68] and for "indistinguishable" machine states that can be used for finding bugs in information-flow monitors [39, 40]. Moreover, if we use QuickCheck to test an invariant property (e.g., type preservation), then the same condition will appear in both the precondition and the conclusion of the property, requiring that we express this condition both as a boolean predicate $\mathrm{p}$ and as a generator whose outputs all satisfy $p$. These two artifacts must then be kept in sync, which can become both a maintenance issue and a rich source of confusion in the testing process. These difficulties are not hypothetical: Hriţcu et al.'s machine-state generator [39] is over 1500 lines of tricky Haskell, while Pałka et al.'s generator for welltyped lambda-terms [60] is over 1600 even trickier ones. To enable effective property-based random testing of complex software artifacts, we need a better way of writing predicates and corresponding generators.

A natural idea is to derive an efficient generator for a given predicate $\mathrm{p}$ directly from $\mathrm{p}$ itself. Indeed, two variants of this idea, with complementary strengths and weaknesses, have been explored by others - one based on local choices and backtracking, one on general constraint solving. Our language, Luck, synergistically combines these two approaches.

The first approach can be thought of as a kind of incremental generate-and-test: rather than generating completely random valu- 
ations and then testing them against $p$, we instead walk over the structure of $p$ and instantiate each unknown variable $x$ at the first point where we meet a constraint involving $\mathrm{x}$. In the member example above, on each recursive call, we make a random choice between the branches of the II. If we choose the left, we instantiate $x$ to the head of the list; otherwise we leave $\mathrm{x}$ unknown and continue with the recursive call to member on the tail. This has the effect of traversing the list of registered students and picking one of its elements. This process resembles narrowing from functional logic programming [1, 37, 48, 69]. It is attractively lightweight, admits natural control over distributions (as we will see in the next section), and has been used successfully [17, 26, 29, 63], even in challenging domains such as generating well-typed programs to test compilers [18, 25].

However, choosing a value for an unknown when we encounter the first constraint on it risks making choices that do not satisfy later constraints, forcing us to backtrack and make a different choice when the problem is discovered. For example, consider the notMember predicate:

$$
\begin{array}{ll}
\text { notMember } x[] & =\text { True } \\
\text { notMember } x(h: t) & =(x /=h) \text { \&\& notMember } x t
\end{array}
$$

Suppose we wish to generate values for $\mathrm{x}$ such that notMember $\mathrm{x}$ ys for some predetermined list ys. When we first encounter the constraint $\mathrm{x} /=\mathrm{h}$, we generate a value for $\mathrm{x}$ that is not equal to the known value $\mathrm{h}$. We then proceed to the recursive call of notMember, where we check that the chosen $\mathrm{x}$ does not appear in the rest of the list. Since the values in the rest of the list are not taken into account when choosing $\mathrm{x}$, this may force us to backtrack if our choice of $x$ was unlucky. If the space of possible values for $x$ is not much bigger than the length of ys — say, just twice as big - then we will backtrack $50 \%$ of the time. Worse yet, if notMember is used to define another predicate-e.g., distinct, which tests whether each element of an input list is different from all the others-and we want to generate a list satisfying distinct, then notMember's $50 \%$ chance of backtracking will be compounded on each recursive call of distinct, leading to unacceptably low rates of successful generation.

The second existing approach uses a constraint solver to generate a diverse set of valuations satisfying a predicate ${ }^{1}$ This approach has been widely investigated, both for generating inputs directly from predicates [13, 34, 46, 66] and for symbolic-executionbased testing [3, 9, 30, 67, 70], which additionally uses the system under test to guide generation of inputs that exercise different control-flow paths. For notMember, gathering a set of disequality constraints on $\mathrm{x}$ before choosing its value avoids any backtracking.

However, pure constraint-solving approaches do not give us everything we need. They do not provide effective control over the distribution of generated valuations. At best, they might guarantee a uniform (or near uniform) distribution [15], but this is typically not the distribution we want in practice (see \$2). Moreover, the overhead of maintaining and solving constraints can make these approaches significantly less efficient than the more lightweight, local approach of needed narrowing when the latter does not lead to backtracking, as for instance in member.

The complementary strengths and weaknesses of local instantiation and global constraint solving suggest a hybrid approach, where limited constraint propagation, under explicit user control, is used to refine the domains (sets of possible values) of unknowns before

${ }^{1}$ Constraint solvers can, of course, be used to directly search for counterexamples to a property of interest by software model checking [4, 5, 42, 44. etc.]. We are interested here in the rather different task of quickly generating a large number of diverse inputs, so that we can thoroughly test systems like compilers whose state spaces are too large to be exhaustively explored. instantiation. Exploring this approach is the goal of this paper. Our main contributions are:

- We propose a new domain-specific language, Luck, for writing generators via lightweight annotations on predicates, combining the strengths of the local-instantiation and constraintsolving approaches to generation. Section \$2 illustrates Luck's novel features using binary search trees as an example.

- To place Luck's design on a firm formal foundation, we define a core calculus and establish key properties, including the soundness and completeness of its probabilistic generator semantics with respect to a straightforward interpretation of expressions as predicates $\$ 3$.

- We provide a prototype interpreter $[\$ 4]$ including a simple implementation of the constraint-solving primitives used by the generator semantics. We do not use an off-the shelf constraint solver because we want to experiment with a per-variable uniform sampling approach $\$ 2$ which is not supported by modern solvers. In addition, using such a solver would require translating Luck expressions-datatypes, pattern matching, etc.-into a form that it can handle. We leave this for future work.

- We evaluate Luck's expressiveness on a collection of common examples from the random testing literature $(\$ 5)$ and on two significant case studies; the latter demonstrate that Luck can be used (1) to find bugs in a widely used compiler (GHC) by randomly generating well-typed lambda terms and (2) to help design information-flow abstract machines by generating "lowindistinguishable" machine states. Compared to hand-written generators, these experiments show comparable bug-finding effectiveness (measured in test cases generated per counterexample found) and a significant reduction in the size of testing code. The interpreted Luck generators run an order of magnitude slower than compiled QuickCheck versions ( 8 to 24 times per test), but many opportunities for optimization remain.

Sections \$6 and $\$ 7$ discuss related work and future directions. This paper is accompanied by several auxiliary materials: (1) a Coq formalization of the narrowing semantics of Luck and machinechecked proofs of its properties (available at https://github. com/QuickChick/Luck \$3.3; (2) the prototype Luck interpreter and a battery of example programs, including all the ones we used for evaluation (also at https://github.com/QuickChick/ Luck) \$5; (3) an extended version of the paper with full definitions and paper proofs for the whole semantics (https://arxiv. org/abs/1607.05443).

\section{Luck by Example}

Fig. 1 shows a recursive Haskell predicate bst that checks whether a given tree with labels strictly between low and high satisfies the standard binary-search tree (BST) invariant [56]. It is followed by a QuickCheck generator genTree, which generates BSTs with a given maximum depth, controlled by the size parameter. This generator first checks whether low $+1>=$ high, in which case it returns the only valid BST satisfying this constraint-the Empty one. Otherwise, it uses QuickCheck's frequency combinator, which takes a list of pairs of positive integer weights and associated generators and randomly selects one of the generators using the probabilities specified by the weights. In this example, $\frac{1}{\text { size }+1}$ of the time it creates an Empty tree, while $\frac{s i z e}{\text { size }+1}$ of the time it returns a Node. The Node generator is specified using monadic syntax: first it generates an integer $\mathrm{x}$ that is strictly between low and high, and then the left and right subtrees $I$ and $r$ by calling genTree recursively; finally it returns Node $x 1 \mathrm{r}$. 
Binary tree datatype (in both Haskell and Luck):

data Tree a = Empty $\mid$ Node a (Tree a) (Tree a)

Test predicate for BSTs (in Haskell):

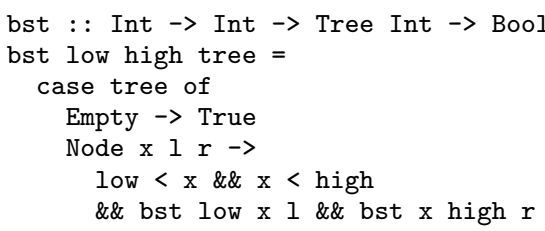

QuickCheck generator for BSTs (in Haskell):

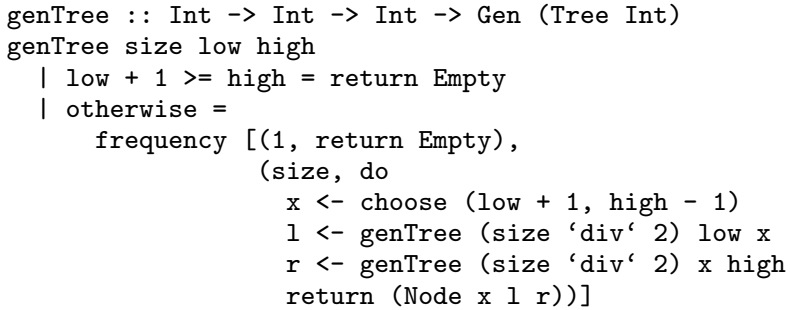

Luck generator (and predicate) for BSTs:

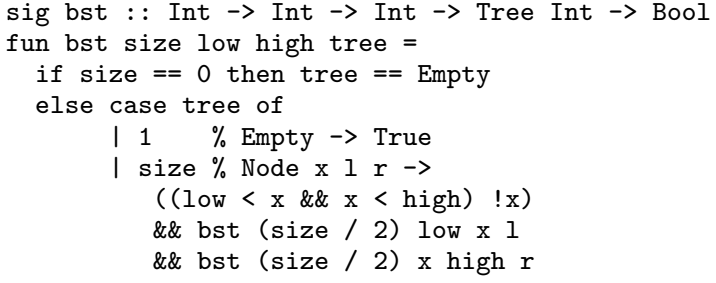

Figure 1. Binary Search Tree tester and two generators

The generator for BSTs allows us to efficiently test conditional properties of the form "if bst $t$ then $\langle$ some other property of $t\rangle$," but it raises some new issues of its own. First, even for this simple example, getting the generator right is a bit tricky (for instance because of potential off-by-one errors in generating $\mathrm{x}$ ), and it is not immediately obvious that the set of trees generated by the generator is exactly the set accepted by the predicate. Worse, we now need to maintain two similar but distinct artifacts and keep them in sync. (We can't just throw away the predicate and keep the generator because we often need them both, for example to test properties like "the insert function applied to a BST and a value returns a BST.") As predicates and generators become more complex, these issues can become quite problematic (e.g., [39]).

Enter Luck. The bottom of Fig. 1 shows a Luck program that represents both a BST predicate and a generator for random BSTs. Modulo variations in concrete syntax, the Luck code follows the Haskell bst predicate quite closely. The significant differences are: (1) the sample-after expression ! $\mathrm{x}$, which controls when node labels are generated, and (2) the size parameter, which is used, as in the QuickCheck generator, to annotate the branches of the case with relative weights. Together, these enable us to give the program both a natural interpretation as a predicate (by simply ignoring weights and sampling expressions) and an efficient interpretation as a generator of random trees with the same distribution as the QuickCheck version. For example, evaluating the top-level query bst $10042 \mathrm{u}=$ True-i.e., "generate values $t$ for the unknown u such that bst 10042 t evaluates to True"-will yield random binary search trees of size up to 10 with node la- bels strictly between 0 and 42 , with the same distribution as the QuickCheck generator genTree $10 \quad 042$.

An unknown in Luck is a special kind of value, similar to logic variables found in logic programming languages and unification variables used by type-inference algorithms. Unknowns are typed, and each is associated with a domain of possible values from its type. Given an expression $e$ mentioning some set $U$ of unknowns, our goal is to generate valuations over these unknowns (maps from $U$ to concrete values) by iteratively refining the unknowns' domains, so that, when any of these valuations is substituted into $e$, the resulting concrete term evaluates to a desired value (e.g., True).

Unknowns can be introduced both explicitly, as in the toplevel query above (see also \$4, and implicitly, as in the generator semantics of case expressions. In the bst example, when the Node branch is chosen, the pattern variables $x, 1$, and $r$ are replaced by fresh unknowns, which are then instantiated by evaluating the constraint low $<\mathrm{x} \& \& \mathrm{x}<$ high and the recursive calls to bst.

Varying the placement of unknowns in the top-level bst query yields different behaviors. For instance, if we change the query to bst $10 \mathrm{ul}$ uh $\mathrm{u}=$ True, replacing the low and high parameters with unknowns ul and uh, the domains of these unknowns will be refined during tree generation and the result will be a generator for random valuations $(\mathrm{ul} \mapsto \mathrm{i}, \mathrm{uh} \mapsto \mathrm{j}, \mathrm{u} \mapsto \mathrm{t})$ where $i$ and $\mathrm{j}$ are lower and upper bounds on the node labels in $t$.

Alternatively, we can evaluate the top-level query bst 100 $42 \mathrm{t}=$ True, replacing $\mathrm{u}$ with a concrete tree $\mathrm{t}$. In this case, Luck will return a trivial valuation only if $t$ is a binary search tree; otherwise it will report that the query is unsatisfiable. A less useful possibility is that we provide explicit values for low and high but choose them with low > high, e.g., bst $1064 \mathrm{u}$ $=$ True. Since there are no satisfying valuations for $u$ other than Empty, Luck will now generate only Empty trees.

A sample-after expression of the form e $\mathrm{m}$ is used to control instantiation of unknowns. Typically, $x$ will be an unknown $u$, and evaluating e $\mathrm{l} u$ will cause $u$ to be instantiated to a concrete value (after evaluating e to refine the domains of all of the unknowns in e). If $x$ reduces to a value rather than an unknown, we similarly instantiate any unknowns appearing within this value.

As a concrete example, consider the compound inequality constraint $0<\mathrm{x}$ \&\& $\mathrm{x}<4$. A generator based on pure narrowing (as in [29]), would instantiate $x$ when the evaluator meets the first constraint where it appears, namely $0<\mathrm{x}$ (assuming left-to-right evaluation order). We can mimic this behavior in Luck by writing ( 0 $<\mathrm{x})$ ! $\mathrm{x})$ \&\& $(\mathrm{x}<4)$. However, picking a value for $\mathrm{x}$ at this point ignores the constraint $\mathrm{x}<4$, which can lead to backtracking. If, for instance, the domain from which we are choosing values for $\mathrm{x}$ is 32-bit integers, then the probability that a random choice satisfying $0<\mathrm{x}$ will also satisfy $\mathrm{x}<4$ is minuscule. It is better in this case to write $(0<\mathrm{x} \& \& \mathrm{x}<4)$ ! $\mathrm{x}$, instantiating $\mathrm{x}$ after the entire conjunction has been evaluated and all the constraints on the domain of $\mathrm{x}$ recorded and thus avoiding backtracking completely. Finally, if we do not include a sample-after expression for $\mathrm{x}$ here at all, we can further refine its domain with constraints later on, at the cost of dealing with a more abstract representation of it internally in the meantime. Thus, sample-after expressions give Luck users explicit control over the tradeoff between the expense of possible backtracking — when unknowns are instantiated early - and the expense of maintaining constraints on unknowns-so that they can be instantiated late (e.g., so that $\mathrm{x}$ can be instantiated after the recursive calls to bst).

Sample-after expressions choose random values with uniform probability from the domain associated with each unknown. While this behavior is sometimes useful, effective property-based random testing often requires fine control over the distribution of generated test cases. Drawing inspiration from the QuickCheck com- 
binator library for building complex generators, and particularly frequency (which we saw in genTree (Fig. 1), Luck also allows weight annotations on the branches of a case expression which have a frequency-like effect. In the Luck version of bst, for example, the unknown tree is either instantiated to an Empty tree $\frac{1}{1+\text { size }}$ of the time or partially instantiated to a Node (with fresh unknowns for $\mathrm{x}$ and the left and right subtrees) $\frac{\text { size }}{1+\text { size }}$ of the time.

Weight annotations give the user control over the probabilities of local choices. These do not necessarily correspond to a specific posterior probability, but the QuickCheck community has established techniques for guiding the user in tuning local weights to obtain good testing. For example, the user can wrap properties inside a collect x combinator; during testing, QuickCheck will gather information on $\mathrm{x}$, grouping equal values to provide an estimate of the posterior distribution that is being sampled. The collect combinator is an effective tool for adjusting frequency weights and dramatically increasing bug-finding rates (e.g., [39]). The Luck implementation provides a similar primitive.

One further remark on uniform sampling: While locally instantiating unknowns uniformly from their domain is a useful default, generating globally uniform distributions of test cases is usually not what we want, as this often leads to inefficient testing in practice. A simple example comes from the information flow control experiments of Hriţcu et al. [39]. There are two "security levels," called labels, Low and High, and pairs of integers and labels are considered "indistinguishable" to a Low observer if the labels are equal and, if the labels are Low, so are the integers. In Haskell:

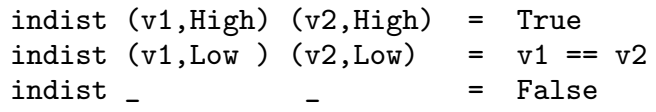

If we use 32-bit integers, then for every Low indistinguishable pair there are $2^{32}$ High ones! Thus, choosing a uniform distribution over indistinguishable pairs means that we will essentially never generate pairs with Low labels. Clearly, such a distribution cannot provide effective testing; indeed, Hriţcu et al. found that the best distribution was actually somewhat skewed in favor of Low labels.

However, in other areas where random sampling is used, it is sometimes important to be able to generate globally uniform distributions; if desired, this effect can be achieved in Luck by emulating Boltzmann samplers [22]. This technique fits naturally in Luck, providing an efficient way of drawing samples from combinatorial structures of approximate size $\mathrm{n}$-in time linear in $\mathrm{n}$ - where any two objects with the same size have an equal probability of being generated. Details can be found in Section 6

\section{Semantics of Core Luck}

We next present a core calculus for Luck-a minimal subset into which the examples in the previous section can in principle be desugared (though our implementation does not do this). The core omits primitive booleans and integers and replaces datatypes with binary sums, products, and iso-recursive types.

We begin in $\$ 3.1$ with the syntax and standard predicate semantics of the core. (We call it the "predicate" semantics because, in our examples, the result of evaluating a top-level expression will typically be a boolean, though this expectation is not baked into the formalism.) We then build up to the full generator semantics in three steps. First, we give an interface to a constraint solver $\$ 3.2$, abstracting over the primitives required to implement our semantics. Then we define a probabilistic narrowing semantics, which enhances the local-instantiation approach to random generation with QuickCheck-style distribution control \$3.3. Finally, we introduce a matching semantics, building on the narrowing semantics, that unifies constraint solving and narrowing into a single

$$
\begin{aligned}
v::= & ()|(v, v)| L_{T} v \mid R_{T} v \\
\mid & \operatorname{rec}\left(f: T_{1} \rightarrow T_{2}\right) x=e \mid \text { fold }_{T} v \\
& \mid u \\
e::= & x|()| \operatorname{rec}\left(f: T_{1} \rightarrow T_{2}\right) x=e \mid(e e) \\
\mid & (e, e) \mid \text { case e of }(x, y) \rightarrow e \\
\mid & L_{T} e \mid R_{T} \text { e } \mid \text { case e of }(L x \rightarrow e)(R x \rightarrow e) \\
\mid & f_{T} e l \text { unfold }_{T} \text { e } \\
\mid & u|e \leftarrow(e, e)| ! e \mid e: e \\
\bar{T}::= & X|1| \bar{T}+\bar{T}|\bar{T} \times \bar{T}| \mu X . \bar{T} \\
T::= & X|1| T+T|T \times T| \mu X . T \mid T \rightarrow T \\
\Gamma::= & \varnothing \mid \Gamma, x: T
\end{aligned}
$$

Figure 2. Core Luck Syntax

evaluator \$3.4. We also show how integers and booleans can be encoded and how the semantics applies to the binary search tree example \$3.5. The key properties of the generator semantics (both narrowing and matching versions) are soundness and completeness with respect to the predicate semantics \$3.6; informally, whenever we use a Luck program to generate a valuation that satisfies some predicate, the valuation will satisfy the boolean predicate semantics (soundness), and it will generate every possible satisfying valuation with non-zero probability (completeness).

\subsection{Syntax, Typing, and Predicate Semantics}

The syntax of Core Luck is given in Fig. 2 Except for the last line in the definitions of values and expressions, it is a standard simply typed call-by-value lambda calculus with sums, products, and isorecursive types. We include recursive lambdas for convenience in examples, although in principle they could be encoded using recursive types.

Values include unit, pairs of values, sum constructors ( $L$ and $R$ ) applied to values (and annotated with types, to eliminate ambiguity), first class (potentially)recursive functions (rec), foldannotated values indicating where an iso-recursive type should be "folded," and unknowns drawn from an infinite set. The standard expression forms include variables, unit, functions, function applications, pairs with a single-branch pattern-matching construct for deconstructing them, value tagging $(L$ and $R$ ), pattern matching on tagged values, and fold/unfold. The nonstandard additions are unknowns $(u)$, instantiation $\left(e \leftarrow\left(e_{1}, e_{2}\right)\right)$, sample $(! e)$ and after $\left(e_{1} ; e_{2}\right)$ expressions.

The "after" operator, written with a backwards semicolon, evaluates both $e_{1}$ and $e_{2}$ in sequence. However, unlike the standard sequencing operator $e_{1} ; e_{2}$, the result of $e_{1} ; e_{2}$ is the result of $e_{1}$; the expression $e_{2}$ is evaluated just for its side-effects. For example, the sample-after expression e $\mathrm{m}$ of the previous section is desugared to a combination of sample and after: $e: ! x$. If we evaluate this snippet in a context where $x$ is bound to some unknown $u$, then the expression $e$ is evaluated first, refining the domain of $u$ (amongst other unknowns); then the sample expression ! $u$ is evaluated for its side effect, instantiating $u$ to a uniformly generated value from its domain; and finally the result of $e$ is returned as the result of the whole expression. A reasonable way to implement $e_{1} ; e_{2}$ using standard lambda abstractions would be as $\left(\lambda x .\left(\lambda_{-} . x\right) e_{2}\right) e_{1}$. However, there is a slight difference in the semantics of this encoding compared to our intended semantics-we will return to this point in $\$ 3.4$ 


$$
\begin{aligned}
& \text { T-Var } \frac{x: T \in \Gamma}{\Gamma \vdash x: T} \quad \text { T-Unit } \overline{\Gamma \vdash(): 1} \\
& \text { T-Abs } \frac{\Gamma, x: T_{1}, f: T_{1} \rightarrow T_{2} \vdash e_{2}: T_{2}}{\Gamma \vdash \operatorname{rec}\left(f: T_{1} \rightarrow T_{2}\right) x=e_{2}: T_{1} \rightarrow T_{2}} \\
& \text { T-App } \frac{\Gamma \vdash e_{0}: T_{1} \rightarrow T_{2} \quad \Gamma \vdash e_{1}: T_{1}}{\Gamma \vdash\left(e_{0} e_{1}\right): T_{2}} \\
& \text { T-Pair } \frac{\Gamma \vdash e_{1}: T_{1} \quad \Gamma \vdash e_{2}: T_{2}}{\Gamma \vdash\left(e_{1}, e_{2}\right):\left(T_{1} \times T_{2}\right)} \\
& \Gamma \vdash e:\left(T_{1} \times T_{2}\right) \\
& \text { T-CasePair } \frac{\Gamma, x: T_{1}, y: T_{2} \vdash e^{\prime}: T}{\Gamma \vdash \text { case e of }(x, y) \rightarrow e^{\prime}: T} \\
& \text { T-L } \frac{\Gamma \vdash e: T_{1}}{\Gamma \vdash L_{T_{1}+T_{2}} e: T_{1}+T_{2}} \\
& \text { T-R } \frac{\Gamma \vdash e: T_{2}}{\Gamma \vdash R_{T_{1}+T_{2}} e: T_{1}+T_{2}} \\
& \Gamma \vdash e: T_{1}+T_{2} \\
& \text { T-Case } \frac{\Gamma, x: T_{1} \vdash e_{1}: T \quad \Gamma, y: T_{2} \vdash e_{2}: T}{\Gamma \vdash \text { case e of }\left(\text { inl } x \rightarrow e_{1}\right)\left(\text { inr } y \rightarrow e_{2}\right): T} \\
& \text { T-Fold } \frac{U=\mu X . T_{1} \quad \Gamma \vdash e_{1}: T_{1}[U / X]}{\Gamma \vdash \text { fold }_{U} e_{1}: U} \\
& \text { T-Unfold } \frac{U=\mu X . T_{1} \quad \Gamma \vdash e_{1}: U}{\Gamma \vdash \text { unfold }_{U} e_{1}: T_{1}[U / X]}
\end{aligned}
$$

Figure 3. Standard Typing Rules

Weight annotations like the ones in the bst example can be desugared using instantiation expressions. For example, assuming a standard encoding of binary search trees $($ Tree $=\mu X .1+i n t \times$ $X \times X)$ and naturals, plus syntactic sugar for constant naturals:

case $\left(\right.$ unfold $_{\text {Tree }}$ tree $\leftarrow(1$, size $\left.)\right)$ of $(L x \rightarrow \ldots)(R y \rightarrow \ldots)$

Most of the typing rules are standard (these can be found in Fig. 3 ) The four non-standard rules are given in Fig. 4 Unknowns are typed: each will be associated with a domain (set of values) drawn from a type $\bar{T}$ that does not contain arrows. Luck does not support constraint solving over functional domains (which would require something like higher-order unification), and the restriction of unknowns to non-functional types reflects this. To remember the types of unknowns, we extend the typing context to include a component $U$, a map from unknowns to non-functional types. When the variable typing environment $\Gamma=\varnothing$, we write $U \vdash e: T$ as a shorthand for $\varnothing ; U \vdash e: T$. The rules for the standard constructs in Fig. 3 are as expected (adding $U$ everywhere). An unknown $u$ has type $\bar{T}$ if $U(u)=\bar{T}$. If $e_{1}$ and $e_{2}$ are well typed, then $e_{1} ; e_{2}$ shares the type of $e_{1}$. An instantiation expression $e \leftarrow\left(e_{l}, e_{r}\right)$ is well typed if $e$ has sum type $\bar{T}_{1}+\bar{T}_{2}$ and $e_{l}$ and $e_{r}$ are natural numbers. A sample expression !e has the (nonfunctional) type $\bar{T}$ when $e$ has type $\bar{T}$.

The predicate semantics for Core Luck, written $e \Downarrow v$, are defined as a big-step operational semantics. We assume that $e$ is closed with respect to ordinary variables and free of unknowns. The rules for the standard constructs are unsurprising (Fig. 5). The only non-standard rules are the ones for narrow, sample and after

$$
\begin{aligned}
& \text { T-U } \frac{U(u)=\bar{T}}{\Gamma ; U \vdash u: \bar{T}} \quad \text { T-After } \frac{\Gamma ; U \vdash e_{1}: T_{1} \quad \Gamma ; U \vdash e_{2}: T_{2}}{\Gamma ; U \vdash e_{1} ; e_{2}: T_{1}} \\
& \Gamma ; U \vdash e: \bar{T}_{1}+\bar{T}_{2} \\
& \text { T-Bang } \frac{\Gamma ; U \vdash e: \bar{T}}{\Gamma ; U \vdash ! e: \bar{T}} \quad \text { T-Narrow } \frac{\Gamma ; U \vdash e_{l}: \text { nat } \quad \Gamma \vdash e_{r}: \text { nat }}{\Gamma ; U \vdash e \leftarrow\left(e_{l}, e_{r}\right): \bar{T}_{1}+\bar{T}_{2}} \\
& \text { nat }:=\mu X .1+X
\end{aligned}
$$

Figure 4. Typing Rules for Nonstandard Constructs

$$
\begin{aligned}
& \text { P-Val } \frac{i s_{-} v a l u e v}{v \Downarrow v} \\
& e_{0} \Downarrow\left(\operatorname{rec}\left(f: T_{1} \rightarrow T_{2}\right) x=e_{2}\right) \\
& e_{1} \Downarrow v_{1} \\
& \text { P-App } \frac{e\left[\left(\operatorname{rec}\left(f: T_{1} \rightarrow T_{2}\right) x=e_{2}\right) / f, v_{1} / x\right] \Downarrow v}{\left(e_{0} e_{1}\right) \Downarrow v} \\
& \text { P-Pair } \frac{e_{1} \Downarrow v_{1} \quad e_{2} \Downarrow v_{2}}{\left(e_{1}, e_{2}\right) \Downarrow\left(v_{1}, v_{2}\right)} \\
& e \Downarrow\left(v_{1}, v_{2}\right) \\
& \text { P-CasePair } \frac{e^{\prime}\left[v_{1} / x, v_{2} / y\right] \Downarrow v}{\text { case e of }(x, y) \rightarrow e^{\prime} \Downarrow v} \\
& \text { P-L } \frac{e \Downarrow v}{L_{T} e \Downarrow L_{T} v} \\
& \mathbf{P}-\mathbf{R} \frac{e \Downarrow v}{R_{T} e \Downarrow R_{T} v} \\
& \text { P-Case-L } \frac{e \Downarrow L_{T} v \quad e_{1}[v / x] \Downarrow v_{1}}{\text { case e of }\left(L x \rightarrow e_{1}\right)\left(R y \rightarrow e_{2}\right) \Downarrow v_{1}} \\
& \text { P-Case-R } \frac{e \Downarrow R_{T} v}{e_{2}[v / y] \Downarrow v_{2}} \\
& \text { P-Fold } \frac{e \Downarrow v}{\text { fold }_{S} e \Downarrow \text { fold }_{S} v} \\
& \text { P-Unfold } \frac{e \Downarrow \text { fold }_{T} v}{\text { unfold }_{T} e \Downarrow v}
\end{aligned}
$$

Figure 5. Predicate Semantics for Standard Core Luck Constructs

expressions, which are essentially ignored (Fig. 6). With the predicate semantics we can implement a naive generate-and-test method for generating valuations satisfying some predicate by generating arbitrary well-typed valuations and filtering out those for which the predicate does not evaluate to True.

\subsection{Constraint Sets}

The rest of this section develops an alternative probabilistic generator semantics for Core Luck. This semantics will use constraint sets $\kappa \in \mathcal{C}$ to describe the possible values that unknowns can take. 


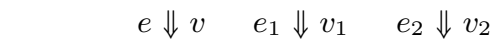

$$
\begin{aligned}
& \text { P-Narrow } \frac{\llbracket v_{1} \rrbracket>0 \quad \llbracket v_{2} \rrbracket>0}{e \leftarrow\left(e_{1}, e_{2}\right) \Downarrow v} \quad \text { P-Bang } \frac{e \Downarrow v}{! e \Downarrow v} \\
& \text { P-After } \frac{e_{1} \Downarrow v_{1} \quad e_{2} \Downarrow v_{2}}{e_{1} ; e_{2} \Downarrow v_{1}} \\
& \llbracket \text { fold }_{\text {nat }}\left(L_{1+\text { nat }}()\right) \rrbracket=0 \\
& \llbracket \text { fold }_{\text {nat }}\left(R_{1+\text { nat }} v\right) \rrbracket=1+\llbracket v \rrbracket
\end{aligned}
$$

Figure 6. Predicate Semantics for Nonstandard Constructs

For the moment, we leave the implementation of constraint sets open (the one used by our prototype interpreter is described in $\$ 4$, simply requiring that they support the following operations:

$$
\begin{array}{lll}
\llbracket \cdot \rrbracket & :: & \mathcal{C} \rightarrow \text { Set Valuation } \\
U & :: & \mathcal{C} \rightarrow \text { Map } \mathcal{U} \bar{T} \\
\text { fresh } & :: & \mathcal{C} \rightarrow \bar{T}^{*} \rightarrow\left(\mathcal{C} \times \mathcal{U}^{*}\right) \\
\text { unify } & :: & \mathcal{C} \rightarrow \text { Val } \rightarrow \text { Val } \rightarrow \mathcal{C} \\
\text { SAT } & :: & \mathcal{C} \rightarrow \text { Bool } \\
{[\cdot]} & :: & \mathcal{C} \rightarrow \mathcal{U} \rightarrow \text { Maybe Val } \\
\text { sample } & :: & \mathcal{C} \rightarrow \mathcal{U} \rightarrow \mathcal{C}^{*}
\end{array}
$$

Here we describe these operations informally, deferring technicalities until after we have presented the generator semantics \$3.6.

A constraint set $\kappa$ denotes a set of valuations $(\llbracket \kappa \rrbracket)$, representing the solutions to the constraints. Constraint sets also carry type information about existing unknowns: $U(\kappa)$ is a mapping from $\kappa$ 's unknowns to types. A constraint set $\kappa$ is well typed $(\vdash \kappa)$ if, for every valuation $\sigma$ in the denotation of $\kappa$ and every unknown $u$ bound in $\sigma$, the type map $U(\kappa)$ contains $u$ and $\varnothing ; U(\kappa) \vdash \sigma(u)$ : $U(\kappa)(u)$.

$$
\forall(\sigma \in \llbracket \kappa \rrbracket)(u \in \sigma) . u \in U(\kappa) \wedge \varnothing ; U(\kappa) \vdash \sigma(u): U(\kappa)(u)
$$

Many of the semantic rules will need to introduce fresh unknowns. The fresh function takes as inputs a constraint set $\kappa$ and a sequence of (non-functional) types of length $k$; it draws the next $k$ unknowns (in some deterministic order) from the infinite set $\mathcal{U}$ and extends $U(\kappa)$ with the respective bindings.

The main way constraints are introduced during evaluation is unification. Given a constraint set $\kappa$ and two values, each potentially containing unknowns, unify updates $\kappa$ to preserve only those valuations in which the values match.

$S A T$ is a total predicate that holds on constraint sets whose denotation contains at least one valuation. The totality requirement implies that our constraints must be decidable.

The value-extraction function $\kappa[u]$ returns an optional (nonunknown) value: if in the denotation of $\kappa$, all valuations map $u$ to the same value $v$, then that value is returned (written $\{v\}$ ); otherwise nothing (written $\varnothing$ ).

The sample operation is used to implement sample expressions (!e): given a constraint set $\kappa$ and an unknown $u \in U(\kappa)$, it returns a list of constraint sets representing all possible concrete choices for $u$, in all of which $u$ is completely determined-that is $\forall \kappa \in($ sample $\kappa u) . \exists v . \kappa[u]=\{v\}$. To allow for reasonable implementations of this interface, we maintain an invariant that the input unknown to sample will always have a finite denotation; thus, the resulting list is also finite.

\subsection{Narrowing Semantics}

As a first step toward a semantics for Core Luck that incorporates both constraint solving and local instantiation, we define a simpler narrowing semantics. This semantics is of some interest in its own right, in that it extends traditional "needed narrowing" with explicit probabilistic instantiation points, but its role here is as a subroutine of the matching semantics in $\$ 3.4$

The narrowing evaluation judgment takes as inputs an expression $e$ and a constraint set $\kappa$. As in the predicate semantics, evaluating $e$ returns a value $v$, but now it also depends on a constraint set $\kappa$ and returns a new constraint set $\kappa^{\prime}$. The latter is intuitively a refinement of $\kappa$-i.e., evaluation will only remove valuations.

$$
e=\kappa \Downarrow_{q}^{t} \kappa^{\prime} \models v
$$

The semantics is annotated with a representation of the sequence of random choices made during evaluation, in the form of a trace $t$. A trace is a sequence of choices: integer pairs $(m, n)$ with $0 \leqslant m<n$, where $n$ denotes the number of possibilities chosen among and $m$ is the index of the one actually taken. We write $\epsilon$ for the empty trace and $t \cdot t^{\prime}$ for the concatenation of two traces. We also annotate the judgment with the probability $q$ of making the choices represented in the trace. Recording traces is useful after the fact in calculating the total probability of some given outcome of evaluation (which may be reached by many different derivations). Traces play no role in determining how evaluation proceeds. We model probability distributions using rational numbers $q \in(0,1] \cap$ $\mathbb{Q}$, for simplicity in the Coq formalization.

We maintain the invariant that the input constraint set $\kappa$ is well typed and that the input expression $e$ is well typed with respect to an empty variable context and the unknown context $U(\kappa)$. Another invariant is that every constraint set $\kappa$ that appears as input to a judgment is satisfiable and the restriction of its denotation to the unknowns in $e$ is finite. These invariants are established at the top-level (see \$4). The finiteness invariant ensures the output of sample will always be a finite collection (and therefore the probabilities involved will be positive rational numbers. Moreover, they guarantee termination of constraint solving, as we will see in $\$ 3.4$ Finally, we assume that the type of every expression has been determined by an initial type-checking phase. We write $e^{T}$ to show that $e$ has type $T$. This information is used in the semantic rules to provide types for fresh unknowns.

The narrowing semantics is given in Fig. 7 for the standard constructs and in Fig. 8 for instantiation expressions; Fig. 10 and Fig. 9 give some auxiliary definitions. Most of the rules are intuitive. A common pattern is sequencing two narrowing judgments $e_{1}=\kappa \Downarrow_{q_{1}}^{t_{1}} \kappa_{1} \models v$ and $e_{2}=\kappa_{1} \Downarrow_{q_{2}}^{t_{2}} \kappa_{2} \models v$. The constraint-set result of the first narrowing judgment $\left(\kappa_{1}\right)$ is given as input to the second, while traces and probabilities are accumulated by concatenation $\left(t_{1} \cdot t_{2}\right)$ and multiplication $\left(q_{1} * q_{2}\right)$. We now explain the rules in detail.

Rule N-Base is the base case of the evaluation relation, handling values that are not handled by other rules by returning them as-is. No choices are made, so the probability of the result is 1 and the trace is empty.

Rule N-Pair: To evaluate $\left(e_{1}, e_{2}\right)$ given a constraint set $\kappa$, we sequence the derivations for $e_{1}$ and $e_{2}$.

Rules N-CasePair-P, N-CasePair-U: To evaluate the pair elimination expression case e of $(x, y) \rightarrow e^{\prime}$ in a constraint set $\kappa$, we first evaluate $e$ in $\kappa$. Typing ensures that the resulting value is either a pair or an unknown. If it is a pair (N-CasePair-P), we substitute its components for $x$ and $y$ in $e^{\prime}$ and continue evaluating. If it is an unknown $u$ of type $\bar{T}_{1} \times \bar{T}_{2}$ (N-CasePair-U), we first use $\bar{T}_{1}$ and $\bar{T}_{2}$ as types for fresh unknowns $u_{1}, u_{2}$ and remember the constraint that the pair $\left(u_{1}, u_{2}\right)$ must unify with $u$. We then proceed as above, this time substituting $u_{1}$ and $u_{2}$ for $x$ and $y$. 


$$
\begin{gathered}
\text { N-Base } \frac{v=() \vee v=\left(r e c\left(f: T_{1} \rightarrow T_{2}\right) x=e^{\prime}\right) \vee v \in \mathcal{U}}{v=\kappa \Downarrow_{1}^{\epsilon} \kappa \models v} \\
\mathbf{N}-\text { Pair } \frac{e_{1}=\kappa \Downarrow_{q_{1}}^{t_{1}} \kappa_{1} \models v_{1} \quad e_{2}=\kappa_{1} \Downarrow_{q_{2}}^{t_{2}} \kappa_{2} \models v_{2}}{\left(e_{1}, e_{2}\right)=\kappa \Downarrow_{q_{1} * q_{2}}^{t_{1} \cdot t_{2}} \kappa_{2} \models\left(v_{1}, v_{2}\right)} \\
e=\kappa \Downarrow_{q}^{t} \kappa_{a} \models\left(v_{1}, v_{2}\right)
\end{gathered}
$$

N-CasePair-P $\quad e^{\prime}\left[v_{1} / x, v_{2} / y\right]=\kappa_{a} \Downarrow_{q^{\prime}}^{t^{\prime}} \kappa^{\prime} \models v$

$$
\begin{gathered}
\text { case e of }(x, y) \rightarrow e^{\prime}=\kappa \Downarrow_{q * q^{\prime}}^{t \cdot t^{\prime}} \kappa^{\prime} \models v \\
e=\kappa \Downarrow_{q}^{t} \kappa_{a} \models u \\
\left(\kappa_{b},\left[u_{1}, u_{2}\right]\right)=\text { fresh } \kappa_{a}\left[\bar{T}_{1}, \bar{T}_{2}\right] \\
\kappa_{c}=\text { unify } \kappa_{b}\left(u_{1}, u_{2}\right) u
\end{gathered}
$$

N-CasePair-U $\quad e^{\prime}\left[u_{1} / x, u_{2} / y\right]=\kappa_{c} \Downarrow_{q^{\prime}}^{t^{\prime}} \kappa^{\prime} \models v$

case $e^{\bar{T}_{1} \times \bar{T}_{2}}$ of $(x, y) \rightarrow e^{\prime}=\kappa \Downarrow_{q * q^{\prime}}^{t \cdot t^{\prime}} \kappa^{\prime} \models v$

N-L $\frac{e=\kappa \Downarrow_{q}^{t} \kappa^{\prime} \models v}{L_{T_{1}+T_{2}} e=\kappa \Downarrow_{q}^{t} \kappa^{\prime} \models L_{T_{1}+T_{2}} v}$

$\mathbf{N}-\mathbf{R} \frac{e=\kappa \Downarrow_{q}^{t} \kappa^{\prime} \models v}{R_{T_{1}+T_{2}} e=\kappa \Downarrow_{q}^{t} \kappa^{\prime} \models R_{T_{1}+T_{2}} v}$

$e=\kappa \Downarrow_{q}^{t} \kappa_{a} \models L_{T} v_{l}$

N-Case-L $\quad e_{l}\left[v_{l} / x_{l}\right]=\kappa_{a} \Downarrow_{q^{\prime}}^{t^{\prime}} \kappa^{\prime} \models v$

case e of $\left(L x_{l} \rightarrow e_{l}\right)\left(R x_{r} \rightarrow e_{r}\right)=\kappa \Downarrow_{q * q^{\prime}}^{t \cdot t^{\prime}} \kappa^{\prime} \models v$

$e=\kappa \Downarrow_{q}^{t} \kappa_{a} \models R_{T} v_{r}$

N-Case-R $\quad e_{r}\left[v_{r} / x_{r}\right]=\kappa_{a} \Downarrow_{q^{\prime}}^{t^{\prime}} \kappa^{\prime} \models v$

case e of $\left(L x_{l} \rightarrow e_{l}\right)\left(R x_{r} \rightarrow e_{r}\right)=\kappa \Downarrow_{q * q^{\prime}}^{t \cdot t^{\prime}} \kappa^{\prime} \models v$

$e=\kappa \Downarrow_{q_{1}}^{t_{1}} \kappa_{a} \models u$

$\left(\kappa_{0},\left[u_{l}, u_{r}\right]\right)=$ fresh $\kappa_{a}\left[\bar{T}_{l}, \bar{T}_{r}\right]$

$\kappa_{l}=$ unify $\kappa_{0} u\left(L_{\bar{T}_{l}+\bar{T}_{r}} u_{l}\right) \quad \kappa_{r}=$ unify $\kappa_{0} u\left(R_{\bar{T}_{l}+\bar{T}_{r}} u_{r}\right)$ choose $1 \kappa_{l} 1 \kappa_{r} \rightarrow{ }_{q_{2}}^{t_{2}} i$

N-Case-U $\quad e_{i}\left[u_{i} / x_{i}\right]=\kappa_{i} \Downarrow_{q_{3}}^{t_{3}} \kappa^{\prime} \models v$

$$
\begin{aligned}
& \text { case } e^{\bar{T}_{l}+\bar{T}_{r}} \text { of }\left(L x_{l} \rightarrow e_{l}\right)\left(R x_{r} \rightarrow e_{r}\right)=\kappa \Downarrow_{q_{1} * q_{2} * q_{3}}^{t_{1} \cdot t_{2} \cdot t_{3}} \kappa^{\prime} \models v \\
& e_{0}=\kappa \Downarrow_{q_{0}}^{t_{0}} \kappa_{a} \models\left(\operatorname{rec}\left(f: T_{1} \rightarrow T_{2}\right) x=e_{2}\right) \\
& \text { N-App } \quad e_{1}=\kappa_{a} \Downarrow_{q_{1}}^{t_{1}} \kappa_{b} \models v_{1} \\
& \frac{e_{2}\left[\left(\operatorname{rec}\left(f: T_{1} \rightarrow T_{2}\right) x=e_{2}\right) / f, v_{1} / x\right]=\kappa_{b} \Downarrow_{q_{2}}^{t_{2}} \kappa^{\prime} \models v}{\left(e_{0} e_{1}\right)=\kappa \Downarrow_{q_{0} * q_{1} * q_{2}}^{t_{0} \cdot t_{1} \cdot t_{2}} \kappa^{\prime} \models v} \\
& \text { N-Fold } \frac{e=\kappa \Downarrow_{q}^{t} \kappa^{\prime} \models v}{\text { fold }_{T} e=\kappa \Downarrow_{q}^{t} \kappa^{\prime} \models \text { fold }_{T} v} \\
& \text { N-Unfold-F } \frac{e=\kappa \Downarrow_{q}^{t} \kappa^{\prime} \models \text { fold }_{T} v}{\text { unfold }_{T} e=\kappa \Downarrow_{q}^{t} \kappa^{\prime} \models v} \\
& e=\kappa \Downarrow_{q}^{t} \kappa_{a} \models u \\
& \left(\kappa_{b}, u^{\prime}\right)=\text { fresh } \kappa_{a} T[\mu X . T / X] \\
& \text { N-Unfold-U } \frac{\kappa^{\prime}=\text { unify } \kappa_{b} u\left(\text { fold }_{\mu X . T} u^{\prime}\right)}{\text { unfold }_{\mu X . T} e=\kappa \Downarrow_{q}^{t} \kappa^{\prime} \models u^{\prime}}
\end{aligned}
$$

Figure 7. Narrowing Semantics of Standard Core Luck Constructs

$$
\begin{aligned}
& \mathbf{N}-\operatorname{After} \frac{e_{1}=\kappa \Downarrow_{q_{1}}^{t_{1}} \kappa_{1} \models v_{1} \quad e_{2}=\kappa_{1} \Downarrow_{q_{2}}^{t_{2}} \kappa_{2} \models v_{2}}{e_{1} ; e_{2}=\kappa \Downarrow_{q_{1} * q_{2}}^{t_{1} \cdot t_{2}} \kappa_{2} \models v_{1}} \\
& \text { N-Bang } \frac{e=\kappa \Downarrow_{q}^{t} \kappa_{a} \models v \quad \text { sample } V \kappa_{a} v \Rightarrow{ }_{q^{\prime}}^{t^{\prime}} \kappa^{\prime}}{! e=\kappa \Downarrow_{q * q^{\prime}}^{t \cdot t^{\prime}} \kappa^{\prime} \models v} \\
& e=\kappa \Downarrow_{q}^{t} \kappa_{a} \models v
\end{aligned}
$$

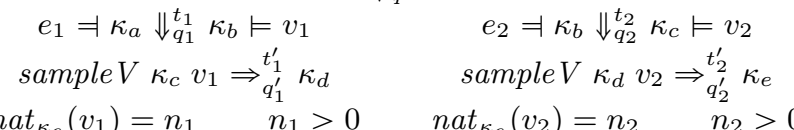

$$
\begin{aligned}
& \left(\kappa_{0},\left[u_{1}, u_{2}\right]\right)=\text { fresh } \kappa_{e}\left[\bar{T}_{1}, \bar{T}_{2}\right] \\
& \kappa_{l}=\text { unify } \kappa_{0} v\left(L_{\bar{T}_{1}+\bar{T}_{2}} u_{1}\right) \quad \kappa_{r}=\text { unify } \kappa_{0} v\left(R_{\bar{T}_{1}+\bar{T}_{2}} u_{2}\right) \\
& \text { N-Narrow choose } n_{1} \kappa_{l} n_{2} \kappa_{r} \rightarrow{ }_{q^{\prime}}^{t^{\prime}} i \\
& e^{\bar{T}_{1}+\bar{T}_{2}} \leftarrow\left(e_{1}^{n a t}, e_{2}^{n a t}\right)=\kappa \Downarrow_{q * q_{1} * q_{2} * q_{1}^{\prime} * q_{2}^{\prime} * q^{\prime}}^{t \cdot t_{1} \cdot t_{2} \cdot t_{1}^{\prime} \cdot t_{i}^{\prime} \cdot t^{\prime}} \kappa_{i} \models v
\end{aligned}
$$

Figure 8. Narrowing Semantics for Non-Standard Expressions

$$
\begin{array}{cccc}
\frac{S A T\left(\kappa_{1}\right)}{\text { choose } n \kappa_{1} m \kappa_{2} \rightarrow{ }_{n /(n+m)}^{[(0,2)]}} \operatorname{SAT}\left(\kappa_{2}\right) & \frac{\neg S A T\left(\kappa_{1}\right)}{\text { choose } n \kappa_{1} m \kappa_{2} \rightarrow_{1}^{\epsilon} r} \\
\frac{S A T\left(\kappa_{1}\right) \quad S A T\left(\kappa_{2}\right)}{\text { choose } n \kappa_{1} m \kappa_{2} \rightarrow{ }_{m /(n+m)}^{[(1,2)]} r} r & \frac{S A T\left(\kappa_{1}\right) \quad \neg S A T\left(\kappa_{2}\right)}{\text { choose } n \kappa_{1} m \kappa_{2} \rightarrow_{1}^{\epsilon} l}
\end{array}
$$

Figure 9. Auxiliary relation choose

$$
\begin{aligned}
& \frac{\text { sample } \kappa u=S \quad S[m]=\kappa^{\prime}}{\text { sample } V \kappa u \Rightarrow_{1 /|S|}^{[(m,|S|)]} \kappa^{\prime}} \\
& \overline{\text { sample } V \kappa() \Rightarrow_{1}^{\epsilon} \kappa} \quad \frac{\text { sample } V \kappa v \Rightarrow_{q}^{t} \kappa^{\prime}}{\text { sample } V \kappa\left(\text { fold }_{T} v\right) \Rightarrow_{q}^{t} \kappa^{\prime}} \\
& \frac{\text { sample } V \kappa v \Rightarrow_{q}^{t} \kappa^{\prime}}{\text { sample } V \kappa\left(L_{T} v\right) \Rightarrow_{q}^{t} \kappa^{\prime}} \quad \frac{\text { sample } V \kappa v \Rightarrow_{q}^{t} \kappa^{\prime}}{\text { sample } V \kappa\left(R_{T} v\right) \Rightarrow_{q}^{t} \kappa^{\prime}} \\
& \underline{\text { sample } V \kappa v_{1} \Rightarrow{ }_{q_{1}}^{t_{1}} \kappa_{1} \quad \text { sample } V \kappa_{1} v_{2} \Rightarrow_{q_{2}}^{t_{2}} \kappa^{\prime}} \\
& \text { sample } V \kappa\left(v_{1}, v_{2}\right) \Rightarrow_{q_{1} * q_{2}}^{t_{1} \cdot t_{2}} \kappa^{\prime}
\end{aligned}
$$

Figure 10. Auxiliary relation sample $V$

(The first pair rule might appear unnecessary since, even in the case where the scrutinee evaluates to a pair, we could generate unknowns, unify, and substitute, as in N-CasePair-U. However, unknowns in Luck only range over non-functional types $\bar{T}$, so this trick does not work when the type of the $e$ contains arrows.)

The $\mathbf{N}$-CasePair- $\mathbf{U}$ rule also shows how the finiteness invariant is preserved: when we generate the unknowns $u_{1}$ and $u_{2}$, their domains are unconstrained, but before we substitute them into an expression used as "input" to a subderivation, we unify them with the result of a narrowing derivation, which already has a finite representation in $\kappa_{a}$.

Rules N-L, N-R: To evaluate $L_{T_{1}+T_{2}} e$, we evaluate $e$ and tag the resulting value with $L_{T_{1}+T_{2}}$, with the resulting constraint set, trace, and probability unchanged. $R_{T_{1}+T_{2}} e$ is handled similarly . 
Rules N-Case-L,N-Case-R,N-Case-U: As in the pair elimination rule, we first evaluate the discriminee $e$ to a value, which must have one of the shapes $L_{T} v_{l}, R_{T} v_{r}$, or $u \in \mathcal{U}$, thanks to typing. The cases for $L_{T} v_{l}$ (rule N-Case-L) and $R_{T} v_{r}$ (rule N-Case-R) are similar to N-CasePair-P: $v_{l}$ or $v_{r}$ can be directly substituted for $x_{l}$ or $x_{r}$ in $e_{l}$ or $e_{r}$. The unknown case (N-Case-U) is similar to $\mathbf{N}-$ CasePair-U but a bit more complex. Once again $e$ shares with the unknown $u$ a type $\bar{T}_{l}+\bar{T}_{r}$ that does not contain any arrows, so we can generate fresh unknowns $u_{l}, u_{r}$ with types $\bar{T}_{l}, \bar{T}_{r}$. We unify $L_{\bar{T}_{l}+\bar{T}_{r}} v_{l}$ with $u$ to get the constraint set $\kappa_{l}$ and $R_{\bar{T}_{l}+\bar{T}_{r}} v_{r}$ with $u$ to get $\kappa_{r}$. We then use the auxiliary relation choose (Fig. 9], which takes two integers $n$ and $m$ (here equal to 1 ) as well as two constraint sets (here $\kappa_{l}$ and $\kappa_{r}$ ), to select either $l$ or $r$. If exactly one of $\kappa_{l}$ and $\kappa_{r}$ is satisfiable, then choose will return the corresponding index with probability 1 and an empty trace (because no random choice were made). If both are satisfiable, then the resulting index is randomly chosen. Both outcomes are equiprobable (because of the 1 arguments to choose), so the probability is one half in each case. This uniform binary choice is recorded in the trace $t_{2}$ as either $(0,2)$ or $(1,2)$. Finally, we evaluate the expression corresponding to the chosen index, with the corresponding unknown substituted for the variable. The satisfiability checks enforce the invariant that constraint sets are satisfiable, which in turn ensures that $\kappa_{l}$ and $\kappa_{r}$ cannot both be unsatisfiable at the same time, since there must exist at least one valuation in $\kappa_{0}$ that maps $u$ to a value (either $L$ or $R$ ) which ensures that the corresponding unification will succeed.

Rule N-App: To evaluate an application $\left(e_{0} e_{1}\right)$, we first evaluate $e_{0}$ to $r e c\left(f: T_{1} \rightarrow T_{2}\right) x=e_{2}$ (since unknowns only range over arrow-free types $\bar{T}$, the result cannot be an unknown) and its argument $e_{1}$ to a value $v_{1}$. We then evaluate the appropriately substituted body, $e_{2}\left[\left(r e c\left(f: T_{1} \rightarrow T_{2}\right) x=e_{2}\right) / f, v_{1} / x\right]$, and combine the various probabilities and traces appropriately.

Rule N-After is similar to N-Pair; however, the value result of the derivation is that of the first narrowing evaluation, implementing the reverse form of sequencing described in the introduction of this section.

Rule N-Fold is similar to N-L. N-Unfold-F and N-Unfold$\mathbf{U}$ are similar to (though simpler than) $\mathbf{N}$-CasePair-P and $\mathbf{N}$ CasePair-U.

Rule N-Bang: To evaluate ! $e$ we evaluate $e$ to a value $v$, then use the auxiliary relation sample $V$ (Fig. 10) to completely instantiate $v$, walking down the structure of $v$. When unknowns are encountered, sample is used to produce a list of constraint sets $S$; with probability $\frac{1}{|S|}$ (where $|S|$ is the size of the list) we can select the $m$ th constraint set in $S$, for each $0 \leqslant m<|S|$.

Rule N-Narrow is similar to N-Case-U. The main difference is the "weight" arguments $e_{1}$ and $e_{2}$. These are evaluated to values $v_{1}$ and $v_{2}$, and sample $V$ is called to ensure that they are fully instantiated in all subsequent constraint sets, in particular in $\kappa_{e}$. The relation nat $_{\kappa_{e}}\left(v_{1}\right)=n_{1}$ walks down the structure of the value $v_{1}$ (like sample $V$ ) and calculates the unique natural number $n_{1}$ corresponding to $v_{1}$. Specifically, when the input value is an unknown, nat $_{\kappa}(u)=n$ holds if $\kappa[u]=v^{\prime}$ and $\llbracket v \rrbracket=n$, where the notation $\llbracket v \rrbracket$ is defined in Fig. 6 The rest of the rule is the same as $\mathbf{N}$-Case-U, except that the computed weights $n_{1}$ and $n_{2}$ are given as arguments to choose in order to shape the distribution accordingly.

Using the narrowing semantics, we can implement a more efficient method for generating valuations than the naive generateand-test described in Section \$3.1 instead of generating arbitrary valuations we only lazily instantiate a subset of unknowns as we encounter them. This method has the additional advantage that, if a generated valuation yields an unwanted result, the implementation can backtrack to the point of the latest choice, which can drastically improve performance [18].
Unfortunately, using the narrowing semantics in this way can lead to a lot of backtracking. To see why, consider three unknowns, $u_{1}, u_{2}$, and $u_{3}$, and a constraint set $\kappa$ where each unknown has type Bool (i.e., $1+1$ ) and the domain associated with each contains both True and False $\left(L_{1+1}()\right.$ and $\left.R_{1+1}()\right)$. Suppose we want to generate valuations for these three unknowns such that the conjunction $u_{1} \& \& u_{2} \& \& u_{3}$ holds, where $e_{1} \& \& e_{2}$ is shorthand for case $e_{1}$ of $\left(L x \rightarrow e_{2}\right)(R y \rightarrow$ False $)$. If we attempt to evaluate the expression $u_{1} \& \& u_{2} \& \& u_{3}$ using the narrowing semantics, we first apply the $\mathbf{N}$-Case-U rule with $e=u_{1}$. That means that $u_{1}$ will be unified with either $L$ or $R$ (applied to a fresh unknown) with equal probability, leading to a False result for the entire expression $50 \%$ of the time. If we choose to unify $u_{1}$ with an $L$, then we apply the $\mathbf{N}$-Case- $\mathbf{U}$ rule again, returning either False or $u_{3}$ (since unknowns are values-rule N-Base) with equal probability. Therefore, we will have generated a desired valuation only $25 \%$ of the time; we will need to backtrack $75 \%$ of the time.

The problem here is that the narrowing semantics is agnostic to the desired result of the whole computation - we only find out at the very end that we need to backtrack. But we can do better...

\subsection{Matching Semantics}

In this section we present a matching semantics that takes as an additional input a pattern (a value not containing lambdas but possibly containing unknowns)

$$
p::=()|(p, p)| L_{\bar{T}} p\left|R_{\bar{T}} p\right| \text { fold }_{\bar{T}} p \mid u
$$

and propagates this pattern backwards to guide the generation process. By allowing our semantics to look ahead in this way, we can often avoid case branches that lead to non-matching results.

The matching judgment is again a variant of big-step evaluation; it has the form

$$
p \Leftarrow e=\kappa \Uparrow_{q}^{t} \kappa^{?}
$$

where the pattern $p$ can mention the unknowns in $U(\kappa)$ and where the metavariable $\kappa^{?}$ stands for an optional constraint set ( $\varnothing$ or $\{\kappa\}$ ) returned by matching. Returning an option allows us to calculate the probability of backtracking by summing the $q$ 's of all failing derivations. (The combined probability of failures and successes may be less than 1 , because some reduction paths may diverge.)

We keep the invariants from $\$ 3.3$ the input constraint set $\kappa$ is well typed and so is the input expression $e$ (with respect to an empty variable context and $U(\kappa)$ ); moreover $\kappa$ is satisfiable, and the restriction of its denotation to the unknowns in $e$ is finite. To these invariants we add that the input pattern $p$ is well typed in $U(\kappa)$ and that the common type of $e$ and $p$ does not contain any arrows ( $e$ can still contain functions and applications internally; these are handled by calling the narrowing semantics).

The following properties are essential to maintaining these invariants. Whenever the output option has the form $\left\{\kappa^{\prime}\right\}$, then $\kappa^{\prime}$ is satisfiable. This is easily ensured by checking the satisfiability of candidate constraint sets and outputting $\varnothing$ if they are not satisfiable. Moreover, when the output has the form $\left\{\kappa^{\prime}\right\}$, then all the unknowns of $p$ have finite denotations in $\kappa^{\prime}$ (despite them not necessarily having finite denotations in the input constraint set $\kappa$ ).

The evaluation relation appears in Fig. 11 (standard constructs) and Fig. 14 (novel Luck constructs). Additional rules concerning failure propagating cases appear in Fig. 13. while match rules that deal with discriminees containing arrow types appear in Fig. 12 Most of them are largely similar to the narrowing rules, only introducing unifications with target patterns in key places. Several of them rely on the narrowing semantics defined previously.

Rule M-Base: To generate valuations for a unit value or an unknown, we unify $v$ and the target pattern $p$ under the input constraint set $\kappa$. Unlike $\mathbf{N}$-Base, there is no case for functions, since the expression being evaluated must have a non-function type. 
M-Base $\frac{v=() \vee v \in \mathcal{U} \quad \kappa^{\prime}=\text { unify } \kappa v p}{p \Leftarrow v=\kappa \Uparrow_{1}^{\epsilon} \text { if } S A T\left(\kappa^{\prime}\right) \text { then }\left\{\kappa^{\prime}\right\} \text { else } \varnothing}$

$$
\begin{gathered}
\left(\kappa^{\prime},\left[u_{1}, u_{2}\right]\right)=\text { fresh } \kappa\left[\bar{T}_{1}, \bar{T}_{2}\right] \\
\kappa_{0}=\text { unify } \kappa^{\prime}\left(u_{1}, u_{2}\right) p
\end{gathered}
$$

M-Pair $\frac{u_{1} \Leftarrow e_{1}=\kappa_{0} \Uparrow_{q_{1}}^{t_{1}}\left\{\kappa_{1}\right\} \quad u_{2} \Leftarrow e_{2}=\kappa_{1} \Uparrow_{q_{2}}^{t_{2}} \kappa_{2}^{?}}{p \Leftarrow\left(e_{1}^{\bar{T}_{1}}, e_{2}^{\bar{T}_{2}}\right)=\kappa \Uparrow_{q_{1} * q_{2}}^{t_{1} \cdot t_{2}} \kappa_{2}^{?}}$

$$
\left(\kappa_{a},\left[u_{1}, u_{2}\right]\right)=\text { fresh } \kappa\left[\bar{T}_{1}, \bar{T}_{2}\right]
$$

M-CasePair $\quad\left(u_{1}, u_{2}\right) \Leftarrow e=\kappa_{a} \Uparrow_{q_{1}}^{t_{1}}\left\{\kappa_{b}\right\}$

$$
\frac{p \Leftarrow e^{\prime}\left[u_{1} / x, u_{2} / y\right]=\kappa_{b} \Uparrow_{q_{2}}^{t_{2}} \kappa^{?}}{p \Leftarrow \text { case } e^{\bar{T}_{1} \times \bar{T}_{2}} \text { of }(x, y) \rightarrow e^{\prime}=\kappa \Uparrow_{q_{1} * q_{2}}^{t_{1} \cdot t_{2}} \kappa^{?}}
$$

$$
\left(\kappa_{1}, u\right)=\text { fresh } \kappa \bar{T}_{1}
$$$$
\kappa_{2}=\text { unify } \kappa_{1}\left(L_{\bar{T}_{1}+\bar{T}_{2}} u\right) p
$$

M-L-Sat $\frac{S A T\left(\kappa_{2}\right) \quad u \Leftarrow e=\kappa_{2} \Uparrow_{q}^{t} \kappa^{?}}{p \Leftarrow L_{\bar{T}_{1}+\bar{T}_{2}} e=\kappa \Uparrow_{q}^{t} \kappa^{?}}$

$\left(\kappa_{1}, u\right)=$ fresh $\kappa \bar{T}_{2}$

$\kappa_{2}=$ unify $\kappa_{1}\left(R_{\bar{T}_{1}+\bar{T}_{2}} u\right) p$

M-R-Sat $\frac{S A T\left(\kappa_{2}\right) \quad u \Leftarrow e=\kappa_{2} \Uparrow_{q}^{t} \kappa^{?}}{p \Leftarrow R_{\bar{T}_{1}+\bar{T}_{2}} e=1 \kappa \Uparrow_{q}^{t} \kappa^{?}}$

$$
e_{0}=\kappa \Downarrow_{q_{0}}^{t_{0}} \kappa_{0} \models\left(\operatorname{rec} f x=e_{2}\right)
$$$$
e_{1}=\kappa_{0} \Downarrow_{q_{1}}^{t_{1}} \kappa^{\prime} \models v_{1}
$$

$$
\begin{aligned}
& \text { M-App } \frac{p \Leftarrow e_{2}\left[\left(\operatorname{rec} f x=e_{2}\right) / f, v_{1} / x\right]=\kappa^{\prime} \Uparrow_{q_{2}}^{t_{2}} \kappa^{?}}{p \Leftarrow\left(e_{0} e_{1}\right)=\kappa \Uparrow_{q_{0} * q_{1} * q_{2}}^{t_{0} \cdot t_{1} \cdot t_{2}} \kappa^{?}} \\
& \left(\kappa_{1}, u\right)=\text { fresh } \kappa \bar{T}[\mu X . \bar{T} / X] \\
& \kappa_{2}=\text { unify } \kappa_{1}\left(\text { fold }_{\mu X . \bar{T}} u\right) p \\
& \text { M-Fold } \frac{u \Leftarrow e=\kappa_{2} \Uparrow_{q}^{t} \kappa^{?}}{p \Leftarrow \text { fold }_{\mu X . \bar{T}} e=\kappa \Uparrow_{q}^{t} \kappa^{?}} \\
& \operatorname{M-Unfold} \frac{\left(\text { fold }_{\mu X . \bar{T}} p\right) \Leftarrow e=\kappa \Uparrow_{q}^{t} \kappa^{?}}{p \Leftarrow \text { unfold }_{\mu X . \bar{T}} e=\kappa \Uparrow_{q}^{t} \kappa^{?}}
\end{aligned}
$$

Figure 11. Matching Semantics of Standard Core Luck Constructs

Rules M-Pair, M-Pair-Fail: To evaluate $\left(e_{1}, e_{2}\right)$, where $e_{1}$ and $e_{2}$ have types $\bar{T}_{1}$ and $\bar{T}_{2}$, we first generate fresh unknowns $u_{1}$ and $u_{2}$ with these types. We unify the pair $\left(u_{1}, u_{2}\right)$ with the target pattern $p$, obtaining a new constraint set $\kappa^{\prime}$. We then proceed as in N-Pair, evaluating $e_{1}$ against pattern $u_{1}$ and $e_{2}$ against $u_{2}$, threading constraint sets and accumulating traces and probabilities. M-Pair handles the case where the evaluation of $e_{1}$ succeeds, yielding a constraint set $\left\{\kappa_{1}\right\}$, while M-Pair-Fail handles failure: if evaluating $e_{1}$ yields $\varnothing$, the whole computation immediately yields $\varnothing$ as well; $e_{2}$ is not evaluated, and the final trace and probability are $t_{1}$ and $q_{1}$.

Rules M-CasePair, M-CasePair-Fail, M-CasePair-Fun: If the type of the discriminee $e$ contains function types (M-CasePairFun), we narrow $e$ to a pair and substitute its components as in $\mathbf{N}$ CasePair-P, but then we evaluate the resulting expression against the original target pattern $p$. Otherwise $e$ has a type of form $\bar{T}_{1} \times \bar{T}_{2}$ and we proceed as in $\mathbf{N}$-CasePair- $\mathbf{U}$ with a few differences. The unknowns $u_{1}$ and $u_{2}$ are introduced before the evaluation of $e$
$T_{1} \notin \bar{T} \vee T_{2} \notin \bar{T}$

$$
\begin{aligned}
& \text { M-CasePair-Fun } \quad e=\kappa \Downarrow_{q_{1}}^{t_{1}} \kappa_{1} \models\left(v_{1}, v_{2}\right) \\
& \frac{p \Leftarrow e^{\prime}\left[v_{1} / x, v_{2} / y\right]=\kappa_{1} \Uparrow_{q_{2}}^{t_{2}} \kappa_{2}^{?}}{p \Leftarrow \text { case } e^{T_{1} \times T_{2}} \text { of }(x, y) \rightarrow e^{\prime}=\kappa \Uparrow_{q_{1} * q_{2}}^{t_{1} \cdot t_{2}} \kappa_{2}^{?}} \\
& T_{1} \notin \bar{T} \vee T_{2} \notin \bar{T} \\
& e=\kappa \Downarrow_{q_{1}}^{t_{1}} \kappa_{1} \models L_{T_{1}+T_{2}} v_{1} \\
& \text { M-Case-L-Fun } \quad p \Leftarrow e_{1}\left[v_{1} / x_{l}\right]=\kappa_{1} \Uparrow_{q_{1}^{\prime}}^{t_{1}^{\prime}} \kappa^{\text {? }} \text { ? } \\
& p \Leftarrow \text { case } e^{T_{1}+T_{2}} \text { of }\left(L x_{l} \rightarrow e_{1}\right)\left(R x_{r} \rightarrow e_{2}\right)=\kappa \Uparrow_{q_{1} * q_{1}^{\prime}}^{t_{1} \cdot t_{1}^{\prime}} \kappa^{?} \\
& T_{1} \notin \bar{T} \vee T_{2} \notin \bar{T} \\
& e=\kappa \Downarrow_{q_{1}}^{t_{1}} \kappa_{1} \models R_{T_{1}+T_{2}} v_{2} \\
& \text { M-Case-R-Fun } \quad p \Leftarrow e_{2}\left[v_{2} / x_{r}\right]=\kappa_{1} \Uparrow_{q_{1}^{\prime}}^{t_{1}^{\prime}} \kappa^{?} \text { ? } \\
& p \Leftarrow \text { case } e^{T_{1}+T_{2}} \text { of }\left(L x_{l} \rightarrow e_{1}\right)\left(R x_{r} \rightarrow e_{2}\right)=\kappa \Uparrow_{q_{1} * q_{1}^{\prime}}^{t_{1} \cdot t_{1}^{\prime}} \kappa^{?}
\end{aligned}
$$

Figure 12. Matching Semantics for Function Cases

$$
\begin{aligned}
& \left(\kappa^{\prime},\left[u_{1}, u_{2}\right]\right)=\text { fresh } \kappa\left[\bar{T}_{1}, \bar{T}_{2}\right] \\
& \kappa_{0}=\text { unify } \kappa^{\prime}\left(u_{1}, u_{2}\right) p \\
& \text { M-Pair-Fail } \frac{u_{1} \Leftarrow e_{1}=\kappa_{0} \Uparrow_{q_{1}}^{t_{1}} \varnothing}{p \Leftarrow\left(e_{1}^{\bar{T}_{1}}, e_{2}^{\bar{T}_{2}}\right)=\kappa \Uparrow_{q_{1}}^{t_{1}} \varnothing} \\
& \left(\left[u_{1}, u_{2}\right], \kappa_{0}\right)=\text { fresh } \kappa\left[\bar{T}_{1}, \bar{T}_{2}\right] \\
& \text { M-CasePair-Fail } \frac{\left(u_{1}, u_{2}\right) \Leftarrow e=\kappa_{0} \Uparrow_{q_{1}}^{t_{1}} \varnothing}{p \Leftarrow \text { case } e^{\bar{T}_{1} \times \bar{T}_{2}} \text { of }(x, y) \rightarrow e^{\prime}=\kappa \Uparrow_{q_{1}}^{t_{1}} \varnothing} \\
& \text { M-Til-Fail } \frac{p \Leftarrow e_{1}=\kappa \Uparrow_{q_{1}}^{t_{1}} \varnothing}{p \Leftarrow e_{1} ; e_{2}=\kappa \Uparrow_{q_{1}}^{t_{1}} \varnothing} \\
& \left(\kappa_{1}, u\right)=\text { fresh } \kappa \bar{T}_{1} \\
& \kappa_{2}=\text { unify } \kappa_{1}\left(L_{\bar{T}_{1}+\bar{T}_{2}} u\right) p \\
& \text { M-L-UnSat } \frac{\neg S A T\left(\kappa_{2}\right)}{p \Leftarrow L_{\bar{T}_{1}+\bar{T}_{2}} e=\kappa \Uparrow_{1}^{\epsilon} \varnothing} \\
& \left(\kappa_{1}, u\right)=\text { fresh } \kappa \bar{T}_{2} \\
& \kappa_{2}=\text { unify } \kappa_{1}\left(R_{\bar{T}_{1}+\bar{T}_{2}} u\right) p \\
& \text { M-R-UnSat } \frac{\neg S A T\left(\kappa_{2}\right)}{p \Leftarrow R_{\bar{T}_{1}+\bar{T}_{2}} e=\kappa \Uparrow_{1}^{\epsilon} \varnothing}
\end{aligned}
$$

Figure 13. Failure Propagation for Matching Semantics 


$$
\begin{aligned}
& \text { M-After } \frac{p \Leftarrow e_{1}=\kappa \Uparrow_{q_{1}}^{t_{1}}\left\{\kappa_{1}\right\} \quad e_{2}=\kappa_{1} \Downarrow_{q_{2}}^{t_{2}} \kappa_{2} \models v}{p \Leftarrow e_{1} ; e_{2}=\kappa \Uparrow_{q_{1} * q_{2}}^{t_{1} \cdot t_{2}}\left\{\kappa_{2}\right\}} \\
& p \Leftarrow e=\kappa \Uparrow_{q_{1}}^{t_{1}}\left\{\kappa_{1}\right\} \\
& \text { M-Bang } \frac{\text { sample } V \kappa_{1} p \Rightarrow{ }_{q_{2}}^{t_{2}} \kappa^{\prime}}{p \Leftarrow ! e=\kappa \Uparrow_{q_{1} * q_{2}}^{t_{1} \cdot t_{2}}\left\{\kappa^{\prime}\right\}} \\
& \text { M-Bang-Fail } \frac{p \Leftarrow e=\kappa \Uparrow_{q_{1}}^{t_{1}} \varnothing}{p \Leftarrow ! e=\kappa \Uparrow_{q_{1}}^{t_{1}} \varnothing} \\
& p \Leftarrow e=\kappa \Uparrow_{q}^{t}\left\{\kappa_{a}\right\} \\
& e_{1}=\kappa_{a} \Downarrow_{q_{1}}^{t_{1}} \kappa_{b} \models v_{1} \quad e_{2}=\kappa_{b} \Downarrow_{q_{2}}^{t_{2}} \kappa_{c} \models v_{2}
\end{aligned}
$$

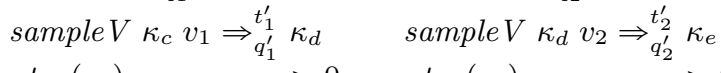

$$
\begin{aligned}
& \operatorname{nat}_{\kappa_{e}}\left(v_{1}\right)=n_{1} \quad n_{1}>0 \quad \operatorname{nat}_{\kappa_{e}}\left(v_{2}\right)=n_{2} \quad n_{2}>0 \\
& \left(\kappa_{0},\left[u_{1}, u_{2}\right]\right)=\text { fresh } \kappa_{e}\left[\bar{T}_{1}, \bar{T}_{2}\right] \\
& \kappa_{l}=\text { unify } \kappa_{0} p\left(L_{\bar{T}_{1}+\bar{T}_{2}} u_{1}\right) \\
& \kappa_{r}=\text { unify } \kappa_{0} p\left(R_{\bar{T}_{1}+\bar{T}_{2}} u_{2}\right) \\
& \text { M-Narrow choose } n_{1} \kappa_{l} n_{2} \kappa_{r} \rightarrow{ }_{q^{\prime}}^{t^{\prime}} i \\
& p \Leftarrow e^{\bar{T}_{1}+\bar{T}_{2}} \leftarrow\left(e_{1}^{n a t}, e_{2}^{n a t}\right)=\kappa \Uparrow_{q * q_{1} * q_{2} * q_{1}^{\prime} * q_{2}^{\prime} * q^{\prime}}^{t \cdot t_{1} \cdot t_{2} \cdot t_{1}^{\prime} \cdot t_{i}^{\prime} \cdot t^{\prime}}\left\{\kappa_{i}\right.
\end{aligned}
$$

$$
\begin{aligned}
& \text { M-Narrow-Fail } \quad p \Leftarrow e=\kappa \Uparrow_{q}^{t} \varnothing \\
& p \Leftarrow e^{\bar{T}_{1}+\bar{T}_{2}} \leftarrow\left(e_{1}^{n a t}, e_{2}^{n a t}\right)=\kappa \Uparrow_{q}^{t} \varnothing
\end{aligned}
$$

Figure 14. Matching Semantics of Nonstandard Core Luck Constructs

to provide a target pattern $\left(u_{1}, u_{2}\right)$. If the evaluation succeeds in yielding $\left\{\kappa_{b}\right\}$ (M-CasePair) we proceed to substitute $u_{1}$ and $u_{2}$ (that now have a finite domain as all pattern unknowns at the resulting constraint sets). If instead evaluation of $e$ yields $\varnothing$ (MCasePair-Fail), the whole computation returns $\varnothing$ immediately.

Rules M-L-Sat, M-R-Sat, M-L-UnSat, M-R-UnSat: To evaluate $L_{\bar{T}_{1}+\bar{T}_{2}} e$, we generate an unknown $u$ of type $\bar{T}_{1}$ and unify $L_{\bar{T}_{1}+\bar{T}_{2}} u$ with the target pattern $p$. If the constraint set obtained is satisfiable (M-L-Sat), we simply evaluate $e$ against the pattern $u$. Otherwise (M-L-UnSat) we immediately return $\varnothing$. The same goes for $R$.

Rules M-App, M-After: To evaluate an application $e_{0} e_{1}$, we use the narrowing semantics to reduce $e_{0}$ to rec $f x=e_{2}$ and $e_{1}$ to a value $v_{1}$, then evaluate $e_{2}\left[\left(\operatorname{rec} f x=e_{2}\right) / f, v_{2} / x\right]$ against the original target pattern $p$ in the matching semantics. In this rule we cannot use a pattern during the evaluation of $e_{1}$ : we do not have any candidates! This is the main reason for introducing the sequencing operator as a primitive $e_{1} ; e_{2}$ instead of encoding it using lambda abstractions. In M-After, we evaluate $e_{1}$ against the target pattern $p$ and then evaluate $e_{2}$ using narrowing, just for its side effects. If we used lambdas to encode sequencing, $e_{1}$ would be narrowed instead, which is not what we want.

Rules M-Fold, M-Unfold: M-Fold is similar to M-Pair, only simpler. To evaluate unfold ${ }_{\mu X} \bar{T} \quad e$ with pattern $p$, M-Unfold simply evaluates $e$ with the pattern fold ${ }_{\mu X} . \bar{T} p$.

Rules M-Bang, M-Bang-Fail: This rule is very similar to $\mathbf{N}$ Bang. We first evaluate $e$ against pattern $p$. If that succeeds we proceed to use the same auxiliary relation sample $V$ as in $\mathbf{N}$-Bang (defined in Fig. 10. Otherwise, the whole computation returns $\varnothing$.

Rules M-Narrow, M-Narrow-Fail: Like in M-Bang, we propagate the pattern $p$ and evaluate $e$ against it. After checking that the resulting constraint set option is not $\varnothing$, we proceed exactly as in N-Narrow.

Rules M-Case-L-Fun, M-Case-R-Fun: If the type of the discriminee $e$ contains function types (meaning it cannot be written as $\bar{T}_{1}+\bar{T}_{2}$ ), we proceed as in N-Case-L and $\mathbf{N}$-Case-R, except in the final evaluation we match the expression against $p$.

The interesting rules are the ones for case when the type of the scrutinee does not contain functions. For these rules, we can actually use the patterns to guide the generation that occurs during the evaluation of the scrutinee as well. We model the behavior of constraint solving: instead of choosing which branch to follow with some probability (50\% in N-Case-U), we evaluate both branches, just like a constraint solver would exhaustively search the entire domain.

Before looking at the rules in detail, we need to extend the constraint set interface with two new functions:

$$
\begin{array}{lll}
\text { rename } & :: & \mathcal{U}^{*} \rightarrow \mathcal{C} \rightarrow \mathcal{C} \\
\text { union } & :: & \mathcal{C} \rightarrow \mathcal{C} \rightarrow \mathcal{C}
\end{array}
$$

The rename operation freshens a constraint set by replacing all the unknowns in a given sequence with freshly generated ones (of the same type). The union of two constraint sets intuitively denotes the union of their corresponding denotations.

The four case rules with function-free types appear in Fig. 15 We independently evaluate $e$ against both an $L$ pattern and an $R$ pattern. If both of them yield failure, then the whole evaluation yields failure (M-Case-4). If exactly one succeeds, we evaluate just the corresponding branch (M-Case-2 or M-Case-3). If both succeed (M-Case-1), we evaluate both branch bodies and combine the results with union. We use rename to avoid conflicts, since we may generate the same fresh unknowns while independently computing $\kappa_{a}^{?}$ and $\kappa_{b}^{?}$.

If desired, the user can ensure that only one branch will be executed by using an instantiation expression before the case is reached. Since $e$ will then begin with a concrete constructor, only one of the evaluations of $e$ against the patterns $L$ and $R$ will succeed, and only the corresponding branch will be executed.

The M-Case-1 rule is the second place where the need for finiteness of the restriction of $\kappa$ to the input expression $e$ arises. In order for the semantics to terminate in the presence of (terminating) recursive calls, it is necessary that the domain be finite. To see this, consider a simple recursive predicate that holds for every number:

$$
\begin{aligned}
& \text { rec }(f: \text { nat } \rightarrow \text { bool }) u= \\
& \quad \text { case unfold } \\
& \text { nat } \\
& u \text { of }(L x \rightarrow \text { True })(R y \rightarrow(f y))
\end{aligned}
$$

Even though $f$ terminates in the predicate semantics for every input $u$, if we allow a constraint set to map $u$ to the infinite domain of all natural numbers, the matching semantics will not terminate. While this finiteness restriction feels a bit unnatural, we have not found it to be a problem in practice- - see $\$ 4$

\subsection{Example}

To show how all this works, let's trace the main steps of the matching derivations of two given expressions against the pattern True in a given constraint set. We will also extract probability distributions about optional constraint sets from these derivations.

We are going to evaluate $A:=(0<u \& \& u<4)$ : ! u and $B:=(0<u ; ! u) \& \& u<4$ against the pattern True in a constraint set $\kappa$, in which $u$ is independent from other unknowns and its possible values are $0, \ldots, 9$. Similar expressions were introduced as examples in $\$ 2$, the results we obtain here confirm the intuitive explanation given there.

Recall that the conjunction expression $e_{1} \& \& e_{2}$ is shorthand for case $e_{1}$ of $\left(L a \rightarrow e_{2}\right)(R b \rightarrow$ False $)$, and that we are using a standard Peano encoding of naturals: $n a t=\mu X .1+X$. We elide folds for brevity. The inequality $a<b$ can be encoded as $l t a b$, 


$$
\begin{aligned}
& \left(\kappa_{0},\left[u_{1}, u_{2}\right]\right)=\text { fresh } \kappa\left[\bar{T}_{1}, \bar{T}_{2}\right] \\
& \left(L_{\bar{T}_{1}+\bar{T}_{2}} u_{1}\right) \Leftarrow e=\kappa_{0} \Uparrow_{q_{1}}^{t_{1}}\left\{\kappa_{1}\right\} \\
& \left(R_{\bar{T}_{1}+\bar{T}_{2}} u_{2}\right) \Leftarrow e=\kappa_{0} \Uparrow_{q_{2}}^{t_{2}}\left\{\kappa_{2}\right\} \\
& p \Leftarrow e_{1}\left[u_{1} / x_{l}\right]=\kappa_{1} \Uparrow_{q_{1}^{\prime}}^{t_{1}^{\prime}} \kappa_{a}^{?} \quad p \Leftarrow e_{2}\left[u_{2} / y_{r}\right]=\kappa_{2} \Uparrow_{q_{2}^{\prime}}^{t_{2}^{\prime}} \kappa_{b}^{?} \\
& \text { M-Case-1 } \quad \kappa^{?}=\text { combine } \kappa_{0} \kappa_{a}^{?} \kappa_{b}^{?} \\
& p \Leftarrow \text { case } e^{\bar{T}_{1}+\bar{T}_{2}} \text { of }\left(L x_{l} \rightarrow e_{1}\right)\left(R y_{r} \rightarrow e_{2}\right)=\kappa \\
& \Uparrow_{q_{1} * q_{2} * q_{1}^{\prime} * q_{2}^{\prime}}^{t_{1} \cdot t_{2} \cdot t_{1}^{\prime} \cdot t^{\prime}} \text { ? } \\
& \text { combine } \kappa\left\{\kappa_{1}\right\} \varnothing=\left\{\kappa_{1}\right\} \\
& \text { combine } \kappa \varnothing\left\{\kappa_{2}\right\}=\left\{\kappa_{2}\right\} \\
& \text { combine } \kappa\left\{\kappa_{1}\right\}\left\{\kappa_{2}\right\}= \\
& \left\{\text { union } \kappa_{1}\left(\text { rename }\left(U\left(\kappa_{1}\right)-U(\kappa)\right) \kappa_{2}\right)\right\} \\
& \begin{array}{c}
\left(\kappa_{0},\left[u_{1}, u_{2}\right]\right)=\text { fresh } \kappa\left[\bar{T}_{1}, \bar{T}_{2}\right] \\
\left(L_{\bar{T}_{1}+\bar{T}_{2}} u_{1}\right) \Leftarrow e=1 \kappa_{0} \Uparrow_{q_{1}}^{t_{1}} \varnothing \\
\left(R_{\bar{T}_{1}+\bar{T}_{2}} u_{2}\right) \Leftarrow e=f \kappa_{0} \Uparrow_{q_{2}}^{t_{2}}\left\{\kappa_{2}\right\} \\
p \Leftarrow e_{2}\left[u_{2} / y\right]=\kappa_{2} \Uparrow_{q_{2}^{\prime}}^{t_{2}^{\prime}} \kappa_{b}^{?} \\
\frac{\text { M-Case-2 } \quad}{p \Leftarrow \text { case } e^{\bar{T}_{1}+\bar{T}_{2}} \text { of }\left(L x \rightarrow e_{1}\right)\left(R y \rightarrow e_{2}\right)=\kappa \Uparrow_{q_{1} * q_{2} * q_{2}^{\prime}}^{t_{1} \cdot t_{2} \cdot t_{2}^{\prime}} \kappa_{b}^{?}}
\end{array} \\
& \left(\kappa_{0},\left[u_{1}, u_{2}\right]\right)=\text { fresh } \kappa\left[\bar{T}_{1}, \bar{T}_{2}\right] \\
& \left(L_{\bar{T}_{1}+\bar{T}_{2}} u_{1}\right) \Leftarrow e=\kappa_{0} \Uparrow_{q_{1}}^{t_{1}}\left\{\kappa_{1}\right\} \\
& \left(R_{\bar{T}_{1}+\bar{T}_{2}} u_{2}\right) \Leftarrow e=\kappa_{0} \Uparrow_{q_{2}}^{t_{2}} \varnothing \\
& \text { M-Case-3 } \quad p \Leftarrow e_{1}\left[u_{1} / x\right]=\kappa_{1} \Uparrow_{q_{1}^{\prime}}^{t_{1}^{\prime}} \kappa_{a}^{?} \\
& \overline{p \Leftarrow \text { case } e^{\bar{T}_{1}+\bar{T}_{2}} \text { of }\left(L x \rightarrow e_{1}\right)\left(R y \rightarrow e_{2}\right)=\kappa \Uparrow_{q_{1} * q_{2} * q_{1}^{\prime}}^{t_{1} \cdot t_{2} \cdot t_{1}^{\prime}} \kappa_{a}^{?}} \\
& \left(\kappa_{0},\left[u_{1}, u_{2}\right]\right)=\text { fresh } \kappa\left[\bar{T}_{1}, \bar{T}_{2}\right] \\
& \left(L_{\bar{T}_{1}+\bar{T}_{2}} u_{1}\right) \Leftarrow e=\kappa_{0} \Uparrow_{q_{1}}^{t_{1}} \varnothing \\
& \text { M-Case-4 }\left(R_{\bar{T}_{1}+\bar{T}_{2}} u_{2}\right) \Leftarrow e=\kappa_{0} \Uparrow_{q_{2}}^{t_{2}} \varnothing \\
& \overline{p \Leftarrow \text { case } e^{\bar{T}_{1}+\bar{T}_{2}} \text { of }\left(L x \rightarrow e_{1}\right)\left(R y \rightarrow e_{2}\right)=\kappa \Uparrow_{q_{1} * q_{2}}^{t_{1} \cdot t_{2}} \varnothing}
\end{aligned}
$$

Figure 15. Matching Semantics for Constraint-Solving case

where:

$$
\begin{aligned}
& l t=\operatorname{rec}(f: \text { nat } \rightarrow \text { nat } \rightarrow \text { bool }) x=\operatorname{rec}(g: \text { nat } \rightarrow \text { bool }) y= \\
& \text { case } y \text { of }\left(L_{-} \rightarrow \text { False }\right) \\
& \left(R y_{R} \rightarrow \text { case } x \text { of } \quad\left(L_{-} \rightarrow \text { True }\right)\right. \\
& \left.\left(R x_{R} \rightarrow f x_{R} y_{R}\right)\right)
\end{aligned}
$$

Many rules introduce fresh unknowns, many of which are irrelevant: they might be directly equivalent to some other unknown, or there might not exist any reference to them. We abusively use the same variable for two constraint sets which differ only in the addition of a few irrelevant variables to one of them.

Evaluation of $A \quad$ We first derive True $\Leftarrow(0<u)=\kappa \Uparrow_{1}^{\epsilon}\left\{\kappa_{0}\right\}$. Since in the desugaring of $0<u$ as an application $l t$ is already in rec form and both 0 and $u$ are values, the constraint set after the narrowing calls of M-App will stay unchanged. We then evaluate case $u$ of $\left(L_{-} \rightarrow\right.$ False $)\left(R y_{R} \rightarrow \ldots\right)$. Since the domain of $u$ contains both zero and non-zero elements, unifying $u$ with $L_{1+n a t} u_{1}$ and $R_{1+\text { nat }} u_{2}$ (M-Base) will produce some non-empty constraint sets. Therefore, rule M-Case-1 applies. Since the body of the left hand side of the match is False, the result of the left derivation in M-Case-1 is $\varnothing$ and in the resulting constraint set $\kappa_{0}$ the domain of $u$ is $\{1, \ldots, 9\}$.

Next, we turn to True $\Leftarrow(0<u \& \& u<4) \Rightarrow \kappa \Uparrow_{1}^{\epsilon}$ $\left\{\kappa_{1}\right\}$, where, by a similar argument following the recursion, the domain of $u$ in $\kappa_{1}$ is $\{1,2,3\}$. There are 3 possible narrowingsemantics derivations for ! $u$ : (1) $! u=\kappa_{1} \Downarrow_{1 / 3}^{[(0,3)]} \kappa_{1}^{A} \vDash u,(2)$ $! u=\kappa_{1} \Downarrow_{1 / 3}^{[(1,3)]} \kappa_{2}^{A} \models u$, and (3) $! u=\kappa_{1} \Downarrow_{1 / 3}^{[(2,3)]} \kappa_{3}^{A} \models u$, where the domain of $u$ in $\kappa_{i}^{A}$ is $\{i\}$. (We have switched to narrowingsemantics judgments because of the rule M-After.) Therefore all the possible derivations for $A=(0<u \& \& u<4)$; ! u matching True in $\kappa$ are:

$$
\text { True } \Leftarrow A=\kappa \Uparrow_{1 / 3}^{[(i-1,3)]}\left\{\kappa_{i}^{A}\right\} \quad \text { for } i \in\{1,2,3\}
$$

From the set of possible derivations, we can extract a probability distribution: for each resulting optional constraint set, we sum the probabilities of each of the traces that lead to this result. Thus the probability distribution associated with True $\Leftarrow A \Rightarrow \kappa$ is

$$
\left[\left\{\kappa_{1}^{A}\right\} \mapsto \frac{1}{3} ; \quad\left\{\kappa_{2}^{A}\right\} \mapsto \frac{1}{3} ; \quad\left\{\kappa_{3}^{A}\right\} \mapsto \frac{1}{3}\right]
$$

Evaluation of $B$ The evaluation of $0<u$ is the same as before, after which we narrow $! u$ directly in $\kappa_{0}$ and there are 9 possibilities: $! u=\kappa_{0} \Downarrow_{1 / 9}^{[(i-1,9)]} \kappa_{i}^{B} \models u$ for each $i \in\{1, \ldots, 9\}$, where the domain of $u$ in $\kappa_{i}^{B}$ is $\{i\}$. Then we evaluate True $\Leftarrow u<4 \Rightarrow \kappa_{i}^{B}$ : if $i$ is 1,2 or 3 this yields $\left\{\kappa_{i}^{B}\right\}$; if $i>3$ this yields a failure $\varnothing$. Therefore the possible derivations for $B=(0<u ; ! u) \& \& u<4$ are:

$$
\begin{array}{lr}
\text { True } \Leftarrow B=\kappa \Uparrow_{1 / 9}^{[(i-1,9)]}\left\{\kappa_{i}^{B}\right\} & \text { for } i \in\{1,2,3\} \\
\text { True } \Leftarrow B=\kappa \Uparrow_{1 / 9}^{[(i-1,9)]} \varnothing & \text { for } i \in\{4, \ldots, 9\}
\end{array}
$$

We can again compute the corresponding probability distribution:

$$
\left[\left\{\kappa_{1}^{B}\right\} \mapsto \frac{1}{9} ; \quad\left\{\kappa_{2}^{B}\right\} \mapsto \frac{1}{9} ; \quad\left\{\kappa_{3}^{B}\right\} \mapsto \frac{1}{9} ; \quad \varnothing \mapsto \frac{2}{3}\right]
$$

Note that if we were just recording the probability of an execution and not its trace, we would not know that there are six distinct executions leading to $\varnothing$ with probability $\frac{1}{9}$, so we would not be able to compute its total probability.

The probability associated with $\varnothing(0$ for $A, 2 / 3$ for $B)$ is the probability of backtracking. As stressed in $\$ 2$, $A$ is much better than $B$ in terms of backtracking-i.e., it is more efficient in this case to instantiate $u$ only after all the constraints on its domain have been recorded. For a more formal treatment of backtracking strategies in Luck using Markov Chains, see [28].

\subsection{Properties}

We close our discussion of Core Luck by formally stating and proving some key properties. Intuitively, we show that, when we evaluate an expression $e$ against a pattern $p$ in the presence of a constraint set $\kappa$, we can only remove valuations from the denotation of $\kappa$ (decreasingness), any derivation in the generator semantics corresponds to an execution in the predicate semantics (soundness), and every valuation that matches $p$ will be found in the denotation of the resulting constraint set of some derivation (completeness).

Since we have two flavors of generator semantics, narrowing and matching, we also present these properties in two steps. First, we present the properties for the narrowing semantics; their proofs have been verified using Coq-we just sketch them here. Then we present the properties for the matching semantics; for these, we have only paper proofs, but these proofs are quite similar to the narrowing ones (the only real difference is the case rule).

We begin by giving the formal specification of constraint setsand a few helpful lemmas that derive from it. We introduce one extra abstraction, the domain of a constraint set $\kappa$, written $\operatorname{dom}(\kappa)$. 
This domain corresponds to the unknowns in a constraint set that actually have bindings in $\llbracket \kappa \rrbracket$. For example, when we generate a fresh unknown $u$ from $\kappa, u$ does not appear in the domain of $\kappa$; it only appears in the denotation after we use it in a unification. The domain of $\kappa$ is a subset of the set of keys of $U(\kappa)$.

When we write that for a valuation and constraint set $\sigma \in \llbracket \kappa \rrbracket$, it also implies that the unknowns that have bindings in $\sigma$ are exactly the unknowns that have bindings in $\llbracket \kappa \rrbracket$, i.e., in $\operatorname{dom}(\kappa)$. We use the overloaded notation $\left.\sigma\right|_{x}$ to denote the restriction of $\sigma$ to $x$, where $x$ is either a set of unknowns or another valuation. The following straightforward lemma relates the two restrictions: ${ }^{2}$ if we restrict a valuation $\sigma^{\prime}$ to the domain of a constraint set $\kappa$, the resulting valuation is equivalent to restricting $\sigma^{\prime}$ to any valuation $\sigma \in \llbracket \kappa \rrbracket$.

Lemma 3.6.1. $\left.\left.\sigma \in \kappa \Rightarrow \sigma^{\prime}\right|_{\operatorname{dom}(\kappa)} \equiv \sigma^{\prime}\right|_{\sigma}$

Ordering We introduce an ordering on constraint sets: two constraints sets are ordered $\left(\kappa_{1} \leqslant \kappa_{2}\right)$ if $\operatorname{dom}\left(\kappa_{2}\right) \subseteq \operatorname{dom}\left(\kappa_{1}\right)$ and for all valuations $\sigma \in \llbracket \kappa_{1} \rrbracket,\left.\sigma\right|_{\operatorname{dom}\left(\kappa_{2}\right)} \in \llbracket \kappa_{2} \rrbracket$. Right away we can prove that $\leqslant$ is reflexive and transitive, using Lemma 3.6.1 and basic set properties.

\section{Specification of fresh}

$$
\left(\kappa^{\prime}, u\right)=\text { fresh } \kappa T \Rightarrow\left\{\begin{array}{l}
u \notin U(\kappa) \\
U\left(\kappa^{\prime}\right)=U(\kappa) \oplus(u \mapsto T) \\
\llbracket \kappa^{\prime} \rrbracket=\llbracket \kappa \rrbracket
\end{array}\right.
$$

Intuitively, when we generate a fresh unknown $u$ of type $T$ from $\kappa$, $u$ is really fresh for $\kappa$, meaning $U(\kappa)$ does not have a type binding for it. The resulting constraint set $\kappa^{\prime}$ has an extended unknown typing map, where $u$ maps to $T$ and its denotation remains unchanged. That means that $\operatorname{dom}\left(\kappa^{\prime}\right)=\operatorname{dom}(\kappa)$. Based on this specification, we can easily prove that $\kappa^{\prime}$ is smaller than $\kappa$, the generated unknowns are not contained in any valuation in $\llbracket \kappa \rrbracket$ and that $\kappa^{\prime}$ is well typed.

Lemma 3.6.2 (fresh_ordered).

$$
\left(\kappa^{\prime}, u\right)=\text { fresh } \kappa T \Rightarrow \kappa^{\prime} \leqslant \kappa
$$

Lemma 3.6.3 (fresh_for_valuation).

$$
\left(\kappa^{\prime}, u\right)=\text { fresh } \kappa T \Rightarrow \forall \sigma . \sigma \in \llbracket \kappa \rrbracket \Rightarrow u \notin \sigma
$$

Lemma 3.6.4 (fresh_types).

$$
\left(\kappa^{\prime}, u\right)=\text { fresh } \kappa T \Rightarrow\left(\vdash \kappa \Rightarrow \vdash \kappa^{\prime}\right)
$$

\section{Specification of sample}

$$
\kappa^{\prime} \in \text { sample } \kappa u \Rightarrow\left\{\begin{array}{l}
U\left(\kappa^{\prime}\right)=U(\kappa) \\
S A T\left(\kappa^{\prime}\right) \\
\exists v \cdot \llbracket \kappa^{\prime} \rrbracket=\{\sigma \mid \sigma \in \llbracket \kappa \rrbracket, \sigma(u)=v\}
\end{array}\right.
$$

When we sample $u$ in a constraint set $\kappa$ and obtain a list, for every member constraint set $\kappa^{\prime}$, the typing map of $\kappa$ remains unchanged and all of the valuations that remain in the denotation of $\kappa^{\prime}$ are the ones that mapped to some specific value $v$ in $\kappa$. Clearly, the domain of $\kappa$ remains unchanged. We also require a completeness property from sample, namely that if we have a valuation $\sigma \in \llbracket \kappa \rrbracket$ where $\sigma(u)=v$ for some $u, v$, then $\sigma$ is in some member $\kappa^{\prime}$ of the result:

$$
\left.\begin{array}{l}
\sigma(u)=v \\
\sigma \in \llbracket \kappa \rrbracket
\end{array}\right\} \Rightarrow \exists \kappa^{\prime} \cdot\left\{\begin{array}{l}
\sigma \in \llbracket \kappa^{\prime} \rrbracket \\
\kappa^{\prime} \in \text { sample } \kappa u
\end{array}\right.
$$

We can prove similar lemmas as in fresh: ordering and preservation.

In addition, we can show that if some unknown is a singleton in $\kappa$, it remains a singleton in $\kappa^{\prime}$. This is necessary for the proof of narrowing expressions.

\footnotetext{
${ }^{2}$ All the definitions in this section are implicitly universally quantified over the free variables appearing the formulas.
}

Lemma 3.6.5 (sample_ordered).

$$
\kappa^{\prime} \in \text { sample } \kappa u \Rightarrow \kappa^{\prime} \leqslant \kappa
$$

Lemma 3.6.6 (sample_types).

$$
\kappa^{\prime} \in \text { sample } \kappa u \Rightarrow\left(\vdash \kappa \Rightarrow \vdash \kappa^{\prime}\right)
$$

Lemma 3.6.7 (sample_preserves_singleton).

$$
\kappa\left[u^{\prime}\right] \neq \varnothing \wedge \kappa^{\prime} \in \text { sample } \kappa u \Rightarrow \kappa^{\prime}\left[u^{\prime}\right]=\kappa\left[u^{\prime}\right]
$$

Finally, we can lift all of these properties to sample $V$ by simple induction, using this spec to discharge the base case.

\section{Specification of unify}

$$
\begin{aligned}
& U\left(\text { unify } \kappa v_{1} v_{2}\right)=U(\kappa) \\
& \llbracket \text { unify } \kappa v_{1} v_{2} \rrbracket=\left\{\sigma \in \llbracket \kappa \rrbracket \mid \sigma\left(v_{1}\right)=\sigma\left(v_{2}\right)\right\}
\end{aligned}
$$

When we unify in a constraint set $\kappa$ two (well-typed for $\kappa$ ) values $v_{1}$ and $v_{2}$, the typing map remains unchanged while the denotation of the result contains just the valuations from $\kappa$ that when substituted into $v_{1}$ and $v_{2}$ make them equal. The domain of $\kappa^{\prime}$ is the union of the domain of $\kappa$ and the unknowns in $v_{1}, v_{2}$.

Once again, we can prove ordering and typing lemmas.

Lemma 3.6.8 (unify_ordered).

$$
\text { unify } \kappa v_{1} v_{2} \leqslant \kappa
$$

Lemma 3.6.9 (unify_types).

$$
\left.\begin{array}{l}
\varnothing ; U(\kappa) \vdash v_{1}: \bar{T} \\
\varnothing ; U(\kappa) \vdash v_{2}: \bar{T} \\
\vdash \kappa
\end{array}\right\} \Rightarrow \vdash \text { unify } \kappa v_{1} v_{2}
$$

Properties of the Narrowing Semantics With the above specification of constraint sets, we can proceed to proving our main theorems for the narrowing semantics: decreasingness, soundness and completeness. The first theorem, decreasingness states that we never add new valuations to our constraint sets; our semantics can only refine the denotation of the input $\kappa$.

Theorem 3.6.10 (Decreasingness).

$$
e=\kappa \Downarrow_{q}^{t} \kappa^{\prime} \models v \Rightarrow \kappa^{\prime} \leqslant \kappa
$$

Proof: By induction on the derivation of narrowing, using the lemmas about ordering for fresh (Lemma 3.6.2), sample (Lemma 3.6.5 and unify Lemma 3.6.8, followed by repeated applications of the transitivity of $\leqslant$.

Before we reach the other main theorems we need to prove preservation for the narrowing semantics; to do that we first need to prove that the typing map of constraint sets only increases when narrowing.

Lemma 3.6.11 (Narrowing Effect on Type Environments).

$$
e=\left.\kappa \Downarrow_{q}^{t} \kappa^{\prime} \models v \Rightarrow U\left(\kappa^{\prime}\right)\right|_{U(\kappa)} \equiv U(\kappa)
$$

Proof: By induction on the derivation.

Case $N$-Base: $\left.U(\kappa)\right|_{U(\kappa)} \equiv U(\kappa)$ by the definition of restriction. Case N-Pair: By the inductive hypothesis we have $\left.U\left(\kappa_{1}\right)\right|_{U(\kappa)} \equiv U(\kappa)$ and $\left.U\left(\kappa_{2}\right)\right|_{U\left(\kappa_{1}\right)} \equiv U\left(\kappa_{1}\right)$. The result follows by transitivity.

Case N-CasePair-P: Similar to N-Pair.

Case N-CasePair-U: By the inductive hypothesis we have $\left.U\left(\kappa_{a}\right)\right|_{U(\kappa)} \equiv U(\kappa)$ and $\left.U\left(\kappa^{\prime}\right)\right|_{U\left(\kappa_{c}\right)} \equiv U\left(\kappa_{c}\right)$. By transitivity, we only need to show that $\left.U\left(\kappa_{c}\right)\right|_{U\left(\kappa_{a}\right)} \equiv U\left(\kappa_{a}\right)$. This follows by transitivity of restrict (through $U\left(\kappa_{b}\right)$ ), and the specifications of fresh and unify.

Cases N-L, N-R: The induction hypothesis gives us $\left.U\left(\kappa^{\prime}\right)\right|_{U(\kappa)} \equiv U(\kappa)$, which is exactly what we want to prove. 
Cases N-Case-L, N-Case-R, N-App: Similar to N-CasePair-P. Cases $\mathbf{N}$-Case-U-*: For each of the four cases derived by inlining choose, we proceed exactly like N-CasePair-U.

Cases N-Narrow-*: For each of the four cases derived by inlining choose the result follows as in $\mathbf{N}$-CasePair-U, with additional uses of transitivity to accommodate the narrowing derivations for $e_{1}$ and $e_{2}$.

Case N-Bang: Directly from the induction hypothesis, as in N-L.

We also need to prove a form of context invariance for unknowns: we can substitute a typing map $U$ with a supermap $U^{\prime}$ in a typing relation.

Lemma 3.6.12 (Unknown Invariance).

$$
\left.\begin{array}{l}
\Gamma ; U \vdash e: T \\
\left.U^{\prime}\right|_{U} \equiv U
\end{array}\right\} \Rightarrow \Gamma ; U^{\prime} \vdash e: T
$$

Proof: By induction on the typing derivation for $e$. The only interesting case is the one regarding unknowns: we know for some unknown $u$ that $U(u)=T$ and that $\left.U^{\prime}\right|_{U} \equiv U$ and want to prove that $\Gamma ; U\left(U^{\prime}\right) \vdash u: T$. By the $\mathbf{T}-\mathbf{U}$ rule we just need to show that $U^{\prime}(u)=T$, which follows the definition of $\equiv$ and $\mid$. for maps.

We can now prove preservation: if a constraint set $\kappa$ is well typed and an expression $e$ has type $T$ in $U(\kappa)$ and the empty context, then if we narrow $e=\kappa$ to obtain $\kappa^{\prime} \models v, \kappa^{\prime}$ will be well typed and $v$ will also have the same type $T$ in $U\left(\kappa^{\prime}\right)$.

Theorem 3.6.13 (Preservation).

$$
\left.\begin{array}{l}
e=\kappa \Downarrow_{q}^{t} \kappa^{\prime} \models v \\
U(\kappa) \vdash e: T \\
\vdash \kappa
\end{array}\right\} \Rightarrow\left\{\begin{array}{l}
U\left(\kappa^{\prime}\right) \vdash v: T \\
\vdash \kappa^{\prime}
\end{array}\right.
$$

Proof: Again, we proceed by induction on the narrowing derivation. Case $N$-Base: Since $v=e$ and $\kappa^{\prime}=\kappa$, the result follows immediately from the hypothesis.

Case N-Pair: We have

$$
e_{1}=\kappa \Downarrow_{q_{1}}^{t_{1}} \kappa_{1} \models v_{1} \text { and } e_{2}=\kappa_{1} \Downarrow_{q_{2}}^{t_{2}} \kappa_{2} \models v_{2} .
$$

The inductive hypothesis for the first derivation gives us that

$$
\left.\forall T^{\prime} . \begin{array}{l}
U(\kappa) \vdash e_{1}: T^{\prime} \\
\vdash \kappa
\end{array}\right\} \Rightarrow\left\{\begin{array}{l}
U\left(\kappa_{1}\right) \vdash v_{1}: T^{\prime} \\
\vdash \kappa_{1}
\end{array} .\right.
$$

Similarly, the second inductive hypothesis gives us that

$$
\left.\forall T^{\prime} . \begin{array}{l}
U\left(\kappa_{1}\right) \vdash e_{2}: T^{\prime} \\
\vdash \kappa_{1}
\end{array}\right\} \Rightarrow\left\{\begin{array}{l}
U\left(\kappa_{2}\right) \vdash v_{2}: T^{\prime} \\
\vdash \kappa_{2}
\end{array} .\right.
$$

The typing assumption of the theorem states that

$$
U(\kappa) \vdash\left(e_{1}, e_{2}\right): T .
$$

We want to show that

$$
U\left(\kappa_{2}\right) \vdash\left(v_{1}, v_{2}\right): T \text { and } \vdash \kappa_{2} .
$$

By inversion on the typing relation for $\left(e_{1}, e_{2}\right)$ we know that there exist $T_{1}, T_{2}$ such that

$$
T=T_{1} \times T_{2} \text { and } U(\kappa) \vdash e_{1}: T_{1} \text { and } U(\kappa) \vdash e_{2}: T_{2} \text {. }
$$

We first instantiate the first inductive hypothesis on $T_{1}$ which gives us $U\left(\kappa_{1}\right) \vdash v_{1}: T_{1}$ and $\vdash \kappa_{1}$. Then, to instantiate the second one on $T_{2}$ and obtain $U\left(\kappa_{2}\right) \vdash v_{2}: T_{2}$ and $\vdash \kappa_{2}$, we need to show that $U\left(\kappa_{1}\right) \vdash e_{2}: T_{2}$, which follows by combining Lemma 3.6.11 and Unknown Invariance Lemma 3.6.12). The same combination also gives us $U\left(\kappa_{2}\right) \vdash e_{1}: T_{1}$. The result follows by the T-Pair constructor.

Case N-CasePair-P: We have

$$
e=\kappa \Downarrow_{q}^{t} \kappa_{a} \models\left(v_{1}, v_{2}\right) \text { and } e^{\prime}\left[v_{1} / x, v_{2} / y\right]=\kappa_{a} \Downarrow_{q^{\prime}}^{t^{\prime}} \kappa^{\prime} \models v \text {. }
$$

By the inductive hypothesis,

$$
\left.\forall T^{\prime} . \begin{array}{l}
U(\kappa) \vdash e: T^{\prime} \\
\vdash \kappa
\end{array}\right\} \Rightarrow\left\{\begin{array}{l}
U\left(\kappa_{a}\right) \vdash\left(v_{1}, v_{2}\right): T^{\prime} \\
\vdash \kappa_{a}
\end{array}\right.
$$

and

$$
\left.\forall T^{\prime} . \begin{array}{l}
U\left(\kappa_{a}\right) \vdash e^{\prime}\left[v_{1} / x, v_{2} / y\right]: T^{\prime} \\
\vdash \kappa_{a}
\end{array}\right\} \Rightarrow\left\{\begin{array}{l}
U\left(\kappa^{\prime}\right) \vdash v: T^{\prime} \\
\vdash \kappa^{\prime}
\end{array} .\right.
$$

The typing assumption gives us

$$
U(\kappa) \vdash \text { case e of }(x, y) \rightarrow e^{\prime}: T .
$$

Inversion on this typing relation means that there exist types $T_{1}, T_{2}$ such that

$$
U(\kappa) \vdash e: T_{1} \times T_{2} \text { and }\left(y \mapsto T_{2}, x \mapsto T_{1}\right) ; U(\kappa) \vdash e^{\prime}: T
$$

We want to prove that

$$
U\left(\kappa^{\prime}\right) \vdash v: T \text { and } \vdash \kappa^{\prime} .
$$

We instantiate the first inductive hypothesis on $T_{1} \times T_{2}$ and use inversion on the resulting typing judgment for $\left(v_{1}, v_{2}\right)$, which yields

$$
\vdash \kappa_{a} \text { and } U\left(\kappa_{a}\right) \vdash v_{1}: T_{1} \text { and } U\left(\kappa_{a}\right) \vdash v_{2}: T_{2} .
$$

By the second inductive hypothesis, we only need to show that

$$
\varnothing ; U\left(\kappa_{a}\right) \vdash e^{\prime}\left[v_{1} / x, v_{2} / y\right]: T \wedge \vdash \kappa_{a}
$$

Applying the Substitution Lemma twice, Unknown Invariance and Lemma 3.6.11 yields the desired result.

Case N-CasePair-U: Similarly to the previous case, we have

$$
e=\kappa \Downarrow_{q}^{t} \kappa_{a} \models u \text { and } e^{\prime}\left[u_{1} / x, u_{2} / y\right]=\kappa_{c} \Downarrow_{q^{\prime}}^{t^{\prime}} \kappa^{\prime} \models v,
$$

where $\left(\kappa_{b},\left[u_{1}, u_{2}\right]\right)=$ fresh $\kappa_{a}\left[\bar{T}_{1}, \bar{T}_{2}\right]$

and $\kappa_{c}=$ unify $\kappa_{b}\left(u_{1}, u_{2}\right) u$ and $U(\kappa) \vdash e: \bar{T}_{1} \times \bar{T}_{2}$.

As in the previous case we have two inductive hypotheses

$$
\left.\forall T^{\prime} . \begin{array}{l}
U(\kappa) \vdash e: T^{\prime} \\
\vdash \kappa
\end{array}\right\} \Rightarrow\left\{\begin{array}{l}
U\left(\kappa_{a}\right) \vdash u: T^{\prime} \\
\vdash \kappa_{a}
\end{array}\right.
$$

and

$$
\left.\forall T^{\prime} . \begin{array}{l}
U\left(\kappa_{c}\right) \vdash e^{\prime}\left[u_{1} / x, u_{2} / y\right]: T^{\prime} \\
\vdash \kappa_{c}
\end{array}\right\} \Rightarrow\left\{\begin{array}{l}
U\left(\kappa^{\prime}\right) \vdash v: T^{\prime} \\
\vdash \kappa^{\prime}
\end{array} .\right.
$$

The same typing assumption,

$$
U(\kappa) \vdash \text { case } e \text { of }(x, y) \rightarrow e^{\prime}: T,
$$

can be inverted to introduce $T_{1}$ and $T_{2}$ such that

$$
U(\kappa) \vdash e: T_{1} \times T_{2} \text { and }\left(y \mapsto T_{2}, x \mapsto T_{1}\right) ; U(\kappa) \vdash e^{\prime}: T \text {. }
$$

By type uniqueness, $\bar{T}_{1}=T_{1}$ and $\bar{T}_{2}=T_{2}$. Once again, we want to prove that

$$
U\left(\kappa^{\prime}\right) \vdash v: T \text { and } \vdash \kappa^{\prime} .
$$

Like in the previous case, by the first inductive hypothesis instantiated on $T_{1} \times T_{2}$ we get that $\kappa_{a}$ is well typed and $u$ has type $T_{1} \times T_{2}$ in $\kappa_{a}$. By the specification of fresh (Lemma 3.6.4. we get that $\kappa_{b}$ is well typed and that $U\left(\kappa_{b}\right)=U\left(\kappa_{a}\right) \oplus u_{1} \mapsto$ $T_{1} \oplus u_{2} \mapsto T_{2}$, where $u_{1}$ and $u_{2}$ do not appear in $U\left(\kappa_{a}\right)$. By the specification of unify we know that $U\left(\kappa_{c}\right)=U\left(\kappa_{b}\right)$ and Lemma 3.6.9 means that $\kappa_{c}$ is well typed. Finally, we instantiate the second inductive hypothesis on $T$, using the Substitution Lemma and Unknown Invariance to prove its premise.

Cases $N-L, N-R, N$-Fold: Follows directly from the induction hypothesis after inversion of the typing derivation for $e$.

Cases N-Case-L, N-Case-R, N-Unfold-F: Similar to N-CasePairP. 
Cases $\mathrm{N}$-Unfold-U, $\mathrm{N}$-Case-U-*: The unknown case for unfold as well as the four cases derived by inlining choose are similar to N-CasePair-U.

Case N-App: We have

$$
\begin{gathered}
e_{0}=\kappa \Downarrow_{q_{0}}^{t_{0}} \kappa_{a} \models v_{0}, \quad e_{1}=\kappa_{a} \Downarrow_{q_{1}}^{t_{1}} \kappa_{b} \models v_{1}, \\
e_{2}\left[v_{0} / f, v_{1} / x\right]=\kappa_{b} \Downarrow_{q_{2}}^{t_{2}} \kappa^{\prime} \models v,
\end{gathered}
$$

as well as the corresponding inductive hypotheses, where $v_{0}$ is of the form $\left(\operatorname{rec}\left(f: T_{1} \rightarrow T_{2}\right) x=e_{2}\right)$.

The preservation typing assumption states that $\kappa$ is well typed and that $U(\kappa) \vdash\left(e_{0} e_{1}\right): T_{2}^{\prime}$. Inverting this typing relation gives us that

$$
U(\kappa) \vdash e_{0}: T_{1}^{\prime} \rightarrow T_{2}^{\prime} \text { and } U(\kappa) \vdash e_{1}: T_{1}^{\prime} .
$$

By the first inductive hypothesis,

$$
\left.\forall T^{\prime} . \begin{array}{l}
U(\kappa) \vdash e_{0}: T^{\prime} \\
\vdash \kappa
\end{array}\right\} \Rightarrow\left\{\begin{array}{l}
U\left(\kappa_{a}\right) \vdash v_{0}: T^{\prime} \\
\vdash \kappa_{a}
\end{array} .\right.
$$

Instantiating this hypothesis on $T_{1}^{\prime} \rightarrow T_{2}^{\prime}$ and inverting the resulting typing relation gives us that $T_{1}^{\prime}=T_{1}$ and $T_{2}^{\prime}=T_{2}$. The remainder of the proof is similar to the second part of N-CasePair-P. Case N-Bang: We have

$$
e=\kappa \Downarrow_{q}^{t} \kappa_{a} \models v \text { and sample } V \kappa_{a} v \Rightarrow_{q^{\prime}}^{t^{\prime}} \kappa^{\prime} .
$$

By the inductive hypothesis,

$$
\left.\forall T^{\prime} . \begin{array}{l}
U(\kappa) \vdash e: T^{\prime} \\
\vdash \kappa
\end{array}\right\} \Rightarrow\left\{\begin{array}{l}
U\left(\kappa_{a}\right) \vdash v: T^{\prime} \\
\vdash \kappa_{a}
\end{array} .\right.
$$

The typing premise of preservation states that $e$ has type $\bar{T}$ in $U(\kappa)$, and we can instantiate the inductive hypothesis with $\bar{T}$. By the specification of sample, $U\left(\kappa^{\prime}\right)=U(\kappa)$ and the typing lemma for sample yields that $\kappa^{\prime}$ is well typed which concludes the proof. Case N-Inst-*:

Each of the four cases derived by inlining choose are similar. The typing premise is $\varnothing ; U(\kappa) \vdash e \leftarrow\left(e_{1}, e_{2}\right): T$, while we know that $U(\kappa) \vdash e: \bar{T}_{1} \times \bar{T}_{2}$. Inverting the premise and using type uniqueness allows us to equate $T$ with $\bar{T}_{1} \times \bar{T}_{2}$ and also gives us that $e_{1}$ and $e_{2}$ have type nat in $\kappa$.

We have that $e=\kappa \Downarrow_{q}^{t} \kappa_{a} \models v$ and the corresponding inductive hypothesis:

$$
\left.\forall T^{\prime} . \begin{array}{l}
U(\kappa) \vdash e: T^{\prime} \\
\vdash \kappa
\end{array}\right\} \Rightarrow\left\{\begin{array}{l}
U\left(\kappa_{a}\right) \vdash v: T^{\prime} \\
\vdash \kappa_{a}
\end{array} .\right.
$$

We instantiate it to $\bar{T}_{1} \times \bar{T}_{2}$. Using the narrowing of types and Unknown Invariance, we get that $e_{1}$ and $e_{2}$ have type nat in $\kappa_{a}$.

We proceed similarly for the derivations

$$
e_{1}=\kappa_{a} \Downarrow_{q_{1}}^{t_{1}} \kappa_{b} \models v_{1} \text { and } e_{2}=\kappa_{b} \Downarrow_{q_{2}}^{t_{2}} \kappa_{c} \models v_{2},
$$

using the induction hypothesis and propagating type information using Lemma 3.6.11 and Unknown Invariance, to obtain that $\kappa_{c}$ is well typed, $v$ has type $\bar{T}_{1} \times \bar{T}_{2}$, and $v_{1}$ and $v_{2}$ have type nat.

Continuing with the flow of the rule,

sample $V \kappa_{c} v_{1} \Rightarrow{ }_{q_{1}^{\prime}}^{t_{1}^{\prime}} \kappa_{d}$ and sample $V \kappa_{d} v_{2} \Rightarrow{ }_{q_{2}^{\prime}}^{t_{2}^{\prime}} \kappa_{e}$,

and the specification for sample lifted to sample $V$ yield $U\left(\kappa_{e}\right)=U\left(\kappa_{d}\right)=U\left(\kappa_{c}\right)$ and $\kappa_{e}$ is well typed.

We can then apply the specification of fresh to the generation of the unknowns $u_{1}$ and $u_{2}$,

$$
\left(\kappa_{0},\left[u_{1}, u_{2}\right]\right)=\text { fresh } \kappa_{e}\left[\bar{T}_{1}, \bar{T}_{2}\right],
$$

which means $U\left(\kappa_{0}\right)=U\left(\kappa_{e}\right) \oplus u_{1} \mapsto \bar{T}_{1} \oplus u_{2} \mapsto \bar{T}_{2}$ and $\vdash \kappa_{0}$. Therefore, $\left.U\left(\kappa_{0}\right)\right|_{U\left(\kappa_{e}\right)} \equiv U\left(\kappa_{e}\right)$ and by Unknown Invariance the type of $v$ carries over to $U\left(\kappa_{0}\right)$. In addition, $L_{\bar{T}_{1}+\bar{T}_{2}} u_{1}$ and $R_{\bar{T}_{1}+\bar{T}_{2}} u_{2}$ have the same type as $v$ in $\kappa_{0}$.
The two unifications operate on $\kappa_{0}$,

$$
\kappa_{l}=\text { unify } \kappa_{0} v\left(L_{\bar{T}_{1}+\bar{T}_{2}} u_{1}\right)
$$

and

$$
\kappa_{r}=\text { unify } \kappa_{0} v\left(R_{\bar{T}_{1}+\bar{T}_{2}} u_{2}\right),
$$

and the specification of unify applies to give us that $U\left(\kappa_{0}\right)=U\left(\kappa_{l}\right)=U\left(\kappa_{r}\right)$, as well as $\vdash \kappa_{l}$ and $\vdash \kappa_{r}$. Thus, for both $\kappa_{l}$ and $\kappa_{r}, v$ has type $\bar{T}_{1} \times \bar{T}_{2}$. Since choose will pick one of $\kappa_{l}$ or $\kappa_{r}$ to return, this concludes the proof.

With preservation for the narrowing semantics proved, we only need one very simple lemma about the interaction between variable and valuation substitution in expressions:

Lemma 3.6.14 (Substitution Interaction).

$$
\left.\begin{array}{l}
\sigma(e)=e^{\prime} \\
\sigma(v)=v^{\prime}
\end{array}\right\} \Rightarrow \sigma(e[v / x])=e^{\prime}\left[v^{\prime} / x\right]
$$

Proof: The result follows by induction on $\sigma(e)=e^{\prime}$ and case splitting on whether $x$ is equal to any variable encountered.

Soundness and completeness can be visualized as follows:

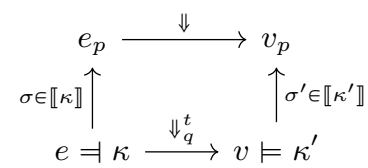

Given the bottom and right sides of the diagram, soundness guarantees that we can fill in the top and left. That is, any narrowing derivation $e=\kappa \Downarrow_{t}^{q} \kappa^{\prime} \models v$ directly corresponds to some derivation in the predicate semantics, with the additional assumption that all the unknowns in $e$ are included in the domain of the input constraint set $\kappa$ (which can be replaced by a stronger assumption that $e$ is well typed in $\kappa$ ).

Before we formally state and prove soundness, we need two technical lemmas about unknown inclusion in domains.

Lemma 3.6.15 (Narrow Result Domain Inclusion).

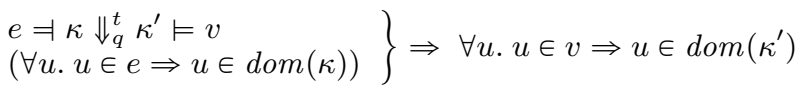

Proof: Straightforward induction on the narrowing derivation.

Lemma 3.6.16 (Narrow Increases Domain).

$$
\left.\begin{array}{l}
\begin{array}{l}
e=\kappa \Downarrow_{q}^{t} \kappa^{\prime} \models v \\
u \in \operatorname{dom}(\kappa)
\end{array}
\end{array}\right\} \Rightarrow u \in \operatorname{dom}\left(\kappa^{\prime}\right)
$$

Proof: Straightforward induction on the narrowing derivation, using the specifications of fresh, unify and sample.

We also need a sort of inverse to Substitution Interaction:

Lemma 3.6.17 (Inverse Substitution Interaction).

$$
\left.\begin{array}{c}
\sigma\left(e_{2}\right)=e_{2}^{\prime} \\
\sigma\left(e_{1}\left[e_{2} / x\right]\right)=e_{1}^{\prime \prime}
\end{array}\right\} \Rightarrow \exists e_{1}^{\prime} \cdot \sigma\left(e_{1}\right)=e_{1}^{\prime}
$$

Proof: By induction on $e_{1}$, inversion of the substitution relation and case analysis on variable equality when necessary.

We can now move on to stating and proving soundness for the narrowing semantics.

Theorem 3.6.18 (Soundness).

$$
\left.\begin{array}{l}
e=\kappa \Downarrow_{t}^{q} \kappa^{\prime} \models v \\
\sigma^{\prime}(v)=v_{p} \wedge \sigma^{\prime} \in \llbracket \kappa^{\prime} \rrbracket \\
\forall u . u \in e \Rightarrow u \in \operatorname{dom}(\kappa)
\end{array}\right\} \Rightarrow \exists \sigma e_{p} \cdot\left\{\begin{array}{l}
\left.\sigma^{\prime}\right|_{\sigma} \equiv \sigma \\
\sigma \in \llbracket \kappa \rrbracket \\
\sigma(e)=e_{p} \\
e_{p} \Downarrow v_{p}
\end{array}\right.
$$


Proof: By induction on the narrowing derivation.

Case N-Base: In the base case $e=v$ and therefore the soundness witnesses are trivially $\sigma^{\prime}$ and $v_{p}$.

Case N-Pair: We know that

$$
e_{1}=\kappa \Downarrow_{q_{1}}^{t_{1}} \kappa_{1} \models v_{1} \text { and } e_{2}=\kappa_{1} \Downarrow_{q_{2}}^{t_{2}} \kappa^{\prime} \models v_{2} .
$$

By inversion of the substitution $\sigma^{\prime}(v)$ we know that $v=\left(v_{1}, v_{2}\right)$, $\sigma\left(v_{1}\right)=v_{p_{1}}$ and $\sigma\left(v_{2}\right)=v_{p_{2}}$.

By the induction hypothesis for $e_{p_{2}}$, we get that

$$
\left.\begin{array}{l}
\sigma^{\prime}\left(v_{2}\right)=v_{p_{2}} \wedge \sigma^{\prime} \in \llbracket \kappa^{\prime} \rrbracket \\
\forall u . u \in e_{2} \Rightarrow u \in \operatorname{dom}\left(\kappa_{1}\right)
\end{array}\right\} \Rightarrow \exists \sigma_{1} e_{p_{2}} \cdot\left\{\begin{array}{l}
\left.\sigma^{\prime}\right|_{\sigma_{1}} \equiv \sigma_{1} \\
\sigma_{1} \in \llbracket \kappa_{1} \rrbracket \\
e_{p_{2}} \Downarrow v_{p_{2}} \\
\sigma_{1}\left(e_{2}\right)=e_{p_{2}}
\end{array} .\right.
$$

Its only premise that is not an assumption can be discharged using the lemma about narrowing increasing domain (Lemma 3.6.16.

The induction hypothesis for $e_{p_{1}}$ states that

$$
\left.\begin{array}{l}
\sigma_{1}\left(v_{1}\right)=v_{p_{1}} \wedge \sigma_{1} \in \llbracket \kappa_{1} \rrbracket \\
\forall u . u \in e_{1} \Rightarrow u \in \operatorname{dom}(\kappa)
\end{array}\right\} \Rightarrow \exists \sigma e_{p_{1}} \cdot\left\{\begin{array}{l}
\left.\sigma_{1}\right|_{\sigma} \equiv \sigma \\
\sigma \in \llbracket \kappa \rrbracket \\
e_{p_{1} \Downarrow v_{p_{1}}} \\
\sigma\left(e_{1}\right)=e_{p_{1}}
\end{array} .\right.
$$

Proving that $\sigma_{1}\left(v_{1}\right)=v_{p_{1}}$ is where the requirement that unknowns be bound in the input $\kappa$ comes into play: since $\sigma_{1}$ is a restriction of $\sigma^{\prime}$, they assign the same values to all unknowns that $\sigma_{1}$ assigns a value to. Using Lemma 3.6.15 we can show that all unknowns in $v_{1}$ are included in the domain of $\kappa_{1}$ and therefore $\sigma_{1}\left(v_{1}\right)=$ $\sigma^{\prime}\left(v_{1}\right)=v_{p_{1}}$.

The final witnesses for the $\mathbf{N}$-Pair case are $\sigma$ and $\left(e_{p_{1}}, e_{p_{2}}\right)$. The conclusion follows using the transitivity of restrict and the $\mathbf{P}$ Pair constructor.

\section{Case N-CasePair-P: We know that}

$$
e=\kappa \Downarrow_{q}^{t} \kappa_{a} \models\left(v_{1}, v_{2}\right) \text { and } e^{\prime \prime}=\kappa_{a} \Downarrow_{q^{\prime}}^{t^{\prime}} \kappa^{\prime} \models v,
$$

where $e^{\prime \prime}=e^{\prime}\left[v_{1} / x, v_{2} / y\right]$. The inductive hypothesis for the second derivation gives us

$$
\left.\begin{array}{l}
\sigma^{\prime}(v)=v_{p} \wedge \sigma^{\prime} \in \llbracket \kappa^{\prime} \rrbracket \\
\forall u . u \in e^{\prime \prime} \Rightarrow u \in \operatorname{dom}\left(\kappa_{a}\right)
\end{array}\right\} \Rightarrow \exists \sigma_{1} e_{p}^{\prime \prime} .\left\{\begin{array}{l}
\left.\sigma^{\prime}\right|_{\sigma_{1}} \equiv \sigma_{1} \\
\sigma_{1} \in \llbracket \kappa_{a} \rrbracket \\
e_{p}^{\prime \prime} \Downarrow v_{p} \\
\sigma_{1}\left(e^{\prime \prime}\right)=e_{p}^{\prime \prime}
\end{array} .\right.
$$

After discharging the premises using Lemma 3.6.15 and Lemma 3.6.16 we can investigate the shape of the $e_{p}^{\prime \prime}$ witness. First note that, because of the domain inclusions, there exist $v_{p_{1}}, v_{p_{2}}$ such that $\sigma_{1}\left(v_{1}\right)=v_{p_{1}}$ and $\sigma_{1}\left(v_{2}\right)=v_{p_{2}}$. But then, by applying Inverse Substitution Interaction Lemma 3.6.17) we know that there exists $e_{p}^{\prime}$ such that $\sigma_{1}\left(e^{\prime}\right)=e_{p}$.

By the inductive hypothesis for $e$, we get that

$$
\left.\begin{array}{l}
\sigma_{1}\left(\left(v_{1}, v_{2}\right)\right)=\left(v_{p_{1}}, v_{p_{2}}\right) \\
\sigma_{1} \in \llbracket \kappa_{a} \rrbracket \\
\forall u . u \in e \Rightarrow u \in \operatorname{dom}(\kappa)
\end{array}\right\} \Rightarrow \exists \sigma e_{p} \cdot\left\{\begin{array}{l}
\left.\sigma_{1}\right|_{\sigma} \equiv \sigma \\
\sigma \in \llbracket \kappa \rrbracket \\
e_{p} \Downarrow\left(v_{p_{1}}, v_{p_{2}}\right) \\
\sigma(e)=e_{p}
\end{array} .\right.
$$

This allows us to provide witnesses for the entire case: $\sigma$ and case $e_{p}$ of $(x, y) \rightarrow e_{p}^{\prime}$. Using the transitivity of restrict and the $\mathbf{P}$ CasePair rule, we only need to show that $e_{p}^{\prime \prime}=e_{p}^{\prime}\left[v_{p_{1}} / x, v_{p_{2}} / y\right]$, which follows from two applications of the (normal) Substitution Interaction lemma (Lemma 3.6.14.

Case N-CasePair-U: This case is largely similar with N-CasePair$\mathbf{P}$, with added details for dealing with fresh and unify. We have

$$
e=\kappa \Downarrow_{q}^{t} \kappa_{a} \models u^{\prime} \text { and } e^{\prime \prime}=\kappa_{c} \Downarrow_{q^{\prime}}^{t^{\prime}} \kappa^{\prime} \models v,
$$

where

$$
\begin{gathered}
e^{\prime \prime}=e^{\prime}\left[u_{1} / x, u_{2} / y\right], \\
\left(\kappa_{b},\left[u_{1}, u_{2}\right]\right)=\text { fresh } \kappa_{a}\left[\bar{T}_{1}, \bar{T}_{2}\right], \\
\kappa_{c}=\text { unify } \kappa_{b}\left(u_{1}, u_{2}\right) u^{\prime} .
\end{gathered}
$$

By the inductive hypothesis for the second derivation, we get

$$
\left.\begin{array}{l}
\sigma^{\prime}(v)=v_{p} \wedge \sigma^{\prime} \in \llbracket \kappa^{\prime} \rrbracket \\
\forall u . u \in e^{\prime \prime} \Rightarrow u \in \operatorname{dom}\left(\kappa_{c}\right)
\end{array}\right\} \Rightarrow \exists \sigma_{1} e_{p}^{\prime \prime} .\left\{\begin{array}{l}
\left.\sigma^{\prime}\right|_{\sigma_{1}} \equiv \sigma_{1} \\
\sigma_{1} \in \llbracket \kappa_{c} \rrbracket \\
e_{p}^{\prime \prime} \Downarrow v_{p} \\
\sigma_{1}\left(e^{\prime \prime}\right)=e_{p}^{\prime \prime}
\end{array} .\right.
$$

Discharging the inclusion premise is slightly less trivial in this case, since it requires using the specifications of fresh and unify to hand the case where $u=u_{1}$ or $u=u_{2}$.

Then, by the definition of $\kappa_{c}$, we know that $\sigma_{1}$ is in the denotation of unify $\kappa_{b}\left(u_{1}, u_{2}\right) u^{\prime}$. But, by the specification of unify, $\left.\sigma_{1}\right|_{\operatorname{dom}\left(\kappa_{b}\right)} \in \llbracket \kappa_{b} \rrbracket$. Since fresh preserves the domains of constraints sets, that also means that $\left.\sigma_{1}\right|_{\operatorname{dom}\left(\kappa_{a}\right)} \in \llbracket \kappa_{a} \rrbracket$. In the following, let $\sigma_{1}^{\prime}=\left.\sigma_{1}\right|_{\operatorname{dom}\left(\kappa_{a}\right)}$; then, since $u \in \operatorname{dom}\left(\kappa_{a}\right)$ by Lemma 3.6.15 there exists some value $v_{u}$ such that $\sigma_{1}^{\prime}(u)=v_{u}$.

We can now use the inductive hypothesis for the first narrowing derivation that states that:

$$
\left.\begin{array}{l}
\sigma_{1}^{\prime}(u)=v_{u} \wedge \sigma_{1}^{\prime} \in \llbracket \kappa_{a} \rrbracket \\
\forall u . u \in e \Rightarrow u \in \operatorname{dom}(\kappa)
\end{array}\right\} \Rightarrow \exists \sigma e_{p} \cdot\left\{\begin{array}{l}
\left.\sigma_{1}^{\prime}\right|_{\sigma} \equiv \sigma \\
\sigma \in \llbracket \kappa \rrbracket \\
e_{p} \Downarrow v_{u} \\
\sigma(e)=e_{p}
\end{array} .\right.
$$

We conclude as in N-CasePair-P, with $\sigma$ and case $e_{p}$ of $(x, y) \rightarrow$ $e_{p}^{\prime}$ as the witnesses, where $e_{p}^{\prime}$ is obtained as in the previous case by investigating the shape of $e_{p}^{\prime \prime}$.

Cases $\mathbf{N}-\mathbf{L}, \mathrm{N}$-R, $\mathrm{N}$-Fold: These cases follow similarly to $\mathrm{N}$-Pair. Cases $N$-Case-L, $N$-Case-R, $N$-Unfold-F, $N$-After: These cases follow similarly to N-CasePair-P.

Cases $\mathbf{N}$-Case-U, $\mathrm{N}$-Unfold-U: These cases follow similarly to $\mathbf{N}$ CasePair-U.

Case N-App: We have

$$
\begin{gathered}
e_{0}=\kappa \Downarrow_{q}^{t} \kappa_{a} \models v_{0}, \quad e_{1}=\kappa_{a} \Downarrow_{q}^{t} \kappa_{b} \models v_{1}, \\
e_{2}^{\prime}=\kappa_{b} \Downarrow_{q^{\prime}}^{t^{\prime}} \kappa^{\prime} \models v,
\end{gathered}
$$

where $v_{0}$ is of the form $\left(\operatorname{rec}\left(f: T_{1} \rightarrow T_{2}\right) x=e_{2}\right)$ and $e_{2}^{\prime}=e_{2}\left[v_{0} / f, v_{1} / x\right]$.

By the inductive hypothesis for the third derivation we get that

$$
\left.\begin{array}{l}
\sigma^{\prime}(v)=v_{p} \wedge \sigma^{\prime} \in \llbracket \kappa^{\prime} \rrbracket \\
\forall u . u \in e_{2}^{\prime} \Rightarrow u \in \operatorname{dom}\left(\kappa_{b}\right)
\end{array}\right\} \Rightarrow \exists \sigma_{2} e_{p_{2}}^{\prime} \cdot\left\{\begin{array}{l}
\left.\sigma^{\prime}\right|_{\sigma_{2}} \equiv \sigma_{2} \\
\sigma_{2} \in \llbracket \kappa_{b} \rrbracket \\
e_{p_{2}}^{\prime} \Downarrow v_{p} \\
\sigma_{2}\left(e_{2}^{\prime}\right)=e_{p_{2}}^{\prime}
\end{array} .\right.
$$

As in N-CasePair-P, we can prove that there exists $v_{p_{0}}$ and $v_{p_{1}}$ such that $\sigma_{2}\left(v_{0}\right)=v_{p_{0}}$ and $\sigma_{2}\left(v_{1}\right)=v_{p_{1}}$.

By the inductive hypothesis for the evaluation of the argument we get that there exist $\sigma_{1}$ and $e_{p_{1}}$ such that $\left.\sigma_{2}\right|_{\sigma_{1}} \equiv \sigma_{1}$ and $\sigma_{1} \in \llbracket \kappa_{a} \rrbracket$ and $\sigma\left(e_{1}\right)=e_{p_{1}}$ and $e_{p_{1}} \Downarrow v_{p_{1}}$.

Since $\sigma_{1}$ is a restriction of $\sigma_{2}$ and because of the inclusion hypotheses, $\sigma_{1}$ also maps the lambda to $v_{p_{0}}$, which allows us to use the last inductive hypothesis:

$$
\left.\begin{array}{l}
\sigma_{1}\left(v_{0}\right)=v_{p_{0}} \wedge \sigma_{1} \in \llbracket \kappa_{a} \rrbracket \\
\forall u . u \in e_{0} \Rightarrow u \in \operatorname{dom}(\kappa)
\end{array}\right\} \Rightarrow \exists \sigma e_{p_{0}} \cdot\left\{\begin{array}{l}
\left.\sigma_{1}\right|_{\sigma} \equiv \sigma \\
\sigma \in \llbracket \kappa \rrbracket \\
e_{p_{0}} \Downarrow v_{p_{0}} \\
\sigma\left(e_{0}\right)=e_{p_{0}}
\end{array} .\right.
$$

By inverting the substitution for $\sigma_{1}$ in the lambda expression, we know that there exists $e_{p_{2}}$, such that

$$
v_{p_{0}}=\left(\operatorname{rec}\left(f: T_{1} \rightarrow T_{2}\right) x=e_{p_{2}}\right) \text { and } \sigma_{1}\left(e_{2}\right)=e_{p_{2}} .
$$

The witnesses needed for soundness are $\sigma$ and $\left(e_{p_{0}} e_{p_{1}}\right)$. After using the transitivity of restrict and the P-App constructor, the only 
goal left to prove is that:

$$
e_{p_{2}}\left[v_{p_{0}} / f, v_{p_{1}} / x\right] \Downarrow v_{p} .
$$

Applying Substitution Interaction twice concludes the proof. Case N-Bang: We know that

$$
e=\kappa \Downarrow_{q}^{t} \kappa_{a} \models v \text { and sample } V \kappa_{a} v \Rightarrow_{q^{\prime}}^{t^{\prime}} \kappa^{\prime} .
$$

By the specification of sample, since $\sigma^{\prime} \in \llbracket \kappa^{\prime} \rrbracket$ we know that $\sigma^{\prime} \in \llbracket \kappa_{a} \rrbracket$ and the result follows directly from the induction hypothesis.

Case N-Narrow: The 4 derived cases from inlining choose flow similarly, so without loss of generality let's assume that the first choose rule was used. We know a lot of things from the narrowing derivation:

$$
\begin{gathered}
e=\kappa \Downarrow_{q}^{t} \kappa_{a} \models v, \\
e_{1}=\kappa_{a} \Downarrow_{q_{1}}^{t_{1}} \kappa_{b} \models v_{1}, \quad e_{2}=\kappa_{b} \Downarrow_{q_{2}}^{t_{2}} \kappa_{c} \models v_{2}, \\
\text { sampleV } \kappa_{c} v_{1} \Rightarrow \Rightarrow_{q_{1}^{\prime}}^{t^{\prime}} \kappa_{d}, \quad \text { sampleV } \kappa_{d} v_{2} \Rightarrow{ }_{q_{2}^{\prime}}^{t^{\prime}} \kappa_{e}, \\
\text { nat }_{\kappa_{e}}\left(v_{1}\right)=n_{1}, \quad n_{1}>0, \quad \text { nat }_{\kappa_{e}}\left(v_{2}\right)=n_{2}, \quad n_{2}>0, \\
\left(\kappa_{0},\left[u_{1}, u_{2}\right]\right)=\text { fresh } \kappa_{e}\left[\bar{T}_{1}, \bar{T}_{2}\right], \\
\kappa_{l}=\text { unify } \kappa_{0} v\left(L_{\bar{T}_{1}+\bar{T}_{2}} u_{1}\right), \\
\kappa_{r}=\text { unify } \kappa_{0} v\left(R_{\bar{T}_{1}+\bar{T}_{2}} u_{2}\right) .
\end{gathered}
$$

By the specification of unify for the definition of $\kappa_{l}$ we know that $\left.\sigma^{\prime}\right|_{\operatorname{dom}\left(\kappa_{0}\right)} \in \llbracket \kappa_{0} \rrbracket$. By using the specification of fresh we can

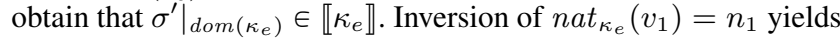
that there exists $v_{p_{1}}$ such that $\kappa_{e}\left[v_{1}\right]=v_{p_{1}}$ (where we lift the $\kappa[\cdot]$ notation to values) and similarly for $v_{p_{2}}$ (using Lemma 3.6.7 to preserve the first result). But that means, for all $\sigma \in \| \kappa_{e} \rrbracket$ (including $\left.\left.\sigma^{\prime}\right|_{\operatorname{dom}\left(\kappa_{e}\right)}\right)$, we have $\sigma\left(v_{i}\right)=v_{p_{i}}$.

That allows us to use the inductive hypotheses for $e_{1}$ and $e_{2}$ yielding $\sigma_{1}, e_{p_{1}}$ and $e_{p_{2}}$ such that $\left.\sigma^{\prime}\right|_{\sigma_{1}} \equiv \sigma_{1}, \sigma_{1}\left(e_{i}\right)=e_{p_{i}}$ and $e_{p_{i}} \Downarrow v_{p_{i}}$.

Finally, we use the last inductive hypothesis to obtain $\sigma$ and $e_{p}$ as appropriate and provide $\sigma$ and $e_{p} \leftarrow\left(e_{p_{1}}, e_{p_{2}}\right)$ as witnesses to the entire case. The result follows immediately.

Completeness guarantees the opposite direction: given a predicate derivation $e_{p} \Downarrow v_{p}$ and a "factoring" of $e_{p}$ into an expression $e$ and a constraint set $\kappa$ such that for some valuation $\sigma \in \llbracket \kappa \rrbracket$ substituting $\sigma$ in $e$ yields $e_{p}$, and under the assumption that everything is well typed, there is always a nonzero probability of obtaining some factoring of $v_{p}$ as the result of a narrowing judgment.

Theorem 3.6.19 (Completeness).

$$
\left.\begin{array}{l}
e_{p} \Downarrow v_{p} \\
\sigma(e)=e_{p} \\
\sigma \in \llbracket \kappa \rrbracket \wedge \vdash \kappa \\
\varnothing ; U(\kappa) \vdash e: T
\end{array}\right\} \Rightarrow\left\{\begin{array}{l}
\left.\sigma^{\prime}\right|_{\sigma} \equiv \sigma \wedge \sigma^{\prime} \in \llbracket \kappa^{\prime} \rrbracket \\
\sigma^{\prime}(v)=v_{p} \\
e=\kappa \Downarrow_{q}^{t} \kappa^{\prime} \models v
\end{array}\right.
$$

Proof: By induction on the derivation of the predicate semantics judgment.

Case P-Val: The witnesses for completeness are $v, \kappa, \sigma, 1$ and $\epsilon$. The result holds trivially.

Case P-Pair: We have

$$
e_{p_{1}} \Downarrow v_{p_{1}} \text { and } e_{p_{2}} \Downarrow v_{p_{2}} .
$$

By inversion on the substitution we have two cases. In the simple case, $e$ is some unknown $u$ and $\sigma(u)=\left(e_{p_{1}}, e_{p_{2}}\right)$. But then $e_{p_{1}}$ and $e_{p_{2}}$ must be values, and therefore $e_{p_{i}}=v_{p_{i}}$. By the N-Base rule, $u=\kappa \Downarrow_{1}^{\epsilon} \kappa \models u$ and the result follows directly.

In the more interesting case, $e$ is a pair $\left(e_{1}, e_{2}\right)$ and we know that $\sigma\left(e_{1}\right)=e_{p_{1}}$ and $\sigma\left(e_{2}\right)=e_{p_{2}}$. Inverting the typing relation gives us

$$
U(\kappa) \vdash e_{1}: T_{1} \text { and } U(\kappa) \vdash e_{2}: T_{2} .
$$

The inductive hypothesis for the first predicate semantics derivation, instantiated at $\sigma, \kappa$ and $T_{1}$ gives us that

$$
\left.\begin{array}{l}
\sigma \in \llbracket \kappa \rrbracket \\
\sigma\left(e_{1}\right)=e_{p_{1}} \\
\vdash \kappa \\
U(\kappa) \vdash e_{1}: T_{1}
\end{array}\right\} \Rightarrow\left\{\begin{array}{l}
\exists v_{1} \kappa_{1} \sigma_{1} q_{1} t_{1} . \\
e_{1}=\kappa \|_{\sigma} \wedge \sigma_{q_{1}} \wedge \kappa_{1} \models v_{1} \\
\sigma_{1}\left(v_{1}\right)=v_{p_{1}}
\end{array}\right.
$$

Its assumptions already hold so we can obtain such witnesses. By the second inductive hypothesis, we know that

$$
\left.\begin{array}{l}
\sigma_{1} \in \llbracket \kappa_{1} \rrbracket \\
\sigma_{1}\left(e_{1}\right)=e_{p_{1}} \\
\vdash \kappa_{1} \\
U\left(\kappa_{1}\right) \vdash e_{1}: T_{2}
\end{array}\right\} \Rightarrow\left\{\begin{array}{l}
\exists v_{2} \kappa_{2} \sigma_{2} q_{2} t_{2} . \\
\left.\sigma_{2}\right|_{\sigma_{1}} \equiv \sigma_{1} \wedge \sigma_{2} \in \llbracket \kappa_{2} \rrbracket \\
e_{1}=\kappa_{1} \Downarrow_{q_{2}}^{t_{2}} \kappa_{2} \models v_{1} \\
\sigma_{2}\left(v_{1}\right)=v_{p_{2}}
\end{array} .\right.
$$

Since $\left.\sigma_{1}\right|_{\sigma}$ and $\sigma\left(e_{1}\right)=e_{p_{1}}$, then $\sigma_{1}\left(e_{1}\right)=e_{p_{1}}$. By preservation, we get that $\kappa_{1}$ is well typed. Finally, narrowing only extends the typing environment Lemma 3.6.11 and then by Unknown Invariance (Lemma 3.6.12) we can obtain the last assumption $U(\kappa) \vdash e_{1}: T_{1}$ of the inductive hypothesis.

We combine the results from the two inductive hypotheses to provide witnesses for the existentials:

$$
\left(v_{1}, v_{2}\right), \quad \kappa_{2}, \sigma_{2}, q_{1} * q_{2} \text { and } t_{1} \cdot t_{2} .
$$

By transitivity of restrict, we get that $\left.\sigma \equiv \sigma_{2}\right|_{\sigma}$, while the inclusion property $\sigma_{2} \in \llbracket \kappa_{2} \rrbracket$ is satisfied by the inductive hypothesis directly. To prove that $\sigma_{2}\left(\left(v_{1}, v_{2}\right)\right)=\left(v_{p_{1}}, v_{p_{2}}\right)$ we just need to prove that $\sigma_{2}\left(v_{1}\right)=v_{p_{1}}$, but that holds because $\sigma\left(e_{1}\right)=e_{p_{1}}$ and $\sigma$ is a restriction of $\sigma_{2}$. Using the $\mathbf{N}$-Pair constructor completes the proof. Case P-App: We know that

$$
e_{p_{0}} \Downarrow v_{p_{0}}, e_{p_{1}} \Downarrow v_{p_{1}} \text { and } e_{p_{2}}\left[v_{p_{0}} / f, v_{p_{1}} / x\right] \Downarrow v_{p},
$$

for some $v_{p_{0}}=\left(\operatorname{rec}\left(f: T_{1} \rightarrow T_{2}\right) x=e_{p_{2}}\right)$. Inversion on the substitution gives us only one possible $e$, since unknowns only range over values: $e=\left(e_{0} e_{1}\right)$, where $\sigma\left(e_{0}\right)=e_{p_{0}}$ and $\sigma\left(e_{1}\right)=e_{p_{1}}$. Inversion of the typing premise gives us

$$
U(\kappa) \vdash e_{0}: T_{1}^{\prime} \rightarrow T_{2}^{\prime} \text { and } U(\kappa) \vdash e_{1}: T_{1}^{\prime} .
$$

By the inductive hypothesis for the derivation of $e_{p_{0}}$ we get that

$$
\left.\begin{array}{l}
\sigma \in \llbracket \kappa \rrbracket \\
\sigma\left(e_{0}\right)=e_{p_{0}} \\
\vdash \kappa \\
U(\kappa) \vdash e_{0}: T_{1}^{\prime} \rightarrow T_{2}^{\prime}
\end{array}\right\} \Rightarrow\left\{\begin{array}{l}
\left.\sigma_{0}\right|_{\sigma} \equiv \sigma \wedge \sigma_{0} \in \llbracket \kappa_{0} \rrbracket \\
e_{0}=\kappa \Downarrow_{q_{0}}^{t_{0}} \kappa_{0} \models v_{0} \\
\sigma_{0}\left(v_{0}\right)=v_{p_{0}}
\end{array}\right.
$$

All its assumptions hold, so we can invert the last substitution to obtain that $v_{0}=\left(\operatorname{rec}\left(f: T_{1} \rightarrow T_{2}\right) x=e_{2}\right)$, where $\sigma_{0}\left(e_{2}\right)=e_{p_{2}}$. By preservation, we know that the type of $v_{0}$ is the type of $e_{0}$ in $\kappa_{0}$ and uniqueness of typing equates $T_{1}$ with $T_{1}^{\prime}$ and $T_{2}$ with $T_{2}^{\prime}$.

By the second inductive hypothesis, we know that

$$
\left.\begin{array}{l}
\sigma_{0} \in \llbracket \kappa_{0} \rrbracket \\
\sigma_{0}\left(e_{1}\right)=e_{p_{1}} \\
\vdash \kappa_{0} \\
U\left(\kappa_{0}\right) \vdash e_{1}: T_{1}
\end{array}\right\} \Rightarrow\left\{\begin{array}{l}
\exists v_{1} \kappa_{1} \sigma_{1} q_{1} t_{1} . \\
e_{1}=\kappa_{0} \Downarrow_{0} \wedge \kappa_{q_{1}} \kappa_{1} \models v_{1} \\
\sigma_{1}\left(v_{1}\right)=v_{p_{1}}
\end{array} .\right.
$$

As in P-Pair we can discharge all of its assumptions.

Let $e_{2}^{\prime}=e_{2}\left[v_{0} / f, v_{1} / x\right]$ and $e_{p_{2}^{\prime}}=e_{p_{2}}\left[v_{p_{0}} / f, v_{p_{1}} / x\right]$. The last inductive hypothesis states that

$$
\left.\begin{array}{l}
\sigma_{1} \in \llbracket \kappa_{1} \rrbracket \\
\sigma_{1}\left(e_{2}^{\prime}\right)=e_{p_{2}^{\prime}} \\
\vdash \kappa_{1} \\
U\left(\kappa_{1}\right) \vdash e_{2}^{\prime}: T_{2}
\end{array}\right\} \Rightarrow\left\{\begin{array}{l}
\exists v_{2}^{\prime} \kappa_{2}^{\prime} \sigma_{2}^{\prime} q_{2}^{\prime} t_{2}^{\prime} . \\
e_{2}^{\prime}=\kappa_{1} \kappa_{1} \Downarrow_{q_{2}^{\prime}}^{t_{2}^{\prime}} \kappa_{2}^{\prime} \models v_{2}^{\prime} \\
\sigma_{2}^{\prime}\left(v_{2}^{\prime}\right)=v
\end{array}\right.
$$


The substitution premise can be discharged using the Substitution Interaction Lemma (Lemma 3.6.14, while the typing premise by repeated applications of the Substitution Lemma and Unknown Invariance. The proof concludes by combining the probabilities and traces by multiplication and concatenation respectively.

Cases P-L, P-R, P-Fold, P-After: These cases are similar to PPair.

Case P-CasePair: From the predicate derivations we have that

$$
e_{p} \Downarrow\left(v_{p_{1}}, v_{p_{2}}\right) \text { and } e_{p}^{\prime}\left[v_{p_{1}} / x, v_{p_{2}} / y\right] \Downarrow v_{p}^{\prime} .
$$

Inverting the substitution premise leaves us with $\sigma(e)=e_{p}$ and $\sigma\left(e^{\prime}\right)=e_{p}^{\prime}$, while inverting the typing premise yields

$$
U(\kappa) \vdash e: T_{1}+T_{2} \text { and }\left(x \mapsto T_{1}, y \mapsto T_{2}\right) ; U(\kappa) \vdash e^{\prime}: T .
$$

The inductive hypothesis for $e_{p}$ gives us that

$$
\left.\begin{array}{l}
\sigma \in \llbracket \kappa \rrbracket \\
\sigma(e)=e_{p} \\
\vdash \kappa \\
U(\kappa) \vdash e: T_{1}+T_{2}
\end{array}\right\} \Rightarrow\left\{\begin{array}{l}
\sigma_{1} \mid \sigma \equiv \sigma \wedge \sigma_{1} \in \llbracket \kappa_{1} \rrbracket \\
e=\kappa \Downarrow_{q_{1}}^{t_{1}} \kappa_{1} \models v \\
\sigma_{1}(v)=\left(v_{p_{1}}, v_{p_{2}}\right)
\end{array} .\right.
$$

To decide which of N-CasePair-P and $\mathbf{N}$-CasePair-U we will use, we invert the substitution relation for $v$. In the simple case, $v=\left(v_{1}, v_{2}\right)$ and the proof flows similarly to P-App.

The interesting case is when $v$ is an unknown $u$, in which case we need to "build up" the derivation of $\mathbf{N}$-CasePair-U. Let

$$
\begin{gathered}
\left(\kappa_{1 a},\left[u_{1}, u_{2}\right]\right)=\text { fresh } \kappa_{1}\left[T_{1}, T_{2}\right], \\
\kappa_{1 b}=\text { unify } \kappa_{1 a}\left(u_{1}, u_{2}\right) u, \\
\sigma_{1}^{\prime}=\sigma_{1} \oplus u_{1} \mapsto v_{1} \oplus u_{2} \mapsto v_{2}, \\
e^{\prime \prime}=e^{\prime}\left[u_{1} / x, u_{2} / y\right] \text { and } e_{p}^{\prime \prime}=e_{p}^{\prime}\left[v_{p_{1}} / x, v_{p_{2}} / y\right] .
\end{gathered}
$$

The second inductive hypothesis (instantiated at $\sigma_{1}^{\prime}, \kappa_{1 b}$ ) states that

$$
\left.\begin{array}{l}
\sigma_{1}^{\prime} \in \llbracket \kappa_{1 b} \rrbracket \\
\vdash \kappa_{1 b} \\
U\left(\kappa_{1 b}\right) \vdash e^{\prime \prime}: T \\
\sigma_{1}^{\prime}\left(e^{\prime \prime}\right)=e_{p}^{\prime \prime}
\end{array}\right\} \Rightarrow\left\{\begin{array}{l}
\left.\sigma_{2}\right|_{\sigma_{1}^{\prime}} \equiv \sigma_{1}^{\prime} \wedge \sigma_{2} \in \llbracket \kappa_{2} \rrbracket \\
\sigma\left(v^{\prime}\right)=v_{p}^{\prime} \\
e^{\prime \prime}=\kappa_{1 b} \Downarrow_{q_{2}}^{t_{2}} \kappa_{2} \models v^{\prime}
\end{array}\right.
$$

To use this inductive hypothesis we need to discharge all of its assumptions first.

To prove that $\sigma_{1}^{\prime} \in \llbracket \kappa_{1 b} \rrbracket$ we start with $\sigma_{1} \in \llbracket \kappa_{1} \rrbracket$ by the first induction hypothesis. By the specification of fresh, the denotation of $\kappa_{1}$ remains unchanged, therefore $\sigma_{1} \in \llbracket \kappa_{1 a} \rrbracket$. Since $u_{1}, u_{2}$ are not in the domain of $\kappa_{1 a}$, the restriction $\left.\sigma_{1}^{\prime}\right|_{\left.\text {dom( } \kappa_{1 a}\right)}$ is $\sigma_{1}$. Therefore, by the specification of unify we just need to show that $\sigma_{1}^{\prime}(u)=\sigma_{1}^{\prime}\left(\left(u_{1}, u_{2}\right)\right)$. Indeed,

$$
\begin{aligned}
\sigma_{1}^{\prime}(u)=\sigma_{1}(u)=\left(v_{p_{1}}, v_{p_{2}}\right) & =\left(\sigma_{1}^{\prime}\left(u_{1}\right), \sigma_{1}^{\prime}\left(u_{2}\right)\right) \\
& =\sigma_{1}^{\prime}\left(\left(u_{1}, u_{2}\right)\right),
\end{aligned}
$$

which concludes the proof of the first premise.

The fact that $\kappa_{1 b}$ is well typed is a direct corollary of the typing lemmas for fresh (Lemma 3.6.4) and unify (Lemma 3.6.9).

To prove that $U\left(\kappa_{1 b}\right) \vdash e^{\prime \prime}: T$ we apply the Substitution Lemma twice. Then we need to prove that

$$
\begin{gathered}
\varnothing ; U\left(\kappa_{1 b}\right) \vdash u_{i}: T_{i} \text { for } i=1,2 \\
\text { and }\left(x \mapsto T_{1}, y \mapsto T_{2}\right) ; U\left(\kappa_{1 b}\right) \vdash e^{\prime}: T .
\end{gathered}
$$

By the specification of unify we know that $U\left(\kappa_{1 b}\right)=U\left(\kappa_{1 a}\right)$, while from the specification of fresh we obtain

$$
U\left(\kappa_{1 a}\right)=U\left(\kappa_{1}\right) \oplus u_{1} \mapsto T_{1} \oplus u_{2} \mapsto T_{2} .
$$

This directly proves the former results for $u_{1}, u_{2}$, while Unknown Invariance (since $U\left(\kappa_{1 b}\right)$ is an extension of $U(\kappa)$ ) proves the latter.
The final premise of the inductive hypothesis, $\sigma_{1}^{\prime}\left(e^{\prime \prime}\right)=e_{p}^{\prime \prime}$, is easily proved by applying the Substitution Interaction lemma (Lemma 3.6.14) twice.

Since we have satisfied all of its premises, we can now use the result of the second inductive hypothesis. It provides most of the witnesses to completeness ( $v, \kappa_{2}$ and $\sigma_{2}$ ), while, as usual, we combine the probabilities and traces by multiplying and concatenating them. The result follows by transitivity of restrict and use of the N-CasePair-U constructor.

Cases P-Case-L, P-Case-R, P-Unfold: These cases are in direct correspondence with P-CasePair. The only difference is that to choose between which choose rule to follow we case analyze on the satisfiability of the corresponding constraint set.

Case P-Bang: We know that $e_{p} \Downarrow v_{p}$. By the inductive hypothesis, we immediately obtain that there exists some $v, \sigma_{1}, \kappa_{1}, q_{1}$ and $t_{1}$ such that $\sigma_{1}(v)=v_{p}$ and $\sigma_{1} \in \llbracket \kappa \rrbracket$ and $\left.\sigma_{1}\right|_{\sigma} \equiv \sigma$ and that $e=\kappa \Downarrow_{q_{1}}^{t_{1}} \kappa_{1} \models v$. By the completeness requirement of sample lifted to sample $V$, we know that there exists some $q_{2}, t_{2}$ and $\kappa_{2}$ such that sampleV $\kappa_{1} \quad v \Rightarrow_{q_{2}}^{t_{2}} \kappa_{2}$ and $\sigma_{1} \in \llbracket \kappa_{2} \rrbracket$. The result follows easily.

Case P-Narrow: We know that

$$
e_{p} \Downarrow v_{p}, \quad e_{p_{1}} \Downarrow v_{p_{1}} \text { and } e_{p_{2}} \Downarrow v_{p_{2}},
$$

while $\llbracket v_{p_{1}} \rrbracket>0$ and $\llbracket v_{p_{2}} \rrbracket>0$. Chaining the induction hypothesis as in P-Pair, we get that there exist $v, v_{1}, v_{2}, \sigma^{\prime}, \kappa_{1}, \kappa_{2}, \kappa^{\prime}, q, q_{1}$, $q_{2}, t, t_{1}$ and $t_{2}$ such that

$$
\begin{aligned}
& \left.\sigma^{\prime}\right|_{\sigma} \equiv \sigma \quad e=\kappa \Downarrow_{q}^{t} \kappa_{1} \models v \\
& \sigma^{\prime} \in \llbracket \kappa^{\prime} \rrbracket, \quad \sigma^{\prime}(v)=v_{p} \quad \text { and } \quad e_{1}=\kappa_{1} \Downarrow_{q_{1}}^{t_{1}} \kappa_{2} \models v_{1} \\
& \sigma^{\prime}\left(v_{i}\right)=v_{p_{i}} \quad e_{2}=\kappa_{2} \Downarrow_{q_{2}}^{t_{2}} \kappa_{3} \models v_{2}
\end{aligned}
$$

By the lifted completeness requirement of sample, we know that there exist $q_{1}^{\prime}, q_{2}^{\prime}, t_{1}^{\prime}, t_{2}^{\prime}, \kappa_{4}$ and $\kappa_{5}$ such that $\sigma \in \llbracket \kappa_{5} \rrbracket$,

$$
\text { sample } V \kappa_{3} v_{1} \Rightarrow{ }_{q_{1}^{\prime}}^{t_{1}^{\prime}} \kappa_{4} \text { and sampleV } \kappa_{4} v_{2} \Rightarrow{ }_{q_{2}^{\prime}}^{t_{2}^{\prime}} \kappa_{5} \text {. }
$$

By definition, $\operatorname{nat}_{\kappa_{5}}\left(v_{1}\right)=v_{p_{1}}$ and $n a t_{\kappa_{5}}\left(v_{2}\right)=v_{p_{2}}$.

Without loss of generality, assume that $v_{p}=L v_{p}^{\prime}$ for some $v_{p}^{\prime}$ and let

$$
\sigma^{\prime \prime}=\sigma^{\prime} \oplus u_{1} \mapsto v_{p}^{\prime} \text { and }\left(\kappa^{\prime},\left[u_{1}, u_{2}\right]\right)=\text { fresh } \kappa_{5}\left[T_{1}, T_{2}\right]
$$

and

$$
\kappa_{l}=\text { unify } \kappa^{\prime}\left(L u_{1}\right) v \text { and } \kappa_{r}=\text { unify } \kappa^{\prime}\left(R u_{2}\right) v \text {. }
$$

By transitivity of restrict, $\left.\sigma^{\prime \prime}\right|_{\sigma} \equiv \sigma$. Moreover, $\sigma^{\prime \prime}(v)=v_{p}$. The proof that $\sigma^{\prime \prime} \in \llbracket \kappa_{l} \rrbracket$ is similar to the proof that $\sigma_{1}^{\prime} \in \llbracket \kappa_{1 b} \rrbracket$ in P-Pair. To conclude the proof, we case analyze on whether $\kappa_{r}$ is satisfiable or not and choosing which choose derivation to follow accordingly.

Properties of the Matching Semantics Before we proceed to the theorems for the matching semantics, we need a specification for the union operation.

\section{Specification of union}

$$
\begin{gathered}
\left.U\left(\kappa_{1}\right)\right|_{U\left(\kappa_{1}\right) \cap U\left(\kappa_{2}\right)}=\left.U\left(\kappa_{2}\right)\right|_{U\left(\kappa_{1}\right) \cap U\left(\kappa_{2}\right)} \\
\text { union } \kappa_{1} \kappa_{2}=\kappa \\
\left\{\begin{array}{l}
U(\kappa)=U\left(\kappa_{1}\right) \cup U\left(\kappa_{2}\right) \\
\llbracket \kappa \rrbracket=\llbracket \kappa_{1} \rrbracket \cup \llbracket \kappa_{2} \rrbracket
\end{array}\right.
\end{gathered}
$$

To take the union of two constraint sets, their typing maps must obviously agree on any unknowns present in both. The denotation of the union of two constraint sets is then just the union of their corresponding denotations. 
Similar lemmas concerning types and ordering can be proved about union.

Lemma 3.6.20 (union_ordered).

$$
\kappa^{\prime}=\text { union } \kappa_{1} \kappa_{2} \Rightarrow \kappa^{\prime} \leqslant \kappa_{1} \wedge \kappa^{\prime} \leqslant \kappa_{2}
$$

Lemma 3.6.21 (union_types).

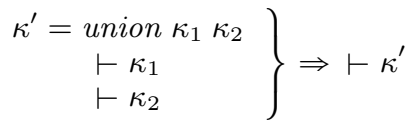

Specification of rename The rename function as introduced in the previous section can be encoded in terms of fresh and a function that renames a single unknown to the result of fresh, iteratively.

The decreasingness property for the matching semantics is very similar to the narrowing semantics: if the matching semantics yields $\left\{\kappa^{\prime}\right\}$, then $\kappa^{\prime}$ is smaller than the input constraint set.

Theorem 3.6.22 (Decreasingness).

$$
p \Leftarrow e=\kappa \Uparrow_{q}^{t}\left\{\kappa^{\prime}\right\} \Rightarrow \kappa^{\prime} \leqslant \kappa
$$

Proof: This is again the simplest proof: by induction on the derivation of matching judgment, using the lemmas about ordering for fresh Lemma 3.6.2), sample (Lemma 3.6.5) and unify Lemma 3.6.8 and repeated applications of the transitivity of $\leqslant$.

Preservation is simpler than before since we only deal with a single output. We still need a similar lemma about the effect of the matching semantics on types:

Lemma 3.6.23 (Matching Effect on Types).

$$
p \Leftarrow e=\left.\kappa \Uparrow_{q}^{t} v\left\{\kappa^{\prime}\right\} \Rightarrow U\left(\kappa^{\prime}\right)\right|_{U(\kappa)} \equiv U(\kappa)
$$

Proof: By induction on the derivation and transitivity of restrict.

Theorem 3.6.24 (Preservation).

$$
\left.\begin{array}{l}
p \Leftarrow e=\kappa \Uparrow_{q}^{t}\left\{\kappa^{\prime}\right\} \\
U(\kappa) \vdash e: \bar{T} \\
U(\kappa) \vdash p: \bar{T} \\
\vdash \kappa
\end{array}\right\} \Rightarrow \vdash \kappa^{\prime}
$$

Proof:

Case M-Base: Follows directly from the typing lemma of unify (Lemma 3.6.9).

Case M-Pair: We know that

$$
u_{1} \Leftarrow e_{1}=\kappa_{0} \Uparrow_{q_{1}}^{t_{1}}\left\{\kappa_{1}\right\} \text { and } u_{2} \Leftarrow e_{2}=\kappa_{1} \Uparrow_{q_{2}}^{t_{2}}\left\{\kappa_{2}\right\},
$$

where

$$
\begin{gathered}
\left(\kappa^{\prime},\left[u_{1}, u_{2}\right]\right)=\text { fresh } \kappa\left[\bar{T}_{1}, \bar{T}_{2}\right], \\
\kappa_{0}=\text { unify } \kappa^{\prime}\left(u_{1}, u_{2}\right) p .
\end{gathered}
$$

By inversion of the typing relation for $\left(e_{1}, e_{2}\right)$ we know that

$$
U(\kappa) \vdash e_{1}: \bar{T}_{1} \text { and } U(\kappa) \vdash e_{2}: \bar{T}_{2} .
$$

Based on the specification of fresh,

$$
U\left(\kappa^{\prime}\right)=U(\kappa) \oplus u_{1} \mapsto \bar{T}_{1} \oplus u_{2} \mapsto \bar{T}_{2} \text { and } \vdash \kappa^{\prime},
$$

while unify preserves all type information. Therefore, $\kappa_{0}$ is well typed and $U\left(\kappa_{0}\right) \vdash u_{1}: \bar{T}_{1}$ and $U\left(\kappa_{0}\right) \vdash u_{2}: \bar{T}_{2}$. By Unknown Invariance (Lemma 3.6.12) $e_{1}$ and $e_{2}$ are well typed in $\kappa_{0}$ as well.

Now we can use the inductive hypothesis for the derivation of $u_{1}$ which gives us that $\vdash \kappa_{1}$. To conclude the proof, we can use the other inductive hypothesis; for that we just need to show that $U\left(\kappa_{1}\right) \vdash u_{2}: \bar{T}_{2}$ and $U\left(\kappa_{1}\right) \vdash e_{2}: \bar{T}_{2}$. However, by the typing lemma for the matching semantics (Lemma 3.6.23) we known that $\left.U\left(\kappa_{1}\right)\right|_{U\left(\kappa_{0}\right)} \equiv U\left(\kappa_{0}\right)$. Unknown Invariance completes this case. Case M-CasePair: We know that

$$
\left(u_{1}, u_{2}\right) \Leftarrow e=\kappa_{a} \Uparrow_{q_{1}}^{t_{1}}\left\{\kappa_{b}\right\} \text { and } p \Leftarrow e^{\prime \prime}=\kappa_{b} \Uparrow_{q_{2}}^{t_{2}}\left\{\kappa^{\prime}\right\},
$$

where

$$
\begin{gathered}
e^{\prime \prime}=e^{\prime}\left[u_{1} / x, u_{2} / y\right], \\
\left(\kappa_{a},\left[u_{1}, u_{2}\right]\right)=\text { fresh } \kappa\left[\bar{T}_{1}, \bar{T}_{2}\right] .
\end{gathered}
$$

Like in the M-Pair case, using the definition of fresh we can obtain that $U\left(\kappa^{\prime}\right)=U(\kappa) \oplus u_{1} \mapsto \bar{T}_{1} \oplus u_{2} \mapsto \bar{T}_{2}$ as well as $\vdash \kappa_{a}$ and therefore $U\left(\kappa_{a}\right) \vdash\left(u_{1}, u_{2}\right): \bar{T}_{1} \times \bar{T} T_{2}$. We again can invert the typing relation for the entire case to obtain that

$$
U(\kappa) \vdash e: \bar{T}_{1}^{\prime} \times \bar{T}_{2}^{\prime} \text { and } x \mapsto \bar{T}_{1}^{\prime}, y \mapsto \bar{T}_{2}^{\prime} ; U(\kappa) \vdash e^{\prime}: \bar{T},
$$

while type uniqueness equates $\bar{T}_{i}$ with $\bar{T}_{i}^{\prime}$. Using Unknown Invariance we can propagate these typing relations to $\kappa_{a}$.

We can now use the inductive hypothesis on the matching derivation for $e$ to obtain that $\kappa_{b}$ is well typed. By the typing lemma for the matching semantics and Unknown Invariance we lift all typing relations to $\kappa_{b}$. To conclude the proof using the second inductive hypothesis we need only prove that $U\left(\kappa_{b}\right) \vdash e^{\prime}\left[u_{1} / x, u_{2} / y\right]: \bar{T}$, which follows by consecutive applications of the Substitution Lemma.

Cases M-L-Sat, M-R-Sat, M-Fold: Follow similarly to M-Pair. Case M-App: For some $v_{0}=\left(\operatorname{rec} f x=e_{2}\right)$, we have that

$$
e_{0}=\kappa \Downarrow_{q_{0}}^{t_{0}} \kappa_{0} \models v_{0} \text { and } e_{1}=\kappa_{0} \Downarrow_{q_{1}}^{t_{1}} \kappa_{1} \models v_{1},
$$

while

$$
p \Leftarrow e^{\prime}\left[v_{0} / f, v_{1} / x\right] \Rightarrow \kappa_{1} \Uparrow_{q_{2}}^{t_{2}}\left\{\kappa^{\prime}\right\} .
$$

By inverting the typing relation for $e_{0} e_{1}$ we get that $U(\kappa) \vdash$ $e_{0}: T \rightarrow \bar{T}$ and $U(\kappa) \vdash e_{1}: T$. Using the preservation theorem for the narrowing semantics Theorem 3.6.13 we know that $\kappa_{0}$ is well typed and the lambda has the same type as $e_{0}$ in $\kappa$. That means that

$$
(f \mapsto(T \rightarrow \bar{T}), x \mapsto T) ; U\left(\kappa_{0}\right) \vdash e_{2}: \bar{T} .
$$

The typing lemma for the narrowing semantics Lemma 3.6.11 and Unknown Invariance allow us to lift type information to $\kappa_{0}$. We repeat this process for the second narrowing derivation. To use the inductive hypothesis and conclude the proof, we only need to apply the Substitution Lemma twice as in M-CasePair.

Case M-Unfold: This case follows directly from the induction hypothesis.

Case M-After: We know that

$$
p \Leftarrow e_{1}=\kappa \Uparrow_{q_{1}}^{t_{1}}\left\{\kappa_{1}\right\} \text { and } e_{2}=\kappa_{1} \Downarrow_{q_{2}}^{t_{2}} \kappa_{2} \models v .
$$

As in M-Pair, we invert the typing relation to obtain type information for $e_{1}$ and $e_{2}$. We the use the inductive hypothesis on the first derivation to obtain that $\kappa_{1}$ is well typed. To conclude the proof, we need only apply the preservation lemma for the narrowing semantics, and its premise that $e_{2}$ is well typed is discharged as usual using the typing lemma for the matching semantics and Unknown Invariance.

Cases M-Pair-Fail, M-CasePair-Fail, M-Til-Fail, M-L-UnSat, MR-UnSat, M-Case-4, M-Bang-Fail, M-Narrow-Fail: These cases are vacuously true since no constraint set is returned.

Cases M-CasePair-Fun, M-Case-L-Fun, M-Case-R-Fun: Similar to M-App.

Case M-Bang: We know that

$$
p \Leftarrow e=\kappa \Uparrow_{q_{1}}^{t_{1}}\left\{\kappa_{1}\right\},
$$

where

$$
\text { sample } V \kappa_{1} p \Rightarrow_{q_{2}}^{t_{2}} \kappa^{\prime}
$$


By the inductive hypothesis we immediately get that $\kappa_{1}$ is well typed. The specification of sample lifted to sample $V$ yields the result.

Case M-Narrow: We know that

$$
p \Leftarrow e=\kappa \Uparrow_{q}^{t}\left\{\kappa_{a}\right\}
$$

and

$$
e_{1}=\kappa_{a} \Downarrow_{q_{1}}^{t_{1}} \kappa_{b} \models v_{1} \text { and } e_{2}=\kappa_{b} \Downarrow_{q_{2}}^{t_{2}} \kappa_{c} \models v_{2} .
$$

As in the previous cases, we use the inductive hypothesis and the preservation lemma for the narrowing semantics to ensure all variables are appropriately typed in $\kappa_{c}$.

Following the matching judgment, we proceed to sampleV twice resulting in a constraint set $\kappa_{e}$; as in M-Bang, $\kappa_{e}$ is well typed. We then generate two unknowns $u_{1}$ and $u_{2}$ with types $\bar{T}_{1}$ and $\bar{T}_{2}$ to obtain a constraint set $\kappa_{0}$, that is well typed because of the specification of fresh. Finally, we unify the pattern $p$ with the fresh unknowns tagged $L$ or $R$, yielding $\kappa_{l}$ and $\kappa_{r}$ that are both well typed because of the specification of unify. Since all choose does is pick which of $\kappa_{l}$ and $\kappa_{r}$ to return, the result follows immediately.

Cases M-Case-1, M-Case-2, M-Case-3: These cases flow similarly, using repeated applications of the inductive hypotheses. The only case that hasn't been encountered in a previous rule is for MCase-1, when both branch derivations yield some (well-typed) constraint sets $\left\{\kappa_{a}\right\}$ and $\left\{\kappa_{b}\right\}$ that are combined using union. But by the typing lemma for union, its result is also well typed.

Soundness is again similar to the matching semantics.

Theorem 3.6.25 (Soundness).

$$
\left.\begin{array}{l}
p \Leftarrow e=\kappa \Uparrow_{q}^{t}\left\{\kappa^{\prime}\right\} \\
\sigma^{\prime}(p)=v_{p} \wedge \sigma^{\prime} \in \llbracket \kappa^{\prime} \rrbracket \\
\forall u .(u \in e \vee u \in p) \Rightarrow u \in \operatorname{dom}(\kappa)
\end{array}\right\} \Rightarrow \exists \sigma e_{p} .\left\{\begin{array}{l}
\left.\sigma^{\prime}\right|_{\sigma} \equiv \sigma \\
\sigma \in \llbracket \kappa \rrbracket \\
\sigma(e)=e_{p} \\
e_{p} \Downarrow v_{p}
\end{array}\right.
$$

Proof: By induction on the matching derivation, following very closely the structure of proof of soundness for the narrowing semantics: we use the inductive hypothesis for every matching derivation in reverse order, obtaining witnesses for valuations and expressions, while concluding the proof with the specifications of constraint set operations and transitivity.

Case M-Base: In the base case, just like in the proof for the $\mathbf{N}$ Base rule, the witnesses are $\sigma^{\prime}$ and $v_{p}$. The inclusion $\sigma^{\prime} \in \kappa$ is a direct result of the specification of unify.

Case M-Pair: We know that

$$
u_{1} \Leftarrow e_{1} \Rightarrow \kappa_{0} \Uparrow_{q_{1}}^{t_{1}}\left\{\kappa_{1}\right\} \text { and } u_{2} \Leftarrow e_{2}=\kappa_{1} \Uparrow_{q_{2}}^{t_{2}}\left\{\kappa^{\prime}\right\},
$$

where

$$
\left(\kappa_{a},\left[u_{1}, u_{2}\right]\right)=\text { fresh } \kappa\left[\bar{T}_{1}, \bar{T}_{2}\right]
$$

and

$$
\kappa_{0}=\text { unify } \kappa_{a}\left(u_{1}, u_{2}\right) p .
$$

By the definition of fresh and the fact that the domain is increasing, we know that $u_{2}$ is in the domain of $\kappa^{\prime}$. That means that there exists some value $v_{p_{2}}^{\prime}$ such that $\sigma^{\prime}\left(u_{2}\right)=v_{p_{2}}^{\prime}$. By the inductive hypothesis for $\sigma^{\prime}$ and $u_{2}$ we get that there exist some $\sigma_{1}$ and $e_{p_{2}}$ such that $\sigma_{1}$ is a restriction of $\sigma^{\prime}$ in $\kappa_{1}$, while

$$
\sigma_{1}\left(e_{2}\right)=e_{p_{2}} \text { and } e_{p_{2}} \Downarrow v_{p_{2}}^{\prime} \text {. }
$$

Using a similar argument to obtain a $v_{p_{1}}^{\prime}$ such that $\sigma_{1}\left(u_{1}\right)=$ $v_{p_{1}}^{\prime}$, we can leverage the inductive hypothesis again on the first derivation gives us that there exists some $\sigma$ and $e_{p_{1}}$ such that $\sigma$ is a restriction of $\sigma_{1}$ in $\kappa_{0}$ and

$$
\sigma\left(e_{1}\right)=e_{p_{1}} \text { and } e_{p_{1}} \Downarrow v_{p_{1}}^{\prime} \text {. }
$$

Our soundness witnesses are $\sigma$ and $\left(e_{p_{1}}, e_{p_{2}}\right)$. By the specification of unify we know that $\sigma(p)=\sigma\left(\left(u_{1}, u_{2}\right)\right)$ and decreasingness helps us conclude that $v_{p}=\left(v_{p_{1}}^{\prime}, v_{p_{2}}^{\prime}\right)$ which concludes the proof of the pair case, along with transitivity of valuation restriction.

Cases M-Case-1, M-Case-2, M-Case-3: The only new rules are the case rules; however, the general structure of the proof is once again similar. For M-Case-1, we know that:

$$
\begin{gathered}
\left(\kappa_{0},\left[u_{1}, u_{2}\right]\right)=\text { fresh } \kappa\left[\bar{T}_{1}, \bar{T}_{2}\right], \\
\left(L_{\bar{T}_{1}+\bar{T}_{2}} u_{1}\right) \Leftarrow e=\kappa_{0} \Uparrow_{q_{1}}^{t_{1}}\left\{\kappa_{1}\right\}, \\
\left(R_{\bar{T}_{1}+\bar{T}_{2}} u_{2}\right) \Leftarrow e=\kappa_{0} \Uparrow_{q_{2}}^{t_{2}}\left\{\kappa_{2}\right\}, \\
p \Leftarrow e_{1}\left[u_{1} / x_{l}\right]=\kappa_{1} \Uparrow_{q_{1}^{\prime}}^{t_{1}^{\prime}} \kappa_{a}^{?} \text { and } p \Leftarrow e_{2}\left[u_{2} / y_{r}\right]=\kappa_{2} \Uparrow_{q_{2}^{\prime}}^{t_{2}^{\prime}} \kappa_{b}^{?},
\end{gathered}
$$

while

$$
\kappa^{?}=\text { combine } \kappa_{0} \kappa_{a}^{?} \kappa_{b}^{?} .
$$

If either of the non-union combine cases fire, the proof is simple. If $\kappa_{a}^{?}=\kappa_{b}^{?}=\varnothing$, then there exists no $\kappa^{\prime}$ such that the result of the derivation is $\left\{\kappa^{\prime}\right\}$.

Let's assume that $\kappa_{a}^{?}=\left\{\kappa_{a}\right\}$ for some $\kappa_{a}$ and $\kappa_{b}^{?}=\varnothing$ (the symmetric case follows similarly). Then we know that $\sigma^{\prime} \in \llbracket \kappa_{a} \rrbracket$ and from the inductive hypothesis for the $e_{1}$ derivation we get that there exist $\sigma_{1}$ and $e_{p_{1}}$ such that $\sigma \in \llbracket \kappa_{1} \rrbracket$ and $\sigma_{1}\left(e_{1}\left[u_{1} / x_{l}\right]\right)=$ $e_{p_{1}}$. As in the narrowing soundness proof, we can leverage the inverse substitution interaction lemma (3.6.14) to conclude that there exists some $e_{1}^{\prime}$ such that $\sigma_{1}\left(e_{1}\right)=e_{1}^{\prime}$. An additional application of the inductive hypothesis for the evaluation of $e$ against the $L_{\bar{T}_{1}+\bar{T}_{2}} u_{1}$ gives us $\sigma$ and $e_{p}$ such that $\sigma \in \llbracket \kappa \rrbracket$ and $\sigma(e)=e_{p}$, which are also the soundness witnesses that conclude the proof.

The more interesting case is when $\kappa_{a}^{?}=\left\{\kappa_{a}\right\}$ and $\kappa_{b}^{?}=\left\{\kappa_{b}\right\}$ for some constraint sets $\kappa_{a}$ and $\kappa_{b}$. In that case, $\sigma^{\prime} \in \llbracket \kappa_{a} \rrbracket$ or $\sigma^{\prime} \in \llbracket$ rename $\left(U\left(\kappa_{a}\right)-U\left(\kappa_{0}\right)\right) \kappa_{b} \rrbracket$. The first case proceeds exactly like the one for $\kappa_{b}^{?}=\varnothing$. For the latter, we need to push the renaming to $\sigma^{\prime}$, obtaining some $\sigma_{r}$ which is an alpha-converted version of $\sigma^{\prime}$ and then proceed similarly. Since the alpha conversion only happens in the unknowns that are not present in the original constraint set, the choice of these unknowns doesn't matter for the final witness.

For the completeness theorem, we need to slightly strengthen its premise; since the matching semantics may explore both branches of a case, it can fall into a loop when the predicate semantics would not (by exploring a non-terminating branch that the predicate semantics does not take). Thus, we require that all valuations in the input constraint set result in a terminating execution.

Before we go to completeness we need an auxiliary lemma that ensures there exists some derivation that returns a constraint set option if this requirement holds. This is only necessary for the combining M-Case-1.

Lemma 3.6.26 (Termination).

$$
\left.\begin{array}{l}
\varnothing ; U(\kappa) \vdash e: \bar{T} \wedge \vdash \kappa \\
\forall \sigma \in \llbracket \kappa \rrbracket . \exists v^{\prime} \cdot \sigma(e) \Downarrow v^{\prime}
\end{array}\right\} \Rightarrow \exists \kappa^{?} q t . p \Leftarrow e=\kappa \Uparrow_{q}^{t} \kappa^{?}
$$

The proof of this lemma is almost identical to the completeness proof. Since it doesn't require or enforce particular valuation memberships of the constraint sets involved, every case can follow with the same argument. The only rules where the difference matters is in the case rules, where the lack of assumptions allows to provide some termination witness without guaranteeing that the resulting constraint set is not $\varnothing$.

We also need another straightforward lemma regarding the completeness of values: 
Lemma 3.6.27 (Value Completeness).

$$
\left.\begin{array}{l}
U(\kappa) \vdash e: \bar{T} \\
\vdash \kappa \\
\sigma \in \llbracket \kappa \rrbracket \\
\sigma(e)=v_{p} \\
\sigma(p)=v_{p}
\end{array}\right\} \Rightarrow \exists \kappa^{\prime} \sigma^{\prime} q t .\left\{\begin{array}{l}
\left.\sigma^{\prime}\right|_{\sigma} \equiv \sigma \\
\sigma^{\prime} \in \llbracket \kappa^{\prime} \rrbracket \\
p \Leftarrow e=\mid \kappa \Uparrow_{q}^{t}\left\{\kappa^{\prime}\right\}
\end{array}\right.
$$

Proof: By induction on $e$.

Case $e=()$ or $e=u$ : If $e$ was unit or an unknown, let $\kappa^{\prime}=$ unify $\kappa$ e p. By the specification of unify $\sigma \in \llbracket \kappa^{\prime} \rrbracket$. The witnesses to conclude the case are $\kappa^{\prime}, \sigma, 1$ and $\epsilon$ using the M-Base rule.

Case $e=\left(e_{1}, e_{2}\right)$ : Following the M-Pair rule, let

$$
\left(\kappa^{\prime},\left[u_{1}, u_{2}\right]\right)=\operatorname{fresh} \kappa\left[\bar{T}_{1}, \bar{T}_{2}\right]
$$

and

$$
\kappa_{0}=\text { unify } \kappa^{\prime}\left(u_{1}, u_{2}\right) p .
$$

We invert the substitution relation to obtain that $\sigma\left(e_{1}\right)=v_{p_{1}}$ and $\sigma\left(e_{2}\right)=v_{p_{2}}$ for some $v_{p_{1}}, v_{p_{2}}$. Let $\sigma^{\prime}=\sigma \oplus u_{1} \mapsto v_{p_{1}} \oplus u_{2} \mapsto$ $v_{p_{2}}$. Then since $u_{1}$ and $u_{2}$ are fresh, $\left.\sigma^{\prime}\right|_{\sigma} \equiv \sigma$ and, by the specification of unify, $\sigma^{\prime} \in \kappa^{\prime}$.

By the inductive hypothesis for $e_{1}$ (inverting the typing relation for the typing premise), there exist $\sigma_{1}, \kappa_{1}, q_{1}$ and $t_{1}$ such that $\left.\sigma_{1}\right|_{\sigma^{\prime}} \equiv \sigma^{\prime}$ and $\sigma_{1} \in \llbracket \kappa_{1} \rrbracket$ and

$$
u_{1} \Leftarrow e_{1}=\kappa_{0} \Uparrow_{q_{1}}^{t_{1}} \kappa_{1} .
$$

Using Unknown Invariance we can apply the second inductive hypothesis to get similar $\sigma_{2}, \kappa_{2}, q_{2}$ and $t_{2}$. We conclude the case by providing the witnesses $\sigma_{2}$ and $\kappa_{2}$, while combining the probabilities and traces as usual $\left(q_{1} * q_{2}\right.$ and $\left.t_{1} \cdot t_{2}\right)$.

Cases $L, R$ or fold: The remaining cases are similar to the pair case, with only one inductive hypothesis.

Finally, we will need to propagate the termination information across matching derivations. For that we can prove the following corollary of decreasingness:

\section{Corollary 3.6.28. Termination Preservation}

$$
\left.\begin{array}{l}
p \Leftarrow e=\kappa \Uparrow_{q}^{t} \kappa^{\prime} \\
\forall \sigma \in \llbracket \kappa \rrbracket . \exists v \cdot \sigma(e) \Downarrow v
\end{array}\right\} \Rightarrow \forall \sigma^{\prime} \in \llbracket \kappa^{\prime} \rrbracket . \exists v \cdot \sigma^{\prime}(e) \Downarrow v
$$

Proof: By decreasingness, $\kappa^{\prime} \leqslant \kappa$, which means that $\left.\sigma^{\prime}\right|_{\sigma} \in \llbracket \kappa \rrbracket$. Then, there exists $v$ such that $\left.\sigma^{\prime}\right|_{\sigma}(e) \Downarrow v$ and the result follows.

\section{Theorem 3.6.29 (Completeness).}

$$
\left.\begin{array}{l}
e_{p} \Downarrow v_{p} \wedge \sigma \in \llbracket \kappa \rrbracket \\
\varnothing ; U(\kappa) \vdash e: \bar{T} \wedge \vdash \kappa \\
\sigma(e)=e_{p} \wedge \sigma(p)=v_{p} \\
\forall \sigma^{\prime} \in \llbracket \kappa \rrbracket . \exists v^{\prime} \cdot \sigma^{\prime}(e) \Downarrow v^{\prime}
\end{array}\right\} \Rightarrow \begin{aligned}
& \exists \kappa^{\prime} \sigma^{\prime} q t . \\
& \left\{\begin{array}{l}
\left.\sigma^{\prime}\right|_{\sigma} \equiv \sigma \\
\sigma^{\prime} \in \llbracket \kappa^{\prime} \rrbracket \\
p \Leftarrow e=\mid \kappa \Uparrow_{q}^{t}\left\{\kappa^{\prime}\right\}
\end{array}\right.
\end{aligned}
$$

Proof: By induction on the predicate derivation.

Case P-Val: Follows directly from the completeness lemma for values.

Case P-Pair: We have

$$
e_{p_{1}} \Downarrow v_{p_{1}} \text { and } e_{p_{2}} \Downarrow v_{p_{2}} .
$$

As in the narrowing proof, we invert the substitution of $e$ and get two cases. In the simple case, $e$ is some unknown $u$ and $\sigma(u)=$ $\left(e_{p_{1}}, e_{p_{2}}\right)$. But then $e_{p_{1}}$ and $e_{p_{2}}$ must be values, and the proof follows by the value completeness lemma.

In the more interesting case, $e$ is a pair $\left(e_{1}, e_{2}\right)$ and we know that $\sigma\left(e_{1}\right)=e_{p_{1}}$ and $\sigma\left(e_{2}\right)=e_{p_{2}}$. Inverting the typing relation gives us

$$
U(\kappa) \vdash e_{1}: \bar{T}_{1} \text { and } U(\kappa) \vdash e_{2}: \bar{T}_{2} .
$$

Following the M-Pair rule, let

$$
\left(\kappa^{\prime},\left[u_{1}, u_{2}\right]\right)=\text { fresh } \kappa\left[\bar{T}_{1}, \bar{T}_{2}\right]
$$

and

$$
\kappa_{0}=\text { unify } \kappa^{\prime}\left(u_{1}, u_{2}\right) p .
$$

As in the value completeness lemma, let $\sigma_{0}=\sigma \oplus u_{1} \mapsto v_{p_{1}} \oplus$ $u_{2} \mapsto v_{p_{2}}$. By the inductive hypothesis for the derivation of $e_{p_{1}}$,

$$
\begin{aligned}
& U\left(\kappa_{0}\right) \vdash e_{1}: \bar{T}_{1} \\
& \vdash \kappa_{0} \wedge \sigma_{0} \in \llbracket \kappa_{0} \rrbracket \\
& \sigma_{0}\left(e_{1}\right)=e_{p_{1}} \\
& \sigma_{0}\left(u_{1}\right)=v_{p_{1}} \\
& \forall \sigma^{\prime} \in \llbracket \kappa_{0} \rrbracket . \exists v^{\prime} . \sigma^{\prime}\left(e_{1}\right) \Downarrow v^{\prime} \\
& \begin{array}{l}
\exists \kappa_{1} \sigma_{1} q_{1} t_{1} . \\
\left\{\begin{array}{l}
\sigma_{1} \mid \sigma_{0} \equiv \sigma_{0} \\
\sigma_{1} \in \llbracket \kappa_{1} \rrbracket \\
u_{1} \Leftarrow e_{1}=\mid \kappa_{0} \Uparrow_{q_{1}}^{t_{1}}\left\{\kappa_{1}\right\}
\end{array}\right.
\end{array}
\end{aligned}
$$

Since $u_{1}$ and $u_{2}$ are fresh, we get that $\left.\sigma_{0}\right|_{\sigma} \equiv \sigma$ as well as $\sigma_{0}\left(\left(u_{1}, u_{2}\right)\right)=\sigma_{0}(p)$. But then by the specification of unify $\sigma_{0} \in \llbracket \kappa_{0} \rrbracket$. Moreover, by the ordering lemmas for fresh and unify (Lemma 3.6.2 and Lemma 3.6.8 we know that $\kappa_{0} \leqslant \kappa$ which means that the termination assumption for valuations in $\kappa$ is preserved and we can now use the above inductive hypothesis.

The inductive hypothesis for the derivation of $e_{p_{2}}$ yields

$$
\begin{aligned}
& U\left(\kappa_{1}\right) \vdash e_{2}: \bar{T}_{2} \\
& \vdash \kappa_{1} \wedge \sigma_{1} \in \llbracket \kappa_{1} \rrbracket \\
& \sigma_{1}\left(e_{2}\right)=e_{p_{2}} \\
& \begin{array}{l}
\sigma_{1}\left(u_{2}\right)=v_{p_{2}} \\
\forall \sigma^{\prime} \in \llbracket \kappa_{1} \rrbracket . \exists v^{\prime} . \sigma^{\prime}\left(e_{2}\right) \Downarrow v^{\prime}
\end{array}
\end{aligned}
$$

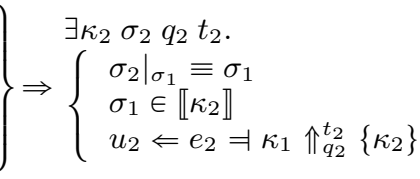

Like in the narrowing proof, we can discharge the typing hypothesis by using a lemma similar to Lemma 3.6.11 (which in turn is once again a simple induction on the matching derivation) and Unknown Invariance, while the termination assumption can be discharged using the Termination Preservation corollary.

Our final witnesses are $\sigma_{2}, \kappa_{2}$ and the standard combinations of probabilities and traces.

Case P-App: We know that

$$
e_{p_{0}} \Downarrow v_{p_{0}}, \quad e_{p_{1}} \Downarrow v_{p_{1}} \text { and } e_{p_{2}} \Downarrow v_{p},
$$

where $v_{p_{0}}$ is of the form $\left(\operatorname{rec}\left(f: T_{1} \rightarrow T_{2}\right) x=e_{p_{2}}\right)$ and $e_{p_{2}}^{\prime}=e_{p_{2}}\left[v_{p_{0}} / f, v_{p_{1}} / x\right]$. Inversion on the substitution gives us only one possible $e$, since unknowns only range over values: $e=\left(e_{0} e_{1}\right)$, where $\sigma\left(e_{0}\right)=e_{p_{0}}$ and $\sigma\left(e_{1}\right)=e_{p_{1}}$. Inversion of the typing premise gives us

$$
U(\kappa) \vdash e_{0}: T_{1}^{\prime} \rightarrow \bar{T}_{2}^{\prime} \text { and } U(\kappa) \vdash e_{1}: \bar{T}_{1}^{\prime} .
$$

Using preservation and type uniqueness we can equate $T_{1}$ with $T_{1}^{\prime}$ as well as $T_{2}$ with $\bar{T}_{2}^{\prime}$.

We can then turn to the completeness theorem for the narrowing semantics twice to obtain witnesses such that:

$$
e_{0}=\kappa \Downarrow_{q_{0}}^{t_{0}} \kappa_{0} \models\left(\operatorname{rec}\left(f: T_{1} \rightarrow \bar{T}_{2}\right) x=e_{2}\right)
$$

and

$$
e_{1}=\kappa_{0} \Downarrow_{q_{1}}^{t_{1}} \kappa^{\prime} \models v_{1} .
$$

Completeness also guarantees that there exists $\sigma^{\prime} \in \llbracket \kappa^{\prime} \rrbracket$ such that $\left.\sigma^{\prime}\right|_{\sigma} \equiv \sigma$, as well as $\sigma^{\prime}\left(e_{1}\right) \Downarrow \sigma^{\prime}\left(v_{1}\right)$ and, through restriction to $\operatorname{dom}\left(\kappa_{0}\right), \sigma^{\prime}\left(e_{0}\right) \Downarrow\left(\operatorname{rec}\left(f: T_{1} \rightarrow \bar{T}_{2}\right) x=\sigma\left(e_{2}\right)\right)$.

Using a Termination Preservation corollary for the narrowing semantics (that can be proved identically to the one for the matching semantics), in addition to Substitution Interaction as in the narrowing proof, we can use the inductive hypothesis for the substituted $e^{\prime}$ to complete the proof.

The rest of the cases follow using similar arguments, with the same overall structure as the narrowing proof. The only cases that are interestingly different (and where the termination assumption actually comes into play) are the ones that necessitate use of the 
combining case rule M-Case-1, which are P-Case-L and P-CaseR.

Case P-Case-L: Once again, the only interestingly different cases are the ones for the pattern matching constructs. For P-Case-L, we know that

$$
e_{p} \Downarrow L_{\bar{T}_{1}+\bar{T}_{2}} v_{p_{1}} \text { and } e_{p_{1}}\left[v_{p_{1}} / x\right] \Downarrow v_{p_{1}}^{\prime} .
$$

Let $\left(\kappa_{0},\left[u_{1}, u_{2}\right]\right)=$ fresh $\kappa\left[\bar{T}_{1}, \bar{T}_{2}\right]$ and $\sigma_{0}=\sigma \oplus u_{1} \mapsto v_{p_{1}}$.

As usual, we can immediately use the inductive hypothesis for the predicate derivation of $e$ to obtain $\kappa_{1}, \sigma_{1}, q_{1}$ and $t_{1}$ such that

$$
\begin{array}{r}
\left.\sigma_{1}\right|_{\sigma_{0}} \equiv \sigma_{0}, \quad \sigma_{1} \in \llbracket \kappa_{1} \rrbracket \text { and } \\
L_{\bar{T}_{1}+\bar{T}_{2}} u_{1} \Leftarrow e_{1}=\kappa_{0} \Uparrow_{q_{1}}^{t_{1}}\left\{\kappa_{1}\right\} .
\end{array}
$$

However, we can't conclude that there exists a similar derivation for $R_{\bar{T}_{1}+\bar{T}_{2}} u_{2}$ from some inductive hypothesis since we don't have a corresponding derivation! That's where the termination assumptions comes in: by the Termination Lemma (Lemma 3.6.26) there exists some $\kappa^{?}$ such that $R_{\bar{T}_{1}+\bar{T}_{2}} u_{2} \Leftarrow e_{1}=\kappa_{0} \Uparrow_{q_{2}}^{t_{2}} \kappa$.

We now do case analysis on $\kappa^{\text {? }}$. If it is equal to $\varnothing$, then the proof is straightforward following rule M-Case-3, using the inductive hypothesis for the other predicate derivation.

If, on the other hand, $\kappa^{?}=\left\{\kappa_{2}\right\}$ for some $\kappa_{2}$, we face a similar problem for the second derivation. We can obtain $\kappa_{a}, \sigma_{a}, q_{1}^{\prime}$ and $t_{1}^{\prime}$ such that

$$
\begin{gathered}
\left.\sigma_{a}\right|_{\sigma_{1}} \equiv \sigma_{1}, \quad \sigma_{a} \in \llbracket \kappa_{a} \rrbracket \text { and } \\
p \Leftarrow e_{1}\left[u_{1} / x\right]=\kappa_{1} \Uparrow_{q_{1}^{\prime}}^{t_{1}^{\prime}}\left\{\kappa_{a}\right\}
\end{gathered}
$$

by the inductive hypothesis for the derivation of $e_{p_{1}}\left[v_{p_{1}} / x\right]$, but we have no corresponding derivation for the other branch. Using the Termination Lemma once again, we can obtain the there exists some such $\kappa_{b}^{?}$.

Once again we do case analysis on $\kappa_{b}^{?}$. If $\kappa_{b}^{?}=\varnothing$ then the branch of combine that fires returns $\left\{\kappa_{a}\right\}$ and the result follows directly. If $\kappa_{b}^{?}=\left\{\kappa_{b}\right\}$ for some $\kappa_{b}$, then, by the specification of union, $\sigma_{a}$ is contained in the denotation of the combination and the result follows.

\section{Implementation}

We next describe the Luck prototype: its top level, its treatment of backtracking, and the implementation of primitive integers instantiating the abstract specification presented in $\$ 3$

At the Top Level The inputs provided to the Luck interpreter consist of an expression $e$ of type bool containing zero or more free unknowns $\vec{u}$ (but no free variables), and an initial constraint set $\kappa$ providing types and finite domains ${ }^{3}$ for each unknown in $\vec{u}$, such that their occurrences in $e$ are well typed $(\varnothing ; U(\kappa) \vdash e: 1+1)$.

The interpreter matches $e$ against True (that is, $\left.L_{1+1}()\right)$, to derive a refined constraint set $\kappa^{\prime}$ :

$$
L_{1+1}() \Leftarrow e=\kappa \Uparrow_{q}^{t}\left\{\kappa^{\prime}\right\}
$$

\footnotetext{
${ }^{3}$ This restriction to finite domains appears to be crucial for our technical development to work, as discussed in the previous section. In practice, we have not yet encountered a situation where it was important to be able to generate examples of unbounded size (as opposed to examples up to some large maximum size). We do sometimes want to generate structures containing large numbers, since they can be represented efficiently, but here, too, choosing an enormous finite bound appears to be adequate for the applications we've tried. The implementation allows for representing all possible ranges of a corresponding type up to a given size bound. Such bounds are initialized at the top level, and they are propagated (and reduced a bit) to fresh unknowns created by pattern matching before these unknowns are used as inputs to the interpreter.
}

This involves random choices, and there is also the possibility that matching fails (and the semantics generates $\varnothing$ instead of $\left\{\kappa^{\prime}\right\}$ ). In this case, a simple global backtracking approach could simply try the whole thing again (up to an ad hoc limit). While not strictly necessary for a correct implementation of the matching semantics, some local backtracking allows wrong choices to be reversed quickly and leads to an enormous improvement in performance [19]. Our prototype backtracks locally in calls to choose: if choose has two choices available and the first one fails when matching the instantiated expression against a pattern, then we immediately try the second choice instead. Effectively, this means that if $e$ is already known to be of the form $L_{-}$, then narrow will not choose to instantiate it using $R_{-}$, and vice versa. This may require matching against $e$ twice, and our implementation shares work between these two matches as far as possible. (It also seems useful to give the user explicit control over where backtracking occurs, but we leave this for future work.)

After the interpreter matches $e$ against True, all the resulting valuations $\sigma \in \llbracket \kappa^{\prime} \rrbracket$ should map the unknowns in $\vec{u}$ to some values. However, there is no guarantee that the generator semantics will yield a $\kappa^{\prime}$ mapping every $\vec{u}$ to a unique values. The Luck top-level then applies the sample constraint set function to each unknown in $\vec{u}$, ensuring that $\left.\sigma\right|_{\vec{u}}$ is the same for each $\sigma$ in the final constraint set. The interpreter returns this common $\left.\sigma\right|_{\vec{u}}$ if it exists, and backtracks otherwise.

Pattern Match Compiler In Section 2, we saw an example using a standard Tree datatype and instantiation expressions assigning different weights to each branch. While the desugaring of simple pattern matching to core Luck syntax is straightforward 3.1 , nested patterns-as in Fig. 16-complicate things in the presence of probabilities. We expand such expressions to a tree of simple case expressions that match only the outermost constructors of their scrutinees. However, there is generally no unique choice of weights in the expanded predicate: a branch from the source predicate may be duplicated in the result. We guarantee the intuitive property that the sum of the probabilities of the clones of a branch is proportional to the weights given by the user, but that still does not determine the individual probabilities that should be assigned to these clones.

The most obvious way to distribute weights is to simply share the weight equally with all duplicated branches. But the probability of a single branch then depends on the total number of expanded branches that come from the same source, which can be hard for users to determine and can vary widely even between sets of patterns that appear similar. Instead, Luck's default weighing strategy works as follows. For any branch $B$ from the source, at any intermediate case expression of the expansion, the subprobability distribution over the immediate subtrees that contain at least one branch derived from $B$ is uniform. This makes modifications of the source patterns in nested positions affect the distribution more locally.

In Figure 16, the False branch should have probability $\frac{1}{3}$. It is expanded into four branches, corresponding to subpatterns Var ${ }_{-}$, Lam _ _, App (Var _) _, App (App _ _ ) . The latter two are grouped under the pattern App _ _., while the former two are in their own groups. These three groups receive equal shares of the total probability of the original branch, that is $\frac{1}{9}$ each. The two branches for App (Var _) _ and App (App _ _) _ split that further into twice $\frac{1}{18}$. On the other hand, True remains a single branch with probability $\frac{2}{3}$. The weights on the left of every pattern are calculated to reflect this distribution.

Constraint Set Implementation Our desugaring of source-level pattern matching to core case expressions whose discriminee $e$ is first narrowed means that rule M-Case-1 is not executed for datatypes; only one of the evaluations of $e$ against the $L$ and $R$ 


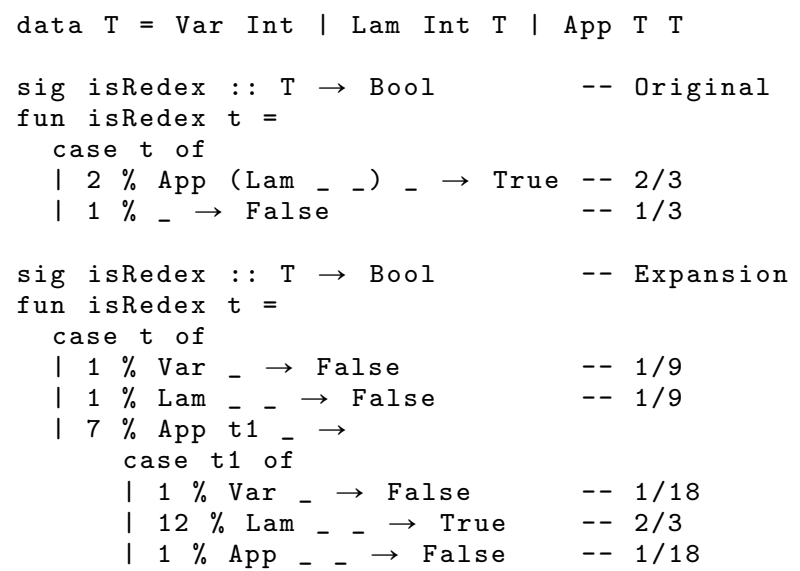

Figure 16. Expanding case expression with a nested pattern and a wildcard. Comments show the probability of each alternative.

patterns will succeed and only one branch will be executed. This means that our constraint-set representation for datatypes doesn't need to implement union. We leverage this to provide a simple and efficient implementation of the unification constraints. For our prototype, the constraint solving behavior of case is only exploited in our treatment of primitive integers, which we detail at the end of this section.

The constraint set interface could be implemented in a variety of different ways. The simplest would be to explicitly represent constraint sets as sets of valuations, but this would lead to efficiency problems, since even unifying two unknowns would require traversing the whole set, filtering out all valuations in which the unknowns are different. On the other extreme, we could represent a constraint set as an arbitrary logical formula over unknowns. While this is a compact representation, it does not directly support the pervariable sampling that we require.

For our prototype we choose a middle way, using a simple data structure we call orthogonal maps to represent sets of valuations. An orthogonal map is a map from unknowns to ranges, which have the following syntax:

$$
r::=()|u|(r, r) \mid \text { fold } r|L r| R r \mid\{L r, R r\}
$$

Ranges represent sets of non-functional values: units, unknowns, pairs of ranges, and $L$ and $R$ applied to ranges. We also include the option for a range to be a pair of an $L$ applied to some range and an $R$ applied to another. For example, the set of all Boolean values can be encoded compactly in a range (eliding folds and type information) as $\{L(), R()\}$. Similarly, the set $\{0,2,3\}$ can be encoded as $\{L(), R(R\{L(), R()\})\}$, assuming a standard Peano encoding of natural numbers.

However, while this compact representation can represent all sets of naturals, not all sets of Luck non-functional values can be precisely represented. For instance the set $\{(0,1),(1,0)\}$ cannot be represented using ranges, only approximated to $(\{L(), R(L())\}$, $\{L(), R(L())\})$, which represents the larger set $\{(0,0),(0,1),(1$, $0),(1,1)\}$. This corresponds to a form of Cartesian abstraction, in which we lose any relation between the components of a pair, so if one used ranges as an abstract domain for abstract interpretation it would be hard to prove say sortedness of lists. Ranges are a rather imprecise abstract domain for algebraic datatypes [43, 45, 53].

We implement constraint sets as pairs of a typing environment and an optional map from unknowns to ranges. The typing environment of a constraint set $(U(\cdot)$ operation), is just the first projection of the tuple. A constraint set $\kappa$ is $S A T$ if the second element is not $\varnothing$. The sample primitive indexes into the map and collects all possible values for an unknown.

The only interesting operation with this representation is unify. It is implemented by straightforwardly translating the values to ranges and unifying those. For simplicity, unification of two ranges $r_{1}$ and $r_{2}$ in the presence of a constraint set $\kappa$ returns both a constraint set $\kappa^{\prime}$ where $r_{1}$ and $r_{2}$ are unified and the unified range $r^{\prime}$. If $r_{1}=r_{2}=()$ there is nothing to be done. If both ranges have the same top-level constructor, we recursively unify the inner subranges. If one of the ranges, say $r_{1}$, is an unknown $u$ we index into $\kappa$ to find the range $r_{u}$ corresponding to $u$, unify $r_{u}$ with $r_{2}$ in $\kappa$ to obtain a range $r^{\prime}$, and then map $u$ to $r^{\prime}$ in the resulting constraint set $\kappa^{\prime}$. If both ranges are unknowns $u_{1}, u_{2}$ we unify their corresponding ranges to obtain $r^{\prime}$. We then pick one of the two unknowns, say $u_{1}$, to map to $r^{\prime}$, while mapping $u_{2}$ to $u_{1}$. To keep things deterministic we introduce an ordering on unknowns and always map $u_{i}$ to $u_{j}$ if $u_{i}<u_{j}$. Finally, if one range is the compound range $\left\{L r_{1 l}, R r_{1 r}\right\}$ while the other is $L r_{2}$, the resulting range is only $L$ applied to the result of the unification of $r_{1 l}$ and $r_{2}$.

It is easy to see that if we start with a set of valuations that is representable as an orthogonal map, non-union operations will result in constraint sets whose denotation is still representable, which allows us to get away with this simple implementation of datatypes. The M-Case-1 rule is used to model our treatement of integers. We introduce primitive integers in our prototype accompanied by standard integer equality and inequality constraints. In Section 3.5 we saw how a recursive less-than function can be encoded using Peano-style integers and case expressions that do not contain instantiation expressions in the discriminee. All integer constraints can be desugared into such recursive functions with the exact same behavior-modulo efficiency.

To implement integer constraints, we extend the codomain of the mapping in the constraint set implementation described above to include a compact representation of sets of intervals of primitive integers as well as a set of the unknown's associated constraints. Every time the domain of an unknown $u$ is refined, we use an incremental variant of the AC-3 arc consistency algorithm [49] to efficiently refine the domains of all the unknowns linked to $u$, first iterating through the constrains associated with $u$ and then only through the constraints of other "affected" unknowns.

\section{Evaluation}

To evaluate the expressiveness and efficiency of Luck's hybrid approach to test case generation, we tested it with a number of small examples and two significant case studies: generating welltyped lambda terms and information-flow-control machine states. The Luck code is generally much smaller and cleaner than that of existing handwritten generators, though the Luck interpreter takes longer to generate each example - around $20 \times$ to $24 \times$ for the more complex generators. (Also, while this is admittedly a subjective impression, we found it significantly easier to get the generators right in Luck.)

Small Examples The literature on random test generation includes many small examples-list predicates such as sorted, member, and distinct, tree predicates like BSTs $\$ 2$ and redblack trees, and so on. In Appendix A.1 we show the implementation of many such examples in Luck, illustrating how we can write predicates and generators together with minimal effort.

We use red-black trees to compare the efficiency of our Luck interpreter to generators provided by commonly used tools like QuickCheck (random testing), SmallCheck (exhaustive testing) and Lazy SmallCheck [65]. Lazy SmallCheck leverages Haskell's laziness to greatly improve upon out-of-the-box QuickCheck and 


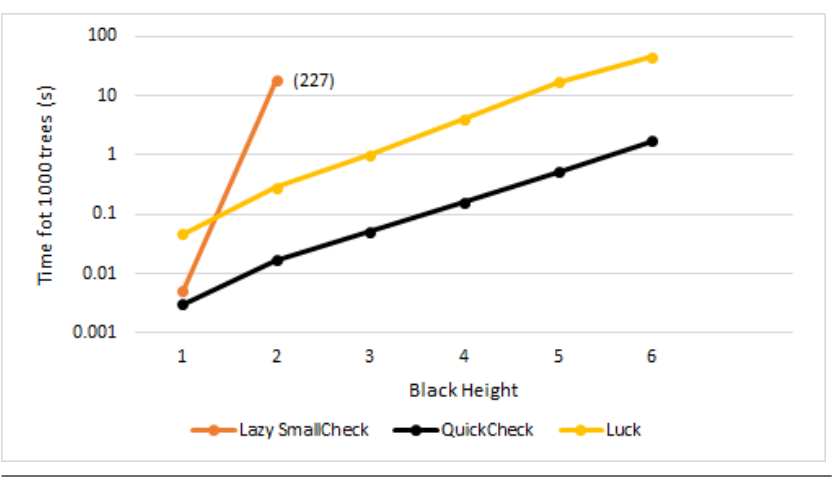

Figure 17. Red-Black Tree Experiment

SmallCheck generators in the presence of sparse preconditions, by using partially defined inputs to explore large parts of the search space at once. Using both Luck and Lazy SmallCheck, we attempted to generate 1000 red black trees with a specific black height $b h$-meaning that the depth of the tree can be as large as $2 \cdot b h+1$. Results are shown in Fig. 17 Lazy SmallCheck was able to generate all 227 trees of black height 2 in 17 seconds, fully exploring all trees up to depth 5 . When generating trees of black height 3, which required exploring trees up to depth 7, Lazy SmallCheck was unable to generate 1000 red black trees within 5 minutes. At the same time, the Luck implementation lies consistently within an order of magnitude of a very efficient handwritten QuickCheck generator that generates valid Red-Black trees directly. Using rejection-sampling approaches by generating trees and discarding those that don't satisfy the red-black tree invariant (e.g., QuickCheck or SmallCheck's ==>) is prohibitively costly: these approaches perform much worse than Lazy SmallCheck.

Well-Typed Lambda Terms Using our prototype implementation we reproduced the experiments of Pałka et al. [60], who generated well-typed lambda terms in order to discover bugs in GHC's strictness analyzer. We also use this case study to indirectly compare to two narrowing-based tools that are arguably closer to Luck and that use the same case study to evaluate their work: Claessen et al. [18, 19] and Fetscher et al. [25].

We encoded a model of simply typed lambda calculus with polymorphism in Luck, providing a large typing environment with standard functions from the Haskell Prelude to generate interesting well-typed terms. The generated ASTs were then pretty-printed into Haskell syntax and each one was applied to a partial list of the form: [1,2, undefined]. Using the same version of GHC (6.12.1), we compiled each application twice: once with optimizations (-02) and once without and compared the outputs.

A straightforward Luck implementation of a type system for the polymorphic lambda calculus was not adequate for finding bugs efficiently. To improve its performance we borrowed tricks from the similar case study of Fetscher et al. [25], seeding the environment with monomorphic versions of possible constants and increasing the frequency of seq, a basic Haskell function that introduces strictness, to increase the chances of exercising the strictness analyzer. Using this, we discovered bugs that seem similar (under quick manual inspection) to those found by Pałka et al. and Fetscher et al..

Luck's generation speed was slower than that of Pałka's handwritten generator. We were able to generate terms of average size 50 (internal nodes), and, grouping terms together in batches of 100 , we got a total time of generation, unparsing, compilation and execution of around 35 seconds per batch. This is a slowdown of 20x compared to that of Pałka's. However, our implementation is a total of 82 lines of fairly simple code, while the handwritten devel- opment is 1684 lines, with the warning "...the code is difficult to understand, so reading it is not recommended" in its distribution page [59].

The derived generators of Claessen et al. [18] achieved a 7x slowdown compared to the handwritten generator, while the Redex generators [25] also report a $7 x$ slowdown in generation time for their best generator. However, by seeding the environment with monomorphised versions of the most common constants present in the counterexamples, they were able to achieve a time per counterexample on par with the handwritten generator.

Information-Flow Control For a second large case study, we re-implemented a method for generating information-flow-control machine states [39]. Given an abstract stack machine with data and instruction memories, a stack, and a program counter, one attaches labels - security levels - to runtime values, propagating them during execution and restricting potential flows of information from high (secret) to low (public) data. The desired security property, termination-insensitive noninterference, states that if we start with two indistinguishable abstract machines s1 and s2 (i.e., all their low-tagged parts are identical) and run each of them to completion, then the resulting states s1' and s2' are also indistinguishable.

Hriţcu et al. [39] found that efficient testing of this property could be achieved in two ways: either by generating instruction memories that allow for long executions and checking for indistinguishability at each low step (called $L L N I$, low-lockstep noninterference), or by looking for counter-examples to a stronger invariant (strong enough to prove noninterference), generating two arbitrary indistinguishable states and then running for a single step (SSNI, single step noninterference). In both cases, there is some effort involved in generating indistinguishable machines: for efficiency, one must first generate one abstract machine $\mathrm{s}$ and then vary $\mathrm{s}$, to generate an indistinguishable one s'. In writing such a generator for variations, one must effectively reverse the indistinguishability predicate between states and then keep the two artifacts in sync.

We first investigated the stronger property (SSNI), by encoding the indistinguishability predicate in Luck and using our prototype to generate small, indistinguishable pairs of states. In 216 lines of code we were able to describe both the predicate and the generator for indistinguishable machines. The same functionality required $>1000$ lines of complex Haskell code in the handwritten version. The handwritten generator is reported to generate an average of 18400 tests per second, while the Luck prototype generates an average of 1450 tests per second, around 12.5 times slower.

The real promise of Luck, however, became apparent when we turned to LLNI. Hriţcu et al. [39] generate long sequences of instructions using generation by execution: starting from a machine state where data memories and stacks are instantiated, they generate the current instruction ensuring it does not cause the machine to crash, then allow the machine to take a step and repeat. While intuitively simple, this extra piece of generator functionality took significant effort to code, debug, and optimize for effectiveness, resulting in more than 100 additional lines of code. The same effect was achieved in Luck by the following 6 intuitive lines, where we just put the previous explanation in code:

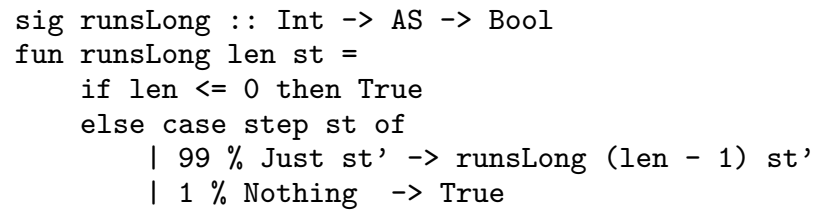

We evaluated our generator on the same set of buggy informationflow analyses as in Hriţcu et al. [39]. We were able to find all of the same bugs, with similar effectiveness (number of bugs found per 100 tests). However, the Luck generator was 24 times slower 
(Luck: 150 tests/s, Haskell: 3600 tests/s). We expect to be able to improve this result (and the rest of the results in this section) with a more efficient implementation that compiles Luck programs to QuickCheck generators directly, instead of interpreting them in a minimally tuned prototype.

The success of the prototype in giving the user enough flexibility to achieve similar effectiveness with state-of-the-art generators, while significantly reducing the amount of code and effort required, suggests that the approach Luck takes is promising and points towards the need for a real, optimizing implementation.

\section{Related Work}

Luck lies in the intersection of many different topics in programming languages, and the potentially related literature is huge. Here, we present just the closest related work. Afterwards, we demonstrate how a Luck user can leverage Boltzmann samplers to achieve uniformity guarantees.

Random Testing The works that are most closely related to our own are the narrowing based approaches of Gligoric et al. [29], Claessen et al. [18, 19] and Fetscher et al. [25]. Gligoric et al. use a "delayed choice" approach, which amounts to needed-narrowing, to generate test cases in Java. Claessen et al. exploit the laziness of Haskell, combining a narrowing-like technique with FEAT [23], a tool for functional enumeration of algebraic types, to efficiently generate near-uniform random inputs satisfying some precondition. While their use of FEAT allows them to get uniformity by default, it is not clear how user control over the resulting distribution could be achieved. Fetscher et al. [25] also use an algorithm that makes local choices with the potential to backtrack in case of failure. Moreover, they add a simple version of constraint solving, handling equality and disequality constraints. This allows them to achieve excellent performance in testing GHC for bugs (as in [60|) using the "trick" of monomorphizing the polymorphic constants of the context as discussed in the previous section. They present two different strategies for making local choices: uniformly at random, or by ordering branches based on their branching factor. While both of these strategies seem reasonable (and somewhat complementary), there is no way of exerting control over the distribution as necessary.

Enumeration-Based Testing An interesting related approach appears in the inspiring work of Bulwahn [7]. In the context of Isabelle's [54] QuickCheck [6], Bulwahn automatically constructs enumerators for a given precondition via a compilation to logic programs using mode inference. This work successfully addresses the issue of generating satisfying valuations for preconditions directly and serves for exhaustive testing of "small" instances, significantly pushing the limit of what is considered "small" compared to previous approaches. Lindblad [48] and Runciman et al. [65] also provide support for exhaustive testing using narrowing-based techniques. Instead of implementing mechanisms that resemble narrowing in standard functional languages, Fischer and Kuchen [26] leverage the built-in engine of the functional logic programming language Curry [36] to enumerate tests satisfying a coverage criterion. In a later, black-box approach for Curry, Christiansen and Fischer [17] additionally use level diagonalization and randomization to bring larger tests earlier in the enumeration order. While exhaustive testing is useful and has its own merits and advantages over random testing in a lot of domains, we turn to random testing because the complexity of our applications - testing noninterference or optimizing compilers-makes enumeration impractical.

Constraint Solving Many researchers have turned to constraintsolving based approaches to generate random inputs satisfying preconditions. In the constraint solving literature around SAT witness generation, the pioneering work of Chakraborty et al. [15] stands out because of its efficiency and its guarantees of approximate uniformity. However, there is no way-and no obvious way to add it —of controlling distributions. In addition, their efficiency relies crucially on the independent support being small relative to the entire space (where the support $X$ of a boolean formula $\mathrm{p}$ is the set of variables appearing in $\mathrm{p}$ and the independent support is a subset $D$ of $X$ such that no two satisfying assignments for $\mathrm{p}$ differ only in $X \backslash D$ ). While true for typical SAT instances, this is not the case for random testing properties, like, for example, noninterference. In fact, a minimal independent support for indistinguishable machines includes one entire machine state and the high parts of another; thus, the benefit from their heuristics may be minimal. Finally, they require logical formulae as inputs, which would require a rather heavy translation from a high-level language like Haskell.

Such a translation from a higher-level language to the logic of a constraint solver has been attempted a few times to support testing [13, 34], the most recent and efficient for Haskell being Target [66]. Target translates preconditions in the form of refinement types, and uses a constraint solver to generate a satisfying valuation for testing. Then it introduces the negation of the generated input to the formula, in order to generate new, different ones. While more efficient than Lazy SmallCheck in a variety of cases, there are still cases where a narrowing-like approach outperforms the tool, further pointing towards the need to combine the two approaches as in Luck. Moreover, the use of an automatic translation and constraint solving does not give any guarantees on the resulting distribution, neither does it allow for user control.

Constraint-solving is also used in symbolic evaluation based techniques, where the goal is to generate diverse inputs that achieve higher coverage [3, 8, 11, 30, 31, 50, 67]. Recently, in the context of Rosette [70], symbolic execution was used to successfully find bugs in the same information-flow control case study.

Semantics for narrowing-based solvers Recently, Fowler and Hutton [27] put needed-narrowing based solvers on a firmer mathematical foundation. They presented an operational semantics of a purely narrowing-based solver, named Reach, proving soundness and completeness. In their concluding remarks, they mention that native representations of primitive datatypes do not fit with the notion of lazy narrowing since they are "large, flat datatypes with strict semantics." In Luck, we were able to exhibit the same behavior for both the primitive integers and their datatype encodings successfully addressing this issue, while at the same time incorporating constraint solving into our formalization.

Probabilistic programming Semantics for probabilistic programs share many similarities with the semantics of Luck [32, 33 52], while the problem of generating satisfying valuations shares similarities with probabilistic sampling [14, 47, 51, 55]. For example, the semantics of the languagePROB in the recent probabilistic programming survey of Gordon et al. [33] takes the form of probability distributions over valuations, while Luck semantics can be viewed as (sub)probability distributions over constraint sets, which induces a distribution over valuations. Moreover, in probabilistic programs, observations serve a similar role to preconditions in random testing, creating problems for simplistic probabilistic samplers that use rejection sampling-i.e., generate and test. Recent advances in this domain, like the work on Microsoft's R2 Markov Chain Monte Carlo sampler [55], have shown promise in providing more efficient sampling, using pre-imaging transformations in analyzing programs. An important difference is in the type of programs usually targeted by such tools. The difficulty in probabilistic programming arises mostly from dealing with a large number of complex observations, modeled by relatively small programs. For example, Microsoft's TrueSkill [38] ranking program is a very small program, powered by millions of observations. In contrast, 


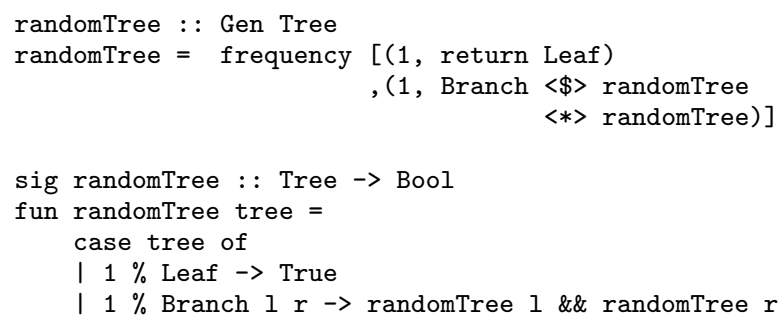

Figure 18. Simple tree generator (Haskell vs Luck)

random testing deals with very complex programs (e.g., a type checker) and a single observation without noise (observe true).

We did a simple experiment with R2, using the following probabilistic program to model the indistinguishability of $\$ 2$ where we use booleans to model labels:

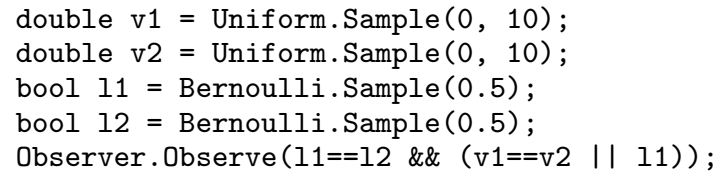

Two pairs of doubles and booleans will be indistinguishable if the booleans are equal and, if the booleans are false, so are the doubles. The result was somewhat surprising at first, since all the generated samples have their booleans set to true. However, that is an accurate estimation of the posterior distribution: for every "false" indistinguishable pair there exist $2^{64}$ "true" ones! Of course, one could probably come up with a better prior or use a tool that allows arbitrary conditioning to skew the distribution appropriately. If, however, for such a trivial example the choices are non-obvious, imagine replacing pairs of doubles and booleans with arbitrary lambda terms and indistinguishability by a well-typedness relation. Coming up with suitable priors that lead to efficient testing would become an ambitious research problem on its own!

Boltzmann Samplers Boltzmann samplers for algebraic datatypes work by making appropriately weighted local choices based on a control parameter. In the binary tree case, a Boltzmann sampler would look similar to a simplistic generator for trees: flip a (potentially biased) coin, generating a Leaf or a Node based on the outcome; then recurse (Fig. 18). The distribution this generator induces can be easily implemented by a trivial Luck predicate, also in Fig. 18 A Boltzmann sampler is a slightly modified version of this simple approach. First of all, the bias in the local choices must be systematically calculated (or approximated with numerical methods). This bias depends on the convergence of a generating function associated with the data type's recursive specification. Then, we start sampling using the computed bias for each choice. If at any point we reach a size bigger than $n(1+\epsilon)$, we stop the generation and try again. If the generation stops with a term $n(1-\epsilon)$, we throw away the generated term and try again. The theory behind Boltzmann samplers guarantees that this approach will terminate in expected linear time in $n$ (including discards!), and the result will be uniformly selected among other elements of the same size.

Using Boltzmann samplers is a natural fit for our setting and requires two things: a way to compute the control parameter for the structure that is being generated, and a way to reject samples that are outside the neighborhood of the desired size. Both of these can be handled automatically, as shown by Canou and Darrasse [12].

The presence of additional constraints on generated data could skew the posterior distributions, negating any uniformity guarantees. But if, every time we reach an unsatisfiable constraint, we backtrack to the beginning of the generation, uniformity is pre- served since we are only concerned with uniformity in the set of satisfying valuations. Unfortunately, throwing away all progress is not efficient; it is usually a lot faster to backtrack to a more recent choice instead of the beginning. Such an algorithm still gives us some assurance about the distribution, similarly to Claessen et al. [18]: the least likely value generated will be at most a constant factor less likely than the most likely one, where the factor is the amount of local backtracking allowed.

\section{Conclusions and Future Work}

We have presented Luck, a language for writing generators in the form of lightly annotated predicates. We presented the semantics of Luck, combining local instantiation and constraint solving in a unified framework and exploring their interactions. We described a prototype implementation of this semantics, which we used to replicate the results of state-of-the-art handwritten random generators for two complex domains. The results showed the potential of Luck's approach, allowing us to replicate the generation presented by the handwritten generators with reduced code and effort. The prototype was slower by an order of magnitude, but there is still significant room for improvement.

In the future it will be interesting to explore compilation of Luck into generators in a language like Haskell to improve the performance of our interpreted prototype. Another way to improve performance would be to experiment with other domain representations. We also want to investigate Luck's equational theory, showing, for instance, that the encoded conjunction, negation, and disjunction satisfy the usual logical laws. Finally, the backtracking strategies in our implementation can be abstractly modeled on top of our notion of choice-recording trace; Gallois-Wong [28] shows promising preliminary results using Markov chains for this.

Another potential direction for future work is automatically deriving smart shrinkers. Shrinking, or delta-debugging, is crucial in property-based testing, and it can also require significant user effort and domain specific knowledge to be efficient [62]. It would be interesting to see if there is a counterpart to narrowing or constraint solving that allows shrinking to preserve desired properties.

Finally, we would like to see if transferring ideas from Luck to generate inductive datatypes for testing in the Coq proof assistant [21, 61] can ease the formalization effort, allowing users to discover flaws early in the proof process as in other proof assistants [6, 16].

\section{Acknowledgments}

We are grateful to Maxime Dénès, Nate Foster, Thomas Jensen, Gowtham Kaki, George Karachalias, Michał Pałka, Zoe Paraskevopoulou, Christine Rizkallah, Antal Spector-Zabusky, Perdita Stevens, and Andrew Tolmach for useful comments. This work was supported by NSF awards \#1421243, Random Testing for Language Design and \#1521523, Expeditions in Computing: The Science of Deep Specification. This work was, in part, supported by the European Research Council under ERC Starting Grant SECOMP (715753). 


\section{A. Appendix}

\section{A.1 Luck examples}

In this appendix we present the Luck programs that serve as both predicates and generators for the small examples of $\$ 5$

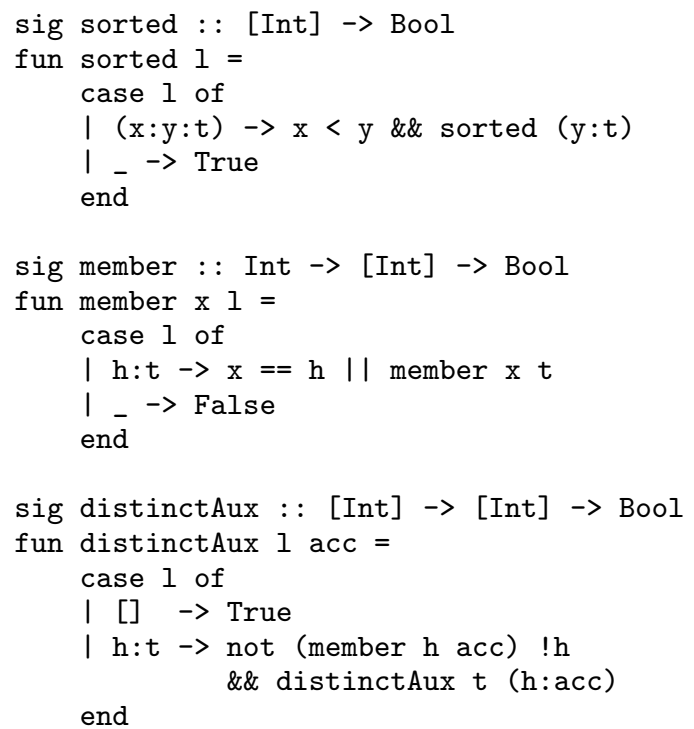

sig distinct : : [Int] $\rightarrow$ Bool

fun distinct $1=\operatorname{aux} 1$ []

In order to obtain lists of a specific size, we could skew the distribution towards the cons case using numeric annotations on the branches, or, we can use the conjunction of such a predicate with the following simple length predicate (which could be greatly simplified with some syntactic sugar).

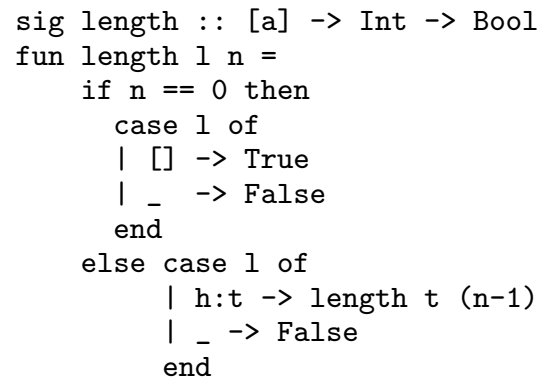

Finally, the Luck program that generates red black trees of a specific height is:

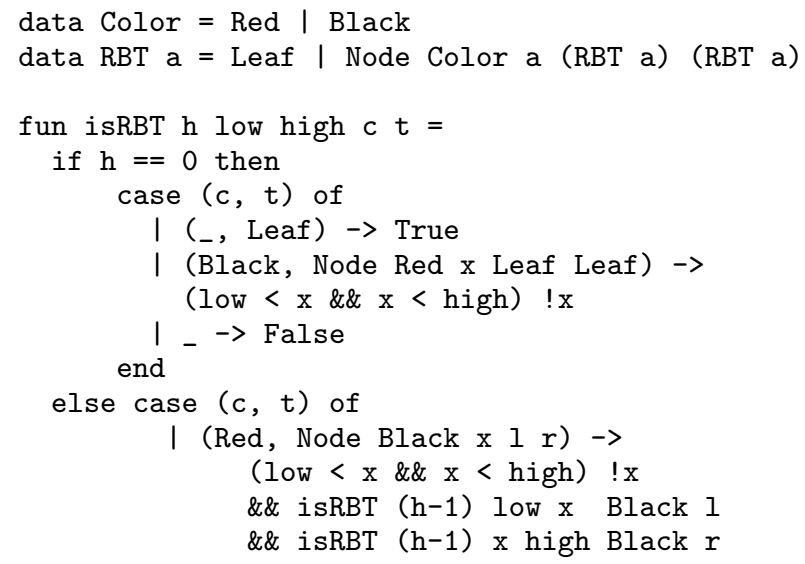

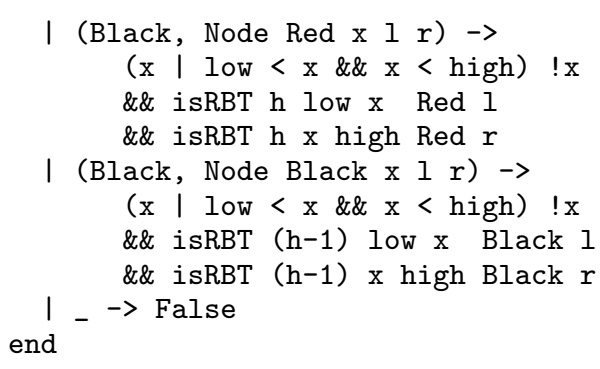

\section{References}

[1] S. Antoy. A needed narrowing strategy In Journal of the ACM. 2000.

[2] T. Arts, L. M. Castro, and J. Hughes. Testing Erlang data types with QuviQ QuickCheck In 7th ACM SIGPLAN Workshop on Erlang. 2008.

[3] T. Avgerinos, A. Rebert, S. K. Cha, and D. Brumley. Enhancing symbolic execution with Veritesting In 36th International Conference on Software Engineering, ICSE '14, Hyderabad, India - May 31 - June 07, 2014, 2014.

[4] T. Ball, V. Levin, and S. K. Rajamani. A decade of software model checking with SLAM Commun. ACM, 54(7):68-76, 2011.

[5] J. C. Blanchette and T. Nipkow. Nitpick: A counterexample generator for higher-order logic based on a relational model finder In First International Conference on Interactive Theorem Proving (ITP). 2010.

[6] L. Bulwahn. The new Quickcheck for Isabelle - random, exhaustive and symbolic testing under one roof In 2nd International Conference on Certified Programs and Proofs (CPP). 2012.

[7] L. Bulwahn. Smart testing of functional programs in Isabelle In 18th International Conference on Logic for Programming, Artificial Intelligence, and Reasoning (LPAR). 2012.

[8] J. Burnim and K. Sen. Heuristics for scalable dynamic test generation In 23rd IEEE/ACM International Conference on Automated Software Engineering (ASE), 2008.

[9] C. Cadar, D. Dunbar, and D. Engler. KLEE: unassisted and automatic generation of high-coverage tests for complex systems programs In 8 th USENIX conference on Operating systems design and implementation. 2008.

[10] C. Cadar, V. Ganesh, P. M. Pawlowski, D. L. Dill, and D. R. Engler EXE: automatically generating inputs of death In 13 th ACM conference on Computer and communications security. 2006.

[11] C. Cadar, P. Godefroid, S. Khurshid, C. S. Păsăreanu, K. Sen, N. Tillmann, and W. Visser. Symbolic execution for software testing in practice: preliminary assessment In 33rd International Conference on Software Engineering. 2011.

[12] B. Canou and A. Darrasse. Fast and sound random generation for automated testing and benchmarking in objective caml Workshop on ML, 2009.

[13] M. Carlier, C. Dubois, and A. Gotlieb. Constraint reasoning in FocalTest In 5th International Conference on Software and Data Technologies. 2010.

[14] A. T. Chaganty, A. V. Nori, and S. K. Rajamani. Efficiently sampling probabilistic programs via program analysis In Artificial Intelligence and Statistics (AISTATS), 2013

[15] S. Chakraborty, K. S. Meel, and M. Y. Vardi. Balancing scalability and uniformity in SAT witness generator In Proceedings of the 51st Annual Design Automation Conference. 2014

[16] H. R. Chamarthi, P. C. Dillinger, M. Kaufmann, and P. Manolios. Integrating testing and interactive theorem proving In 10th International Workshop on the ACL2 Theorem Prover and its Applications, 2011

[17] J. Christiansen and S. Fischer. EasyCheck - test data for free In 9th International Symposium on Functional and Logic Programming (FLOPS). 2008

[18] K. Claessen, J. Duregård, and M. H. Pałka. Generating constrained random data with uniform distribution In Functional and Logic Programming. 2014. 
[19] K. Claessen, J. Duregård, and M. H. Palka. Generating constrained random data with uniform distribution J. Funct. Program., 25, 2015.

[20] K. Claessen and J. Hughes. QuickCheck: a lightweight tool for random testing of Haskell programs In 5th ACM SIGPLAN International Conference on Functional Programming (ICFP). 2000.

[21] M. Dénès, C. Hriţcu, L. Lampropoulos, Z. Paraskevopoulou, and B. C. Pierce. QuickChick: Property-based testing for Coq The Coq Workshop, 2014.

[22] P. Duchon, P. Flajolet, G. Louchard, and G. Schaeffer. Boltzmann samplers for the random generation of combinatorial structures $\mathrm{Com}$ binatorics, Probability \& Computing, 13(4-5):577-625, 2004.

[23] J. Duregård, P. Jansson, and M. Wang. Feat: Functional enumeration of algebraic types In Proceedings of the 2012 Haskell Symposium. 2012.

[24] P. Dybjer, Q. Haiyan, and M. Takeyama. Combining testing and proving in dependent type theory In 16th International Conference on Theorem Proving in Higher Order Logics (TPHOLs). 2003.

[25] B. Fetscher, K. Claessen, M. H. Palka, J. Hughes, and R. B. Findler. Making random judgments: Automatically generating well-typed terms \begin{tabular}{|l|l|}
\hline from the definition of a type-system & In 24th European Symposium on
\end{tabular} Programming. 2015.

[26] S. Fischer and H. Kuchen. Systematic generation of glass-box test cases for functional logic programs In 9th International ACM SIGPLAN Conference on Principles and Practice of Declarative Programming (PPDP). 2007.

[27] J. Fowler and G. Huttom. Towards a theory of reach In Trends in Functional Programming - 16th International Symposium, TFP 2015, Sophia Antipolis, France, June 3-5, 2015. Revised Selected Papers. 2015.

[28] D. Gallois-Wong. Formalising Luck: Improved probabilistic semantics for property-based generators Inria Internship Report, 2016.

[29] M. Gligoric, T. Gvero, V. Jagannath, S. Khurshid, V. Kuncak, and D. Marinov. Test generation through programming in UDITA In 32nd ACM/IEEE International Conference on Software Engineering. 2010.

[30] P. Godefroid, N. Klarlund, and K. Sen. DART: directed automated random testing In ACM SIGPLAN Conference on Programming Language Design and Implementation. 2005.

[31] P. Godefroid, M. Y. Levin, and D. A. Molnar. SAGE: whitebox fuzzing for security testing ACM Quеие, 10(1):20, 2012.

[32] N. D. Goodman, V. K. Mansinghka, D. M. Roy, K. Bonawitz, and J. B. Tenenbaum. Church: a language for generative models In UAI 2008, Proceedings of the 24th Conference in Uncertainty in Artificial Intelligence, Helsinki, Finland, July 9-12, 2008, 2008.

[33] A. D. Gordon, T. A. Henzinger, A. V. Nori, and S. K. Rajamani. Probabilistic programming In J. D. Herbsleb and M. B. Dwyer, editors, Proceedings of the on Future of Software Engineering, FOSE 2014, Hyderabad, India, May 31 - June 7, 2014. 2014.

[34] A. Gotlieb. Euclide: A constraint-based testing framework for critical C programs In ICST 2009, Second International Conference on Software Testing Verification and Validation, 1-4 April 2009, Denver, Colorado, USA, 2009.

[35] A. Groce, C. Zhang, E. Eide, Y. Chen, and J. Regehr. Swarm testing In Proceedings of the 2012 International Symposium on Software Testing and Analysis. 2012.

[36] M. Hanus, H. Kuchen, and J. Moreno-Navarro. Curry: A truly functional logic language In Proc. ILPS'95 Workshop on Visions for the Future of Logic Programming, 1995.

[37] M. Hanus. A unified computation model for functional and logic programming In 24th ACM SIGPLAN-SIGACT Symposium on Principles of Programming Languages (POPL). 1997.

[38] R. Herbrich, T. Minka, and T. Graepel. Trueskill ${ }^{\text {tm }}$ : A bayesian skill rating system In Advances in Neural Information Processing Systems 19, Proceedings of the Twentieth Annual Conference on Neural Information Processing Systems, Vancouver, British Columbia, Canada, December 4-7, 2006, 2006.
[39] C. Hriţcu, J. Hughes, B. C. Pierce, A. Spector-Zabusky, D. Vytiniotis, A. Azevedo de Amorim, and L. Lampropoulos. Testing noninterference, quickly In 18th ACM SIGPLAN International Conference on Functional Programming (ICFP). 2013.

[40] C. Hriţcu, L. Lampropoulos, A. Spector-Zabusky, A. Azevedo de Amorim, M. Dénès, J. Hughes, B. C. Pierce, and D. Vytiniotis. Testing noninterference, quickly Journal of Functional Programming (JFP); Special issue for ICFP 2013, 26:e4 (62 pages), 2016. Technical Report available as arXiv: 1409.0393

[41] J. Hughes. QuickCheck testing for fun and profit In 9th International Symposium on Practical Aspects of Declarative Languages (PADL). 2007.

[42] D. Jackson. Software Abstractions: Logic, Language, and Anlysis The MIT Press, 2011.

[43] T. P. Jensen. Disjunctive program analysis for algebraic data types ACM Trans. Program. Lang. Syst., 19(5):751-803, 1997.

[44] R. Jhala and R. Majumdar. Software model checking ACM Comput. Surv., 41(4), 2009.

[45] G. Kaki and S. Jagannathan. A relational framework for higherorder shape analysis In J. Jeuring and M. M. T. Chakravarty, editors, Proceedings of the 19th ACM SIGPLAN international conference on Functional programming, Gothenburg, Sweden, September 1-3, 2014. 2014.

[46] A. S. Köksal, V. Kuncak, and P. Suter. Scala to the power of Z3: integrating SMT and programming In 23rd International Conference on Automated Deduction. 2011

[47] K. Łatuszyński, G. O. Roberts, and J. S. Rosenthal. Adaptive gibbs samplers and related mcmc methods The Annals of Applied Probability, 23(1):66-98, 2013.

[48] F. Lindblad. Property directed generation of first-order test data In 8th Symposium on Trends in Functional Programming. 2007.

[49] A. K. Mackworth. Consistency in networks of relations Artif. Intell., 8(1):99-118, 1977.

[50] R. Majumdar and K. Sen. Hybrid concolic testing In 29th international conference on Software Engineering. 2007.

[51] V. K. Mansinghka, D. M. Roy, E. Jonas, and J. B. Tenenbaum. Exact and approximate sampling by systematic stochastic search In Proceedings of the Twelfth International Conference on Artificial Intelligence and Statistics, AISTATS 2009, Clearwater Beach, Florida, USA, April 16-18, 2009, 2009.

[52] B. Milch, B. Marthi, S. J. Russell, D. Sontag, D. L. Ong, and A. Kolobov. BLOG: probabilistic models with unknown objects In IJCAI-05, Proceedings of the Nineteenth International Joint Conference on Artificial Intelligence, Edinburgh, Scotland, UK, July 30August 5, 2005, 2005.

[53] F. Nielson and H. R. Nielson. Tensor products generalize the relational data flow analysis method. In 4th Hungarian Computer Science Conference, 1985.

[54] T. Nipkow, M. Wenzel, and L. C. Paulson. Isabelle/HOL: A Proof Assistant for Higher-order Logic. Springer-Verlag, Berlin, Heidelberg, 2002.

[55] A. V. Nori, C.-K. Hur, S. K. Rajamani, and S. Samuel. R2: An efficient mcmc sampler for probabilistic programs In AAAI Conference on Artificial Intelligence (AAAI). 2014.

[56] C. Okasaki. Red-black trees in a functional setting Journal of Functional Programming, 9(4):471-477, 1999.

[57] S. Owre. Random testing in PVS In Workshop on Automated Formal Methods, 2006.

[58] C. Pacheco and M. D. Ernst. Randoop: feedback-directed random testing for Java In 22nd ACM SIGPLAN Conference on Object-Oriented Programming Systems And Applications. 2007.

[59] M. H. Pałka. Testing an optimising compiler by generating random lambda terms. http://www.cse.chalmers.se/ palka/ testingcompiler/ 
[60] M. H. Pałka, K. Claessen, A. Russo, and J. Hughes. Testing an optimising compiler by generating random lambda terms In Proceedings of the 6th International Workshop on Automation of Software Test. 2011.

[61] Z. Paraskevopoulou, C. Hriţcu, M. Dénès, L. Lampropoulos, and B. C. Pierce. Foundational property-based testing In C. Urban and X. Zhang, editors, 6th International Conference on Interactive Theorem Proving (ITP). 2015.

[62] J. Regehr, Y. Chen, P. Cuoq, E. Eide, C. Ellison, and X. Yang. Testcase reduction for $\mathrm{C}$ compiler bugs In ACM SIGPLAN Conference on Programming Language Design and Implementation, PLDI '12, Beijing, China - June 11 - 16, 2012, 2012.

[63] J. S. Reich, M. Naylor, and C. Runciman. Lazy generation of canonical test programs In 23 rd International Symposium on Implementation and Application of Functional Languages. 2011.

[64] A. Rodriguez Yakushev and J. Jeuring. Enumerating well-typed terms generically In U. Schmid, E. Kitzelmann, and R. Plasmeijer, editors, Approaches and Applications of Inductive Programming, volume 5812 of Lecture Notes in Computer Science, pages 93-116. Springer Berlin Heidelberg, 2010.

[65] C. Runciman, M. Naylor, and F. Lindblad. SmallCheck and Lazy SmallCheck: automatic exhaustive testing for small values In 1 st ACM SIGPLAN Symposium on Haskell. 2008.

[66] E. L. Seidel, N. Vazou, and R. Jhala. Type targeted testing In Programming Languages and Systems - 24th European Symposium on Programming, ESOP 2015, Held as Part of the European Joint Conferences on Theory and Practice of Software, ETAPS 2015, London, UK, April 11-18, 2015. Proceedings, 2015.

[67] K. Sen, D. Marinov, and G. Agha. CUTE: a concolic unit testing engine for C In 10th European software engineering conference held jointly with 13th ACM SIGSOFT international symposium on Foundations of software engineering. 2005.

[68] P. Tarau. On type-directed generation of lambda terms In Proceedings of the Technical Communications of the 31 st International Conference on Logic Programming (ICLP 2015), Cork, Ireland, August 31 September 4, 2015., 2015.

[69] A. P. Tolmach and S. Antoy. A monadic semantics for core Curry Electr. Notes Theor. Comput. Sci., 86(3):16-34, 2003.

[70] E. Torlak and R. Bodík. A lightweight symbolic virtual machine for solver-aided host languages In ACM SIGPLAN Conference on Programming Language Design and Implementation. 2014. 